\title{
Panel Unit Root Tests in the Presence of a Multifactor Error Structure
}

\author{
M. Hashem Pesaran, L. Vanessa Smith and Takashi \\ Yamagata
}

December 2007

CWPE 0775

Paper presented at the conference on "Institutions, Public Policy and Economic Outcomes" held in August 2007 and sponsored by the Economic and Social Research Council (grant no.RES 000-22-1743). 


\title{
Panel Unit Root Tests in the Presence of a Multifactor Error Structure*
}

\author{
M. Hashem Pesaran ${ }^{a}$ \\ L. Vanessa $\operatorname{Smith}^{b}$ \\ Takashi Yamagata ${ }^{c}$ \\ ${ }^{a}$ Centre for International Macroeconomics and Finance (CIMF), University of Cambridge, and USC \\ ${ }^{b}$ Centre for Financial Analysis and Policy (CFAP), University of Cambridge \\ ${ }^{c}$ University of York
}

December 2007

\begin{abstract}
This paper extends the cross sectionally augmented panel unit root test proposed by Pesaran (2007) to the case of a multifactor error structure. The basic idea is to exploit information regarding the unobserved factors that are shared by other time series in addition to the variable under consideration. Importantly, our test procedure only requires specification of the maximum number of factors, in contrast to other panel unit root tests based on principal components that require in addition the estimation of the number of factors as well as the factors themselves. Small sample properties of the proposed test are investigated by Monte Carlo experiments, which suggest that it controls well for size in almost all cases, especially in the presence of serial correlation in the error term, contrary to alternative test statistics. Empirical applications to Fisher's inflation parity and real equity prices across different markets illustrate how the proposed test works in practice.
\end{abstract}

JEL-Classification: C12, C15, C22, C23

Keywords: Panel unit root tests, Cross Section Dependence, Multi-factor Residual Structure, Fisher Inflation Parity, Real Equity Prices.

${ }^{*}$ We would like to thank Serena Ng for sharing her codes with us for the calculation of the Bai and Ng (2007) statistics, Joachim Westerlund for useful comments on an earlier version of the paper, as well as participants at the conference in honour of Paul Newbold at the Univeristy of Nottingham, November 2007. 


\section{Introduction}

There is now a sizeable literature on testing for unit roots in panels where both cross section $(N)$ and time $(T)$ dimensions are relatively large. Reviews of this literature are provided in Banerjee (1999), Baltagi and Kao (2000), Choi (2004), and more recently in Breitung and Pesaran (2007). The so called first generation panel unit root tests pioneered by Levin, Lin and Chu (2002) and Im, Pesaran and Shin (2003) focussed on panels where the idiosyncratic errors were cross sectionally uncorrelated. More recently, to deal with a number of applications such as testing for purchasing power parity or output convergence, the interest has shifted to the case where the errors are allowed to be cross sectionally correlated using a residual factor structure. ${ }^{1}$ These second generation tests include the contributions of Moon and Perron (2004), Bai and Ng $(2004,2007)$ and Pesaran $(2007) .{ }^{2}$ The tests proposed by Moon and Perron (2004) and Pesaran (2007) assume that under the null of unit roots the common factor components have the same order of integration as the idiosyncratic components, whilst the test procedures of Bai and $\mathrm{Ng}(2004,2007)$ allow the order of integration of the factors to differ from that of the idiosyncratic components, by assuming different processes generating the two. A small sample comparison of some of these tests is provided in Gengenbach, Palm and Urbain (2006).

In the panel unit root test proposed by Pesaran (2007) cross section dependence is accounted for by augmenting the individual ADF regressions of $y_{i t}$ with cross section averages of the dependent variable (current and lagged values, $\Delta \bar{y}_{t}, \bar{y}_{t-1}=n^{-1} \sum_{j=1}^{N} y_{j, t-1}$ ). These cross section averages are used as proxies for the assumed single unobserved common factor. The panel test statistic is then based on the average of the individual t-statistics over the cross section units and is shown to be free of nuisance parameters, although it has a non-normal limit distribution as $N$ and $T \rightarrow \infty$. However, the validity of Pesaran's test for a single unobserved common factor could be a limitation in practice. Bai and $\mathrm{Ng}(2004,2007)$ consider whether the source of nonstationarity is due to the common factor and/or idiosyncratic component. Their method involves applying unit root tests to the common factors and the idiosyncratic component separately, where the unobserved factors are replaced with consistent estimates obtained by use of principal components (PC). The pooled tests they propose require an estimate of the true number of factors and the factors themselves. Moon and Perron (2004) follow a similar approach in that they base their test on a principal components estimator of common factors. In particular, their test is based on de-factored observations obtained by projecting the panel data onto the space orthogonal to the (estimated) factor loadings.

In this paper we extend the test of Pesaran (2007) and propose a simple panel unit root test that is valid in the more general case of multiple common factors. In so doing we utilise the information contained in a number of $k$ additional variables, $\mathbf{x}_{i t}$, that are assumed to share the same common factors as the original series of interest, $y_{i t}$. The ADF regression for $y_{i t}$ is then augmented by the cross section averages of the dependent variable as well as the additional regressors.

We find that the limit distribution of our proposed unit root test does not depend on any nuisance parameters such as the factor loadings or cross section heteroskedasticity, so long as

\footnotetext{
${ }^{1}$ It is also possible to allow for cross sectionally correlated errors by adjusting the standard errors of the pooled estimate of the autoregressive coefficient. Breitung and Das (2007), however, show that validity of these tests depend on whether the factors and/or the errors are non-stationary.

${ }^{2}$ Other panel unit root tests include that of Chang (2002) that employs a non-linear IV method to account for cross-section correlation and Phillips and Sul (2003) who use an orthogonalisation procedure. The former is valid for a fixed $N$ and large $T$.
} 
$k+1$ is greater or equal to the true number of factors, $m$. Therefore, the distribution of the test statistic can be well approximated by the distribution based on the augmented regression with $k$ additional regressors for $k+1 \geq m$. This means that our approach does not require the knowledge of the true number of factors, in contrast to the panel unit root tests based on principal components that require, in addition to the specification of the maximum number of factors, the estimation of the number of factors and the factors themselves.

Using Monte Carlo techniques, we show that our proposed test has the correct size in almost all experiments, especially in the presence of serial correlation in the errors terms, contrary to the tests of Bai and $\mathrm{Ng}$ (2007) and Moon and Perron (2004). ${ }^{3}$ In terms of power, our test tends to display higher power as compared to the other tests for large $T$ and $N$, both when the ADF regressions contain an intercept only and/or a linear trend. As shown in Moon, Perron and Phillips (2006) the power of panel unit root tests in the presence of linear trends could be quite low.

The plan of the paper is as follows. Section 2 presents the panel data model and the testing procedure and derives the asymptotic distribution of the proposed cross sectionally augmented panel unit root test. Section 3 shows how the critical values of the test are obtained. The small sample performance of the proposed test, as compared to other tests proposed in the literature, is investigated in Section 4. Section 5 illustrates its use in an empirical application. Section 6 provides some concluding remarks.

Notation: $K$ denotes a finite positive constant, $\|\mathbf{A}\|=\left[\operatorname{tr}\left(\mathbf{A A}^{\prime}\right)\right]^{1 / 2}, \mathbf{A}^{-}$denotes the generalised inverse of $\mathbf{A}, \mathbf{I}_{q}$ is a $q \times q$ identity matrix, $\boldsymbol{\tau}_{q}$ and $\mathbf{0}_{q}$ are $(q \times 1)$ vectors of ones and zeros, respectively, $\mathbf{0}_{q \times r}$ is a $(q \times r)$ null matrix, $\stackrel{N}{\Longrightarrow}(\stackrel{N}{\longrightarrow})$ denotes convergence in distribution (quatradic mean (q.m.) or mean square errors) with $T$ fixed as $N \rightarrow \infty, \stackrel{T}{\Longrightarrow}(\stackrel{T}{\rightarrow})$ denotes convergence in distribution (q.m.) with $N$ fixed (or when there is no $N$-dependence) as $T \rightarrow \infty$, $\stackrel{N, T}{\Longrightarrow}$ denotes sequential convergence with $N \rightarrow \infty$ first followed by $T \rightarrow \infty, \stackrel{(N, T)_{j}}{\Longrightarrow}$ denotes joint convergence with $N, T \rightarrow \infty$ jointly with some restriction on the expansion rate of $T / N$, if any.

\section{Panel Data Model and Tests}

Let $y_{i t}$ be the observation on the $i^{\text {th }}$ cross section unit at time $t$ generated as

$$
\Delta y_{i t}=\beta_{i}\left(y_{i, t-1}-\boldsymbol{\alpha}_{i}^{\prime} \mathbf{d}_{t-1}\right)+\boldsymbol{\alpha}_{i}^{\prime} \Delta \mathbf{d}_{t}+u_{i t}, i=1,2, \ldots, N ; t=1,2, \ldots, T
$$

where $\beta_{i}=-\left(1-\rho_{i}\right), \mathbf{d}_{t}$ is $2 \times 1$ vector of observed common effects including an intercept and a linear trend so that $\mathbf{d}_{t}=(1, t)^{\prime}$. Consider the following multifactor error structure

$$
u_{i t}=\gamma_{i}^{\prime} \mathbf{f}_{t}+\varepsilon_{i t}
$$

where $\mathbf{f}_{t}$ is an $m \times 1$ vector of unobserved common effects, $\gamma_{i}$ is the associated vector of factor loadings, and $\varepsilon_{i t}$ is the idiosyncratic component. This set up generalises Pesaran's (2007) one factor error specification. We assume that these error processes satisfy the following assumptions:

\footnotetext{
${ }^{3}$ As pointed out recently by Westerlund and Larsson (2007), the test proposed by Bai and Ng (2004) which is based on the pooled p-values of individual panel t-statistics, is asymptotically invalid owing to an asymptotic bias that arises when replacing the unobserved idiosyncratic components by their estimates. For this reason we do not consider this test in the Monte Carlo experiments that follow.
} 
Assumption 1: The idiosyncratic shocks, $\varepsilon_{i t}, i=1,2, \ldots, N ; t=1,2, \ldots, T$, are independently distributed both across $i$ and $t$, have zero means, variances $0<\sigma_{i}^{2}<K<\infty$ and finite fourth-order moments. This assumption, which implies that the idiosyncratic shocks are non-autocorrelated, will be relaxed in Section 2.1.

Assumption 2: The $m \times 1$ vector $\mathbf{f}_{t}$ is a covariance stationary process, with absolute summable autocovariances, distributed independently of $\varepsilon_{i t^{\prime}}$ for all $i, t$ and $t^{\prime}$. Specifically, we assume that $\mathbf{f}_{t}=\mathbf{\Psi}(L) \mathbf{e}_{t}$ where the error terms $\mathbf{e}_{t} \sim \operatorname{IID}(\mathbf{0}, \boldsymbol{\Omega})$, with finite fourth-order moments and a positive definite matrix $\boldsymbol{\Omega}$, and $\boldsymbol{\Psi}(L)=\sum_{\ell=0}^{\infty} \underset{m \times m}{\mathbf{\Psi}_{\ell}} L^{\ell}$ where $\left\{\ell \mathbf{\Psi}_{\ell}\right\}_{\ell=0}^{\infty}$ are absolute summable so that $\operatorname{Var}\left(\mathbf{f}_{t}\right)$ is bounded and positive definite and $[\boldsymbol{\Psi}(L)]^{-1}$ exists. In particular, $\boldsymbol{\Sigma}_{f s}=E\left(\mathbf{f}_{t} \mathbf{f}_{t-s}\right)=\sum_{\ell=0}^{\infty} \mathbf{\Psi}_{s+\ell} \boldsymbol{\Omega} \boldsymbol{\Psi}_{\ell}^{\prime} \leq \mathbf{K}<\infty$, for $s=0,1,2 \ldots$ where $\mathbf{K}$ is a fixed bounded matrix, such that $\|\mathbf{K}\|<K$. Further, $\boldsymbol{\Lambda}_{f}=\sum_{\ell=0}^{\infty} \boldsymbol{\Psi}_{\ell} \mathbf{P}$ where $\mathbf{P}$ is obtained as the Cholesky factorization of $\boldsymbol{\Omega}=\mathbf{P P}^{\prime}$.

Assumption 3: The unobserved factor loadings, $\gamma_{i}$ are bounded, $\left\|\gamma_{i}\right\|<K<\infty$, for all $i$. Combining (1) and (2) it follows that

$$
\Delta y_{i t}=\beta_{i}\left(y_{i, t-1}-\boldsymbol{\alpha}_{i}^{\prime} \mathbf{d}_{t-1}\right)+\boldsymbol{\alpha}_{i}^{\prime} \Delta \mathbf{d}_{t}+\gamma_{i}^{\prime} \mathbf{f}_{t}+\varepsilon_{i t} .
$$

The hypothesis that all the series, $y_{i t}$, have a unit root and not cointegrated can be expressed as

$$
H_{0}: \beta_{i}=0 \text { for all } i
$$

against the alternative

$$
H_{1}: \beta_{i}<0 \text { for } i=1,2, \ldots, N_{1}, \beta_{i}=0 \text { for } i=N_{1}+1, N_{1}+2, . ., N
$$

where $N_{1} / N \rightarrow \kappa$ and $0<\kappa \leq 1$ as $N \rightarrow \infty$.

Note that under the null hypothesis, (3) can be solved for $y_{i t}$ to yield

$$
y_{i t}=\tilde{y}_{i 0}+\boldsymbol{\alpha}_{i}^{\prime} \mathbf{d}_{t}+\gamma_{i}^{\prime} \mathbf{s}_{f t}+s_{i t},
$$

where

$$
\begin{aligned}
\mathbf{s}_{f t} & =\mathbf{f}_{1}+\mathbf{f}_{2}+\ldots+\mathbf{f}_{t}, \\
s_{i t} & =\varepsilon_{1 t}+\varepsilon_{2 t}+\ldots+\varepsilon_{i t},
\end{aligned}
$$

and $\tilde{y}_{i 0}=y_{i 0}-\boldsymbol{\alpha}_{i}^{\prime} \mathbf{d}_{0}$. Therefore, under $H_{0}$ and Assumptions 1 and $3, y_{i t}$ is composed of a determinstic component, $\tilde{y}_{i 0}+\boldsymbol{\alpha}_{i}^{\prime} \mathbf{d}_{t}$, a common stochastic component, $\mathbf{s}_{f t} \sim I(1)$, and an idiosyncratic component, $s_{i t} \sim I(1)$, so that while all units of the panel share the common stochastic trends, $\mathbf{s}_{f t}$, there is no cointegration among them. Under the alternative hypothesis, $\beta_{i}<0$, we have $y_{i t} \sim I(0)$, and it is essential that $\mathbf{f}_{t}$ is at most an $I(0)$ process.

In the case where $m=1$, Pesaran (2007) proposes a test of $\beta_{i}=0$ jointly with $f_{t} \sim I(0)$, based on DF (or ADF) regressions augmented by the current and lagged cross section averages of $y_{i t}$ as proxies for the unobserved $f_{t}$. He shows that the resultant test is asymptotically invariant to the factor loadings, $\gamma_{i}$. To extend Pesaran's approach here we assume that in addition to $y_{i t}$, there exist other observables, say $\mathbf{x}_{i t}$, that are driven by at least the same set of common trends, $\mathbf{s}_{f t}$ that drive $y_{i t}$. For example, in the analysis of output convergence it is reasonable to argue that output, investment, consumption, real equity prices, and oil prices have the same set of factors in common. Similarly, short term and long terms interest rates and inflation 
across countries are likely to have a number of factors in common. In practice these factors can be either used directly or one could use the cumulated principle components of their first differences.

More specifically, suppose the $k \times 1$ vector of additional regressors follow the general linear process

$$
\mathbf{x}_{i t}=\tilde{\mathbf{x}}_{i 0}+\boldsymbol{\Gamma}_{i x} \mathbf{s}_{f t}+\mathbf{A}_{i x} \mathbf{d}_{t}+\mathbf{v}_{i x t},
$$

where $\mathbf{x}_{i t}=\left(x_{i 1 t}, x_{i 2 t}, \ldots, x_{i k t}\right)^{\prime}, \boldsymbol{\Gamma}_{i x}=\left(\boldsymbol{\gamma}_{i 1}, \boldsymbol{\gamma}_{i 2}, \ldots, \boldsymbol{\gamma}_{i k}\right)^{\prime}, \mathbf{A}_{i x}=\left(\mathbf{a}_{i 1}, \mathbf{a}_{i 2}, \ldots, \mathbf{a}_{i k}\right)^{\prime}, \tilde{\mathbf{x}}_{i 0}=\mathbf{x}_{i 0}-$ $\mathbf{A}_{i x} \mathbf{d}_{0}$ and $\mathbf{v}_{i x t}$ is the idiosyncratic component of $\mathbf{x}_{i t}$ that could be either $I(1)$ or $I(0)$. Here we assume $\mathbf{v}_{i x t}$ to be $I(1)$, which rules out cointegration among the $\mathbf{x}_{i t}^{\prime} s$, and we consider $\Delta \mathbf{v}_{i x t}$ to follow a stationary linear process. We also assume that the $k \times 1$ vector $\mathbf{v}_{\text {ixt }}$ is distributed independently of $\varepsilon_{i t^{\prime}}$ for all $i, t$ and $t^{\prime}$.

Combining (6) and (7) we have

$$
\mathbf{z}_{i t}=\tilde{\mathbf{z}}_{i 0}+\boldsymbol{\Gamma}_{i} \mathbf{s}_{f t}+\mathbf{A}_{i} \mathbf{d}_{t}+\mathbf{v}_{i t},
$$

where $\mathbf{z}_{i t}=\left(y_{i t}, \mathbf{x}_{i t}^{\prime}\right)^{\prime}, \tilde{\mathbf{z}}_{i 0}=\left(\tilde{y}_{i 0}, \tilde{\mathbf{x}}_{i 0}^{\prime}\right)^{\prime}, \boldsymbol{\Gamma}_{i}=\left(\boldsymbol{\gamma}_{i}, \boldsymbol{\Gamma}_{i x}^{\prime}\right)^{\prime}, \mathbf{A}_{i}=\left(\boldsymbol{\alpha}_{i}, \mathbf{A}_{i x}^{\prime}\right)^{\prime}, \mathbf{v}_{i t}=\left(s_{i t}, \mathbf{v}_{i x t}^{\prime}\right)^{\prime}$.

Assumption 4: The $(k+1) \times m$ matrix of factor loadings $\boldsymbol{\Gamma}_{i}$ are such that

$$
\operatorname{rank}[\bar{\Gamma}]=m \leq k+1, \text { for any } N,
$$

where $\bar{\Gamma}=N^{-1} \sum_{i=1}^{N} \boldsymbol{\Gamma}_{i}$, and $\bar{\Gamma} \stackrel{N}{\rightarrow} \boldsymbol{\Gamma}$, where $\boldsymbol{\Gamma}$ is a fixed bounded matrix with rank $m$.

Assumption 5: $E|| \mathbf{f}_{0}|| \leq K$, and $E|| \tilde{\mathbf{z}}_{i 0}|| \leq K, E\left|\mathbf{v}_{i x 0}\right| \leq K$, and $E\left|\varepsilon_{i 0}\right| \leq K$ for all $i$.

Averaging (8) over $i$ now yields

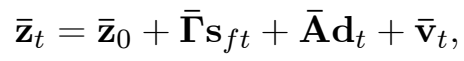

where $\overline{\mathbf{z}}_{t}=N^{-1} \sum_{i=1}^{N} \mathbf{z}_{i t}, \overline{\boldsymbol{\Gamma}}=N^{-1} \sum_{i=1}^{N} \boldsymbol{\Gamma}_{i}, \overline{\mathbf{A}}=N^{-1} \sum_{i=1}^{N} \mathbf{A}_{i}$, and $\overline{\mathbf{v}}_{t}=N^{-1} \sum_{i=1}^{N} \mathbf{v}_{i t}{ }^{4}$ Writing (3), (8) and (10) in matrix notation, under the null for each $i$ we have

$$
\begin{gathered}
\Delta \mathbf{y}_{i}=\mathbf{F} \boldsymbol{\gamma}_{i}+\Delta \mathbf{D} \boldsymbol{\alpha}_{i}+\varepsilon_{i}, \\
\Delta \mathbf{Z}_{i}=\mathbf{F} \boldsymbol{\Gamma}_{i}^{\prime}+\Delta \mathbf{D} \mathbf{A}_{i}^{\prime}+\Delta \mathbf{V}_{i}, \\
\Delta \overline{\mathbf{Z}}=\mathbf{F} \overline{\boldsymbol{\Gamma}}^{\prime}+\Delta \mathbf{D} \overline{\mathbf{A}}^{\prime}+\Delta \overline{\mathbf{V}}
\end{gathered}
$$

where $\mathbf{F}=\left(\mathbf{f}_{1}, \mathbf{f}_{2}, \ldots, \mathbf{f}_{T}\right)^{\prime}$ with $\mathbf{f}_{0}=\mathbf{0}_{m}, \Delta \mathbf{D}=\left(\Delta \mathbf{d}_{1}, \Delta \mathbf{d}_{2}, \ldots, \Delta \mathbf{d}_{T}\right)^{\prime}, \boldsymbol{\varepsilon}_{i}=\left(\varepsilon_{i 1}, \varepsilon_{i 2}, \ldots, \varepsilon_{i T}\right)^{\prime}$ with $\varepsilon_{i 0}=0, \Delta \mathbf{Z}_{i}=\left(\Delta \mathbf{z}_{i 1}, \Delta \mathbf{z}_{i 2}, \ldots, \Delta \mathbf{z}_{i T}\right)^{\prime}, \Delta \mathbf{V}_{i}=\left(\Delta \mathbf{v}_{i 1}, \Delta \mathbf{v}_{i 2}, \ldots, \Delta \mathbf{v}_{i T}\right)^{\prime}, \Delta \overline{\mathbf{Z}}=\left(\Delta \overline{\mathbf{z}}_{i 1}, \Delta \overline{\mathbf{z}}_{i 2}, \ldots, \Delta \overline{\mathbf{z}}_{i T}\right)^{\prime}$ and $\Delta \overline{\mathbf{V}}=\left(\Delta \overline{\mathbf{v}}_{1}, \Delta \overline{\mathbf{v}}_{2}, \ldots, \Delta \overline{\mathbf{v}}_{T}\right)^{\prime}$. From (13) under rank condition (9) it follows that

$$
\mathbf{F}=\left[\Delta \overline{\mathbf{Z}}-\Delta \mathbf{D} \overline{\mathbf{A}}^{\prime}-\Delta \overline{\mathbf{V}}\right] \overline{\mathbf{\Gamma}}\left(\overline{\boldsymbol{\Gamma}}^{\prime} \overline{\mathbf{\Gamma}}\right)^{-1}
$$

However, from A.2.1 in Appendix A we have that $\Delta \overline{\mathbf{V}} \stackrel{N}{\rightarrow} \mathbf{0}$ as $N \rightarrow \infty$ for each $t$ and hence we obtain that

$$
\mathbf{F}-\left[\Delta \overline{\mathbf{Z}}-\Delta \mathbf{D} \overline{\mathbf{A}}^{\prime}-\Delta \overline{\mathbf{V}}\right] \overline{\boldsymbol{\Gamma}}\left(\overline{\boldsymbol{\Gamma}}^{\prime} \overline{\boldsymbol{\Gamma}}\right)^{-1} \stackrel{N}{\rightarrow} \mathbf{0}, \text { as } N \rightarrow \infty .
$$

\footnotetext{
${ }^{4}$ Weighted cross section averages could also be used as in Pesaran (2007) with appropriate granularity restrictions on the weights.
} 
In view of the above we shall base our test of the panel unit root on the $t$-ratio of the ordinary least square (OLS) estimate of $b_{i}\left(\hat{b}_{i}\right)$ in the following cross sectionally augmented regression

$$
\Delta y_{i t}=b_{i} y_{i t-1}+\mathbf{c}_{i}^{\prime} \overline{\mathbf{z}}_{t-1}+\mathbf{h}_{i}^{\prime} \Delta \overline{\mathbf{z}}_{t}+\mathbf{g}_{i}^{\prime} \Delta \mathbf{d}_{t}+\epsilon_{i t} .
$$

The $t$-ratio of $\hat{b}_{i}$ in this regression is given by

$$
t_{i}(N, T)=\frac{\Delta \mathbf{y}_{i}^{\prime} \overline{\mathbf{M}} \mathbf{y}_{i,-1}}{\hat{\sigma}_{i}\left(\mathbf{y}_{i,-1}^{\prime} \overline{\mathbf{M}} \mathbf{y}_{i,-1}\right)^{1 / 2}}=\frac{\sqrt{T-(2 k+4)} \Delta \mathbf{y}_{i}^{\prime} \overline{\mathbf{M}} \mathbf{y}_{i,-1}}{\left(\Delta \mathbf{y}_{i}^{\prime} \overline{\mathbf{M}}_{i} \Delta \mathbf{y}_{i}\right)^{1 / 2}\left(\mathbf{y}_{i,-1}^{\prime} \overline{\mathbf{M}} \mathbf{y}_{i,-1}\right)^{1 / 2}},
$$

where $\Delta \mathbf{y}_{i}=\left(\Delta y_{i 1}, \Delta y_{i 2}, \ldots, \Delta y_{i T}\right)^{\prime}, \mathbf{y}_{i,-1}=\left(y_{i 0}, y_{i 1}, \ldots, y_{i, T-1}\right)^{\prime}, \overline{\mathbf{M}}=\mathbf{I}_{T}-\overline{\mathbf{W}}\left(\overline{\mathbf{W}}^{\prime} \overline{\mathbf{W}}\right)^{-1} \overline{\mathbf{W}}^{\prime}$, $\overline{\mathbf{W}}=\left(\overline{\mathbf{w}}_{1}, \overline{\mathbf{w}}_{2}, \ldots, \overline{\mathbf{w}}_{T}\right)^{\prime}, \overline{\mathbf{w}}_{t}=\left(\Delta \overline{\mathbf{z}}_{t}^{\prime}, \mathbf{d}_{t}^{\prime}, \overline{\mathbf{z}}_{t-1}^{\prime}\right)^{\prime}$,

$$
\hat{\sigma}_{i}^{2}=\frac{\Delta \mathbf{y}_{i}^{\prime} \overline{\mathbf{M}}_{i} \Delta \mathbf{y}_{i}}{T-(2 k+4)}
$$

and $\overline{\mathbf{M}}_{i}=\mathbf{I}_{T}-\overline{\mathbf{W}}_{i}\left(\overline{\mathbf{W}}_{i}^{\prime} \overline{\mathbf{W}}_{i}\right)^{-1} \overline{\mathbf{W}}_{i}^{\prime}$, with $\overline{\mathbf{W}}_{i}=\left(\overline{\mathbf{W}}, \mathbf{y}_{i,-1}\right)$.

Using (14) in (11)

$$
\Delta \mathbf{y}_{i}=\Delta \overline{\mathbf{Z}} \boldsymbol{\delta}_{i}+\Delta \mathbf{D} \boldsymbol{\alpha}_{i}^{*}+\sigma_{i} \boldsymbol{v}_{i}
$$

where

$$
\begin{gathered}
\boldsymbol{\delta}_{i}=\overline{\boldsymbol{\Gamma}}\left(\overline{\boldsymbol{\Gamma}}^{\prime} \overline{\boldsymbol{\Gamma}}\right)^{-1} \boldsymbol{\gamma}_{i}, \boldsymbol{\alpha}_{i}^{*}=\boldsymbol{\alpha}_{i}-\overline{\mathbf{A}}^{\prime} \boldsymbol{\delta}_{i}, \\
\boldsymbol{v}_{i}=\boldsymbol{\xi}_{i} / \sigma_{i},
\end{gathered}
$$

with $\boldsymbol{\xi}_{i}=\left(\varepsilon_{i}-\Delta \overline{\mathbf{V}} \boldsymbol{\delta}_{i}\right), \boldsymbol{\xi}_{i}=\left(\xi_{i 1}, \xi_{i 2}, \ldots, \xi_{i T}\right)^{\prime}$ and $E\left(\boldsymbol{\xi}_{i} \boldsymbol{\xi}_{i}^{\prime}\right)=\sigma_{i}^{2} \mathbf{I}_{T}+O\left(N^{-1}\right)$. Therefore, we have

$$
\overline{\mathbf{M}} \Delta \mathbf{y}_{i}=\sigma_{i} \overline{\mathbf{M}} \boldsymbol{v}_{i} .
$$

From (12) and (13) we obtain

$$
\mathbf{Z}_{i,-1}=\boldsymbol{\tau}_{T} \otimes \tilde{\mathbf{z}}_{i 0}^{\prime}+\mathbf{S}_{f,-1} \boldsymbol{\Gamma}_{i}^{\prime}+\mathbf{D}_{-1} \mathbf{A}_{i}^{\prime}+\mathbf{V}_{i,-1} .
$$

which expressing $\mathbf{Z}_{i,-1}$ as deviation from its initial value. Also

$$
\overline{\mathbf{Z}}_{-1}=\boldsymbol{\tau}_{T} \otimes \overline{\mathbf{z}}_{0}^{\prime}+\mathbf{S}_{f,-1} \overline{\boldsymbol{\Gamma}}^{\prime}+\mathbf{D}_{-1} \overline{\mathbf{A}}^{\prime}+\mathbf{V}_{i,-1}
$$

where $\mathbf{S}_{f,-1}=\left(\mathbf{0}_{m}, \mathbf{s}_{f 1}, \ldots, \mathbf{s}_{f, T-1}\right)^{\prime}, \mathbf{D}_{-1}=\left(\mathbf{d}_{0}, \mathbf{d}_{1}, \ldots, \mathbf{d}_{T-1}\right)^{\prime}$, with $\mathbf{d}_{0}=(1,0)^{\prime}, \mathbf{Z}_{i,-1}=$ $\left(\tilde{\mathbf{z}}_{i 0}, \mathbf{z}_{i 1}, \ldots, \mathbf{z}_{i T-1}\right)^{\prime}, \mathbf{V}_{i,-1}=\left(\mathbf{v}_{i 0}, \mathbf{v}_{i 1}, \ldots, \mathbf{v}_{i, T-1}\right)^{\prime}, \overline{\mathbf{Z}}_{-1}=\left(\overline{\mathbf{z}}_{0}, \overline{\mathbf{z}}_{1}, \ldots, \overline{\mathbf{z}}_{T-1}\right)^{\prime}$ and $\overline{\mathbf{V}}_{-1}=\left(\overline{\mathbf{v}}_{0}, \overline{\mathbf{v}}_{1}, \ldots, \overline{\mathbf{v}}_{T-1}\right)^{\prime}$.

Similarly from (18)

$$
\mathbf{y}_{i,-1}=\stackrel{\circ}{y}_{i 0} \boldsymbol{\tau}_{T}+\overline{\mathbf{Z}}_{-1} \boldsymbol{\delta}_{i}+\mathbf{D}_{-1} \boldsymbol{\alpha}_{i}^{*}+\sigma_{i} \stackrel{\circ}{\mathfrak{s}}_{i,-1}
$$

where

$$
\stackrel{\mathfrak{s}}{i,-1}=\left(\mathbf{s}_{i,-1}-\overline{\mathbf{V}}_{-1} \boldsymbol{\delta}_{i}\right) / \sigma_{i},
$$

$\mathbf{s}_{i,-1}=\left(0, s_{i 1}, \ldots, s_{i, T-1}\right)^{\prime}$ and $\stackrel{\circ}{y 0}_{i 0}=y_{i 0}-\boldsymbol{\delta}_{i}^{\prime}\left(\overline{\mathbf{z}}_{0}+\overline{\mathbf{v}}_{0}\right)-\boldsymbol{\alpha}_{i}^{* \prime} \mathbf{d}_{0}$.

Therefore,

$$
\overline{\mathbf{M}} \mathbf{y}_{i,-1}=\sigma_{i} \overline{\mathbf{M}}_{\mathfrak{s}_{i,-1}}
$$


Using (21) and (26), $t_{i}(N, T)$ can be re-written as

$$
t_{i}(N, T)=\frac{\boldsymbol{v}_{i}^{\prime} \overline{\mathbf{M}}_{\mathfrak{s}_{i,-1}}}{\left(\frac{\boldsymbol{v}_{i}^{\prime} \overline{\mathbf{M}}_{i} \boldsymbol{v}_{i}}{T-2 k-4}\right)^{1 / 2}\left(\stackrel{\mathfrak{s}}{i,-1}_{i, 1}^{\prime} \overline{\mathbf{M}}_{\mathfrak{s}_{i,-1}}\right)^{1 / 2}} .
$$

For fixed $N$ and $T$, the distribution of $t_{i}(N, T)$ will depend on the nuisance parameters through their effects on $\overline{\mathbf{M}}_{i}$ and $\overline{\mathbf{M}}$. However, this dependence vanishes as $N \rightarrow \infty$, for a fixed $T$. In the case of fixed $T$ however, the effect of the initial cross section mean, $\overline{\mathbf{z}}_{0}$, must be eliminated in order to ensure that $t_{i}(N, T)$ does not depend on nuisance parameters. This can be achieved by applying the test to the deviation $\mathbf{z}_{i t}-\overline{\mathbf{z}}_{0}$.

Before proceeding further, for notational convenience, we set

$$
\begin{aligned}
\mathbf{S}_{\mathbf{v} x i,-1} & =\mathbf{V}_{x i,-1}, \text { with } \mathbf{S}_{\mathbf{v} x i,-1}=\left(\mathbf{0}_{k}, \mathbf{s}_{v x i 1}, \ldots, \mathbf{s}_{v x i, T-1}\right)^{\prime} \\
\mathbf{S}_{\mathbf{v} i,-1} & =\mathbf{V}_{i,-1} \text { so that } \mathbf{S}_{\mathbf{v} i,-1}=\left(\mathbf{s}_{i,-1}, \mathbf{S}_{\mathbf{v} x i,-1}\right)
\end{aligned}
$$

Also

$$
\overline{\mathbf{s}}_{-1}=N^{-1} \sum_{i=1}^{N} \mathbf{s}_{i,-1}, \overline{\mathbf{S}}_{\mathbf{v} x,-1}=N^{-1} \sum_{i=1}^{N} \mathbf{S}_{\mathbf{v} x i,-1}, \overline{\mathbf{S}}_{\mathbf{v},-1}=\left(\overline{\mathbf{s}}_{-1}, \overline{\mathbf{S}}_{\mathbf{v} x,-1}\right)
$$

and so (25) becomes $\stackrel{\circ}{\mathfrak{s}}_{i,-1}=\left[\mathbf{s}_{i,-1}-\overline{\mathbf{S}}_{\mathbf{v},-1} \boldsymbol{\delta}_{i}\right] / \sigma_{i}$.

In the theorem that follows we derive the asymptotic distribution of the $t_{i}(N, T)$ statistic under the null hypothesis. Note that all order results and proofs of theorems given in the Appendix are derived for the case where $\mathbf{d}_{t}=1, t=0,1, \ldots, T$, which implies $\Delta \mathbf{D}=\mathbf{0}$ and $\mathbf{D}_{-1}-\boldsymbol{\tau}_{T} \otimes \mathbf{d}_{0}^{\prime}=\mathbf{0}$. The asymptotic results for the case where $\mathbf{d}_{t}=(1, t)^{\prime}$ can be derived in a similar manner.

Theorem 2.1 Suppose the series $\mathbf{z}_{i t}$, for $i=1,2, \ldots, N$, and $t=1,2, \ldots, T$, are generated under (4) according to (8), $\mathbf{d}_{s}=1, s=0,1, \ldots, T$, with $\overline{\mathbf{z}}_{i 0}$ set to a zero vector. Then under Assumptions 1-5, the distribution of $t_{i}(N, T)$ given by (27), will be free of nuisance parameters as $N \rightarrow \infty$ for any fixed $T>2 k+4$. In particular, we have (in quadratic mean)

$$
t_{i}(N, T) \stackrel{N}{\rightarrow} \frac{\frac{\boldsymbol{\varepsilon}_{i}^{\prime} \mathbf{s}_{i,-1}}{\sigma_{i}^{2} T}-\mathbf{q}_{i T}^{\prime} \mathbf{\Upsilon}_{f T}^{-1} \mathbf{h}_{i T}}{\left(\frac{\boldsymbol{\varepsilon}_{i}^{\prime} \boldsymbol{\varepsilon}_{i}}{\sigma_{i}^{2}(T-2 k-4)}-\frac{\mathbf{g}_{i T}^{\prime} \mathbf{Q}_{i T}^{-1} \mathbf{g}_{i T}}{(T-2 k-4)}\right)^{1 / 2}\left(\frac{\mathbf{s}_{i,-1}^{\prime} \mathbf{s}_{i,-1}}{\sigma_{i}^{2} T^{2}}-\mathbf{h}_{i T}^{\prime} \mathbf{\Upsilon}_{f T}^{-1} \mathbf{h}_{i T}\right)^{1 / 2}},
$$

where

$$
\begin{gathered}
\underset{(2 m+1 \times 1)}{\mathbf{q}_{i T}}=\left(\begin{array}{c}
\frac{\mathbf{F}^{\prime} \varepsilon_{i}}{\sigma_{i} \sqrt{T}} \\
\frac{\boldsymbol{\tau}_{T}^{\prime} \varepsilon_{i}}{\sigma_{i} \sqrt{T}} \\
\frac{\mathbf{S}_{f,-1}^{\prime} \varepsilon_{i}}{\sigma_{i} T}
\end{array}\right), \quad \underset{(2 m+1 \times 1)}{\mathbf{h}_{i T}}=\left(\begin{array}{c}
\frac{\mathbf{F}^{\prime} \mathbf{s}_{i,-1}}{\sigma_{i} T^{3 / 2}} \\
\frac{\boldsymbol{\tau}_{T}^{\prime} \mathbf{s}_{i,-1}}{\sigma_{i} T^{3 / 2}} \\
\frac{\mathbf{s}_{f,-1}^{\prime} \mathbf{s}_{i,-1}}{\sigma_{i} T^{2}}
\end{array}\right), \mathbf{g}_{i T}=\left(\begin{array}{c}
\mathbf{q}_{i T} \\
\frac{\mathbf{s}_{i,-1}^{\prime} \varepsilon_{i}}{\sigma_{i}^{2} T}
\end{array}\right) \\
\mathbf{\Upsilon}_{f T}=\left(\begin{array}{ccc}
\frac{\mathbf{F}^{\prime} \mathbf{F}}{T} & \frac{\mathbf{F}^{\prime} \boldsymbol{\tau}_{T}}{T} & \frac{\mathbf{F}^{\prime} \mathbf{S}_{f,-1}}{T^{3 / 2}} \\
\frac{\boldsymbol{\tau}_{T}^{\prime} \mathbf{F}}{T} & 1 & \frac{\boldsymbol{\tau}_{T}^{\prime} \mathbf{S}_{f,-1}}{\mathbf{S}_{f,-1}^{\prime} \mathbf{F}} \\
\frac{\mathbf{S}_{f,-1}^{\prime} \boldsymbol{\tau}_{T}}{T^{3 / 2}} & \frac{\mathbf{S}_{f,-1}^{\prime} \mathbf{S}_{f,-1}}{T^{3 / 2}}
\end{array}\right), \mathbf{Q}_{i T}=\left(\begin{array}{cc}
\mathbf{\Upsilon}_{f T} & \mathbf{h}_{i T} \\
\mathbf{h}_{i T}^{\prime} & \frac{\mathbf{s}_{i,-1}^{\prime} \mathbf{s}_{i,-1}}{\sigma_{i}^{2} T^{2}}
\end{array}\right) .
\end{gathered}
$$

See Appendix A.3 for a proof. 
Remark 2.1 The limit distribution of $t_{i}$ does not depend on the factor loadings and $\sigma_{i}$ but only on $\mathbf{f}_{t}$ and the standardised errors $\varepsilon_{i t} / \sigma_{i}$.

Theorem 2.2 Suppose the series $\mathbf{z}_{i t}$, for $i=1,2, \ldots, N, t=1,2, \ldots, T$, are generated under ( 4 ) according to (8) and $\mathbf{d}_{s}=1, s=0,1, \ldots, T$. Then under Assumptions 1-5 and as $N$ and $T \rightarrow \infty$, such that $\sqrt{T} / N \rightarrow 0, t_{i}(N, T)$ given by (27) has the same sequential $(N \rightarrow \infty, T \rightarrow \infty)$ and joint $\left[(N, T)_{j} \rightarrow \infty\right]$ limit distribution, is free of nuisance parameters and is given by

$$
C A D F_{i, f}=\frac{\int_{0}^{1} W_{i}(r) d W_{i}(r)-\boldsymbol{\omega}_{i f}^{\prime} \mathbf{G}_{f}^{-1} \boldsymbol{\pi}_{i f}}{\left(\int_{0}^{1} W_{i}^{2}(r) d r-\boldsymbol{\pi}_{i f}^{\prime} \mathbf{G}_{f}^{-1} \boldsymbol{\pi}_{i f}\right)^{1 / 2}},
$$

where

$$
\begin{gathered}
\boldsymbol{\omega}_{i f}=\left(\begin{array}{c}
W_{i}(1) \\
\int_{0}^{1}\left[\mathbf{W}_{f}(r)\right] d W_{i}(r)
\end{array}\right), \boldsymbol{\pi}_{i f}=\left(\begin{array}{c}
\int_{0}^{1} W_{i}(r) d r \\
\int_{0}^{1}\left[\mathbf{W}_{f}(r)\right] W_{i}(r)
\end{array}\right), \\
\mathbf{G}_{f}=\left(\begin{array}{c}
1 \\
\int_{0}^{1}\left[\mathbf{W}_{f}(r)\right] d r \quad \int_{0}^{1}\left[\mathbf{W}_{f}(r)\right]\left[\mathbf{W}_{f}(r)\right]^{\prime} d r
\end{array}\right) .
\end{gathered}
$$

See Appendix A.4 for a proof. Note that $C A D F_{i, f}$ does not depend on $\boldsymbol{\Lambda}_{f}$ as defined in Assumption 2.

Remark 2.2 $C A D F_{i, f}$ and $C A D F_{j, f}$ are dependently distributed with the same degree of dependence for all $i \neq j$.

Having established that the limit distribution of the individual $t_{i}(N, T)$ statistic is free of nuisance parameters, we now focus on panel unit root tests based on the average of these statistics defined by

$$
\operatorname{CIPS}(N, T)=N^{-1} \sum_{i=1}^{N} t_{i}(N, T) .
$$

As discussed in Pesaran (2007), in general it is difficult to establish moment conditions to prove

$$
N^{-1} \sum_{i=1}^{N}\left[t_{i}(N, T)-C A D F_{i}\right]=o_{p}(1)
$$

for $N$ and $T$ sufficiently large. To tackle this problem, following Pesaran (2007), we consider basing the test on a suitably truncted version of the $t_{i}(N, T)$ statistics, in such a way that

$$
t_{i}^{*}(N, T)= \begin{cases}t_{i}(N, T), & \text { if }-K_{1}<t_{i}(N, T)<K_{2}, \\ -K_{1}, & \text { if } t_{i}(N, T) \leq-K_{1}, \\ K_{2}, & \text { if } t_{i}(N, T) \geq K_{2},\end{cases}
$$


where $K_{1}$ and $K_{2}$ are positive constants that are sufficiently large so that $\operatorname{Pr}\left[-K_{1}<t_{i}(N, T)<\right.$ $\left.K_{2}\right]$ is sufficiently large. Using the normal approximation of $t_{i}(N, T)$, we would have $K_{1}=$ $-E\left(C A D F_{i}\right)-\Phi^{-1}(\varepsilon / 2) \sqrt{\operatorname{Var}\left(C A D F_{i}\right)}$, and $K_{2}=E\left(C A D F_{i}\right)+\Phi^{-1}(\varepsilon / 2) \sqrt{\operatorname{Var}\left(C A D F_{i}\right)}$, where $\Phi^{-1}($. ) is the inverse of the cumulative standard normal distribution function, and $\varepsilon$ is a sufficiently small positive constant. $K_{1}$ and $K_{2}$ can now be obtained using simulated values of $E\left(C A D F_{i}\right)$ and $\operatorname{Var}\left(C A D F_{i}\right)$ with $\varepsilon=1 \times 10^{-6}$ for $N=200$, and $T=200$.

The associated truncated panel unit root test is given by

$$
\operatorname{CIPS}^{*}(N, T)=N^{-1} \sum_{i=1}^{N} t_{i}^{*}(N, T) .
$$

Since, by construction all moments of $t_{i}^{*}(N, T)$ exist, conditioning on $\mathbf{W}_{f}$

$$
\operatorname{CIPS}^{*}(N, T)-\overline{C A D F}^{*}=o_{p}(1),
$$

where ${\overline{C A D F^{*}}}^{*}=N^{-1} \sum_{i=1}^{N} C A D F_{i}^{*}$ and

$$
C A D F_{i}^{*}= \begin{cases}C A D F_{i}, & \text { if }-K_{1}<C A D F_{i}<K_{2}, \\ -K_{1}, & \text { if } C A D F_{i} \leq-K_{1}, \\ K_{2}, & \text { if } C A D F_{i} \geq K_{2} .\end{cases}
$$

Under mild conditions stated in Pesaran (2007, Section 4), it can be shown that $\operatorname{CIPS}(N, T)$ and $\operatorname{CIPS}^{*}(N, T)$ converge almost surely to some limit distributions. These distributions are not analytically tractable, although they can be obtained easily by stochastic simulations, as will be described below, in which case the $\operatorname{CIPS}(N, T)$ and $\operatorname{CIP}^{*}(N, T)$ statistics are tabulated for different values of $k$. In what follows we only report the results for non-truncated version of the test statistics. The results for the truncated version are available upon request.

One could think of the $t_{i}(N, T)$ statistic to be analogous to the common Dickey-Fuller test statistic in the sense that the latter has a non-standard distribution, is free of nuisance parameters and the critical values depend on whether a trend term is included in the regression or not. The same applies to the $C I P S(N, T)$ and $\operatorname{CIPS}^{*}(N, T)$ statistics which are averages of the $t_{i}(N, T)$ statistic, and similarly their critical values depend on the nature of the deterministics and the number of $x$ 's in the ADF type regressions as a proxy for the unobserved components.

\subsection{The Case of Serially Correlated Errors}

As illustrated in Pesaran (2007) the residual serial correlation can be modeled in a number of different ways, directly via the idiosyncratic components, through the common effects or a mixture of the two. We focus on the first specification where cross section dependence is present under the multifactor error structure

$$
u_{i t}=\gamma_{i}^{\prime} \mathbf{f}_{t}+\zeta_{i t}
$$

and residual serial correlation is modeled as

$$
\zeta_{i t}=\theta_{i} \zeta_{i, t-1}+\eta_{i t}, \quad\left|\theta_{i}\right|<1, \text { for } i=1,2, \ldots, N
$$

where $\eta_{i t} \sim\left(0, \sigma_{i \eta}^{2}\right)$. 
In what follows we confine our attention to first order stationary processes for expositional convenience, though the analysis readily extends to higher order processes as well as to the alternative specifications of serial correlation mentioned above.

Under the above specification we have

$$
\Delta y_{i t}=\beta_{i}\left(y_{i, t-1}-\boldsymbol{\alpha}_{i}^{\prime} \mathbf{d}_{t-1}\right)+\boldsymbol{\alpha}_{i}^{\prime} \Delta \mathbf{d}_{t}+\boldsymbol{\gamma}_{i}^{\prime} \mathbf{f}_{t}+\zeta_{i t}\left(\theta_{i}\right)
$$

where $\zeta_{i t}\left(\theta_{i}\right)=\left(1-\theta_{i} L\right)^{-1} \eta_{i t}$. We also assume the coefficients of the autoregressive process to be homogeneous across $i$, although this could be relaxed at the cost of more complex mathematical details. Under the null that $\beta_{i}=0$, with $\theta_{i}=\theta$ and $\mathbf{d}_{t}=1$, (38) becomes

$$
\Delta y_{i t}=\gamma_{i}^{\prime} \mathbf{f}_{t}+\zeta_{i t}(\theta)
$$

or

$$
\Delta y_{i t}=\theta \Delta y_{i, t-1}+\gamma_{i}^{\prime}\left(\mathbf{f}_{t}-\theta \mathbf{f}_{t-1}\right)+\eta_{i t}
$$

Taking the first-difference of (7) and combining it with (39) we obtain in matrix notation

$$
\Delta \mathbf{Z}_{i}=\mathbf{F} \boldsymbol{\Gamma}_{i}^{\prime}+\Delta \mathbf{V}_{i}
$$

where $\Delta \mathbf{V}_{i}=\left(\boldsymbol{\zeta}_{i}^{\prime}(\theta), \Delta \mathbf{V}_{i x}^{\prime}\right)^{\prime}$ and $\boldsymbol{\zeta}_{i}(\theta)=\left(\zeta_{i 1}(\theta), \zeta_{i 2}(\theta), \ldots, \zeta_{i T}(\theta)\right)^{\prime}$, with the common factors $\mathbf{F}$, and factor loadings $\boldsymbol{\Gamma}_{i}$ defined as in the previous section. Taking cross section averages of (41) we have that

$$
\Delta \overline{\mathbf{Z}}=\mathbf{F} \overline{\boldsymbol{\Gamma}}^{\prime}+\Delta \overline{\mathbf{V}}
$$

where $\Delta \overline{\mathbf{V}}=N^{-1} \sum_{i=1}^{N} \Delta \mathbf{V}_{i}$, from which it follows under rank the condition (9) that

$$
\mathbf{F}=[\Delta \overline{\mathbf{Z}}-\Delta \overline{\mathbf{V}}] \overline{\mathbf{\Gamma}}\left(\overline{\boldsymbol{\Gamma}}^{\prime} \overline{\boldsymbol{\Gamma}}\right)^{-1}
$$

Thus in testing (4) we use the following cross sectionally augmented regression

$$
\Delta \mathbf{y}_{i}=b_{i} \mathbf{y}_{i,-1}+\overline{\mathbf{W}}_{i 1} \mathbf{h}_{i}+\epsilon_{i},
$$

where $\overline{\mathbf{W}}_{i 1}=\left(\Delta \mathbf{y}_{i,-1}, \Delta \overline{\mathbf{Z}}, \Delta \overline{\mathbf{Z}}_{-1}, \boldsymbol{\tau}_{T}, \overline{\mathbf{Z}}_{-1}\right)$, which is a $T \times(3 k+5)$ matrix.

The $t$-ratio of $\hat{b}_{i}$ in regression (44) is given by

$$
t_{i}(N, T)=\frac{\Delta \mathbf{y}_{i}^{\prime} \overline{\mathbf{M}}_{i 1} \mathbf{y}_{i,-1}}{\hat{\sigma}_{i}\left(\mathbf{y}_{i,-1}^{\prime} \overline{\mathbf{M}}_{i 1} \mathbf{y}_{i,-1}\right)^{1 / 2}}=\frac{\sqrt{T-(3 k+6)} \Delta \mathbf{y}_{i}^{\prime} \overline{\mathbf{M}}_{i 1} \mathbf{y}_{i,-1}}{\left(\Delta \mathbf{y}_{i}^{\prime} \overline{\mathbf{M}}_{i 1, p} \Delta \mathbf{y}_{i}\right)^{1 / 2}\left(\mathbf{y}_{i,-1}^{\prime} \overline{\mathbf{M}}_{i 1} \mathbf{y}_{i,-1}\right)^{1 / 2}}
$$

where $\overline{\mathbf{M}}_{i 1}=\mathbf{I}_{T}-\overline{\mathbf{W}}_{i 1}\left(\overline{\mathbf{W}}_{i 1}^{\prime} \overline{\mathbf{W}}_{i 1}\right)^{-1} \overline{\mathbf{W}}_{i 1}^{\prime}, \hat{\sigma}_{i}^{2}=[T-(3 k+6)]^{-1} \Delta \mathbf{y}_{i}^{\prime} \overline{\mathbf{M}}_{i 1, p} \Delta \mathbf{y}_{i}$ and $\overline{\mathbf{M}}_{i 1, p}=$ $\mathbf{I}_{T}-\mathbf{P}_{i 1}\left(\mathbf{P}_{i 1}^{\prime} \mathbf{P}_{i 1}\right)^{-1} \mathbf{P}_{i 1}^{\prime}, \mathbf{P}_{i 1}=\left(\overline{\mathbf{W}}_{i 1}, \mathbf{y}_{i,-1}\right)$.

Writing (40) in matrix notation and using (43) we have

$$
\Delta \mathbf{y}_{i}=\theta \Delta \mathbf{y}_{i,-1}+\left(\Delta \overline{\mathbf{Z}}-\theta \Delta \overline{\mathbf{Z}}_{-1}\right) \boldsymbol{\delta}_{i}+\sigma_{i \eta} \boldsymbol{v}_{i}
$$

with

$$
\boldsymbol{v}_{i}=\boldsymbol{\xi}_{i} / \sigma_{i \eta},
$$

where $\boldsymbol{\xi}_{i}=\left[\boldsymbol{\eta}_{i}-\left(\Delta \overline{\mathbf{V}}-\boldsymbol{\theta} \Delta \overline{\mathbf{V}}_{-1}\right) \boldsymbol{\delta}_{i}\right], \boldsymbol{\eta}_{i}=\left(\eta_{i 1}, \eta_{i 2}, \ldots, \eta_{i T}\right)^{\prime}$ and $E\left(\boldsymbol{\xi}_{i} \boldsymbol{\xi}_{i}^{\prime}\right)=\sigma_{i \eta}^{2} \mathbf{I}_{T}+O\left(N^{-1}\right)$. From (??) it follows that

$$
\mathbf{y}_{i,-1}=\stackrel{\circ}{y}_{i 0} \boldsymbol{\tau}_{T}+\overline{\mathbf{Z}}_{-1} \boldsymbol{\delta}_{i}+\sigma_{i \eta} \stackrel{\circ}{\mathfrak{s}}_{i \zeta,-1}
$$


where

$$
\stackrel{\mathfrak{s}}{i \zeta,-1}=\left[\mathbf{s}_{i \zeta,-1}-\overline{\mathbf{S}}_{\mathbf{v},-1} \boldsymbol{\delta}_{i}\right] / \sigma_{i \eta}
$$

$\mathbf{s}_{i \zeta,-1}=\left(0, s_{i \zeta 1}, \ldots, s_{i \zeta, T-1}\right)^{\prime}$ with $s_{i \zeta t}=\sum_{j=1}^{t} \zeta_{i j}(\theta), \overline{\mathbf{S}}_{\mathbf{v},-1}=\left(\overline{\mathbf{s}}_{\zeta,-1}, \overline{\mathbf{S}}_{\mathbf{v} x,-1}\right)$ with $\overline{\mathbf{s}}_{\zeta,-1}=$ $N^{-1} \sum_{i=1}^{N} \mathbf{s}_{i \zeta,-1}$ and $\stackrel{\circ}{y}_{i 0}=y_{i 0}-\boldsymbol{\delta}_{i}^{\prime}\left(\overline{\mathbf{z}}_{0}+\overline{\mathbf{v}}_{0}\right)$.

The test statistic (45) then becomes

$$
t_{i}(N, T)=\frac{\boldsymbol{v}_{i}^{\prime} \overline{\mathbf{M}}_{i 1} \stackrel{\circ}{\mathfrak{s}}_{i \zeta,-1}}{\left(\frac{\boldsymbol{v}_{i}^{\prime} \overline{\mathbf{M}}_{i 1, p} \boldsymbol{v}_{i}}{T-3 k-6}\right)^{1 / 2}\left(\stackrel{\mathfrak{s}}{\prime}_{i \zeta,-1}^{\prime} \overline{\mathbf{M}}_{i 1} \stackrel{\mathfrak{s}}{i \zeta,-1}^{1 / 2}\right.} .
$$

Theorem 2.3 Suppose the series $\mathbf{z}_{i t}$, for $i=1,2, \ldots, N, t=1,2, \ldots, T$, is generated under (4) according to (41) and $|\theta|<1$. Then under Assumptions $1-5$ and as $N$ and $T \rightarrow \infty, t_{i}(N, T)$ in (48) has the same sequential $(N \rightarrow \infty, T \rightarrow \infty)$ and joint $\left[(N, T)_{j} \rightarrow \infty\right]$ limit distribution given by (34) obtained for $\theta=0$.

Proof: See Appendix A.5.

For an $\operatorname{AR}(p)$ error specification in $(37)$, the relevant $t_{i}(N, T)$ statistic will be given by the OLS $t$-ratio of $b_{i}$ in the following $p^{t h}$ order augmented regression:

$$
\Delta \mathbf{y}_{i}=b_{i} \mathbf{y}_{i,-1}+\overline{\mathbf{W}}_{i p} \mathbf{h}_{i p}+\epsilon_{i}
$$

where $\overline{\mathbf{W}}_{i p}=\left(\Delta \mathbf{y}_{i,-1}, \Delta \mathbf{y}_{i,-2}, \ldots, \Delta \mathbf{y}_{i,-p} ; \Delta \overline{\mathbf{Z}}, \Delta \overline{\mathbf{Z}}_{-1}, \ldots, \Delta \overline{\mathbf{Z}}_{-p} ; \boldsymbol{\tau}_{T} ; \overline{\mathbf{Z}}_{-1}\right)$, which is a $T \times((k+$ 2) $(p+2)-1)$ matrix.

However, it is easily seen that the limit distribution of $t_{i}(N, T)$ with $N \rightarrow \infty$ for fixed $T$ depends on the augmentation order, $p$. Thus, we will obtain critical values of $t_{i}(N, T)$ for different choices of $p$.

\section{Critical Values}

The critical values of $C A D F_{i}$ and $\overline{C A D F}=N^{-1} \sum_{i=1}^{N} C A D F_{i}$ for different values of $k, N, T$ and lag-augmentation $p$, are obtained by stochastic simulation. Based on the results in Section 2 the limit distribution of $\overline{C A D F}$ does not depend on the factor loadings or $\sigma_{i}$. This implies that the distribution of the test statistic is invariant to the choice of $\boldsymbol{\Gamma}_{i}$ and $\sigma_{i}$ so long as $m \leq k+1$. Thus, without loss of generality we set $\boldsymbol{\Gamma}_{i}=\boldsymbol{\Gamma}=\mathbf{0}$, and $\sigma_{i}=\sigma=1$ in our stochastic simulations.

To be more precise, the $y_{i t}$ process is generated as

$$
y_{i t}=y_{i t-1}+u_{i t}, i=1,2, \ldots, N ; t=1,2, \ldots, T,
$$

where $u_{i t} \sim \operatorname{iidN}(0,1)$ with $y_{i 0}=0$. The $j^{\text {th }}$ element of the $k \times 1$ vector of the additional regressors $\mathbf{x}_{i t}$, is generated as

$$
x_{i j t}=x_{i j, t-1}+v_{i j t}, i=1,2, \ldots, N ; j=1,2, \ldots, k ; t=1,2, \ldots, T,
$$

with $v_{i j t} \sim i i d N(0,1)$ and $x_{i j 0}=0$. The $C A D F_{i}$ test statistic is calculated as the $t$-ratio of the coefficient on $y_{i t-1}$ of the regression of $\Delta y_{i t}$ on $y_{i t-1}, \overline{\mathbf{z}}_{t-1}^{\prime},\left(\Delta \mathbf{z}_{i . t-1}^{\prime}, \ldots, \Delta \mathbf{z}_{i . t-p}^{\prime}\right),\left(\Delta \overline{\mathbf{z}}_{t-1}^{\prime}, \ldots, \Delta \overline{\mathbf{z}}_{t-p}^{\prime}\right)$ where the following cases for the deterministics are entertained

Case I: no deterministics,

Case II: intercept only,

Case III: an intercept and a linear trend, 
and $E\left(C A D F_{i}\right)$ and $\operatorname{Var}\left(C A D F_{i}\right)$ are obtained as an average over all replications of $C A D F_{1}$ and the square of the standard deviation of $C A D F_{1}$ respectively, for $N, T=200$. The $\alpha \%$ critical values of the $C A D F_{1}$ and $\overline{C A D F}$ statistics are computed for $N, T=20,30,50,70,100,200$, $k+1=1,2,3,4$ and $p=0,1, \ldots, 4$, as the $1-\alpha$ quantiles of $C A D F_{1}$ and $\overline{C A D F}$ for $\alpha=$ $0.01,0.05,0.1$. Results for the critical values of the $\overline{C A D F}$ statistic are reported in Tables 1a-1c. The critical values for the individual statistics $C A D F_{i}$ are available upon request. All stochastic simulations are based on 10,000 replications.

\section{Small Sample Performance: Monte Carlo Evidence}

In what follows we investigate the small sample properties of the CIPS test defined by (35) by means of Monte Carlo experiments. Before doing so, we briefly present the panel unit root test statistics that we consider in our Monte Carlo alongside the CIPS test. These include the tests proposed by Im, Pesaran and Shin (IPS, 2003), Bai and Ng (2007), and Moon and Perron (2004). The IPS test is not valid under cross section dependence, but is inlcuded as a benchmark.

\subsection{Alternative Panel Unit Root Test Statistics}

The IPS test statistic is defined as

$$
I P S=\frac{\sqrt{N}\left\{t-b a r_{N T}-E\left(\tau_{T}\right)\right\}}{\sqrt{\operatorname{Var}\left(\tau_{T}\right)}}
$$

where $t$-bar ${ }_{N T}=N^{-1} \sum_{i=1}^{N} \tau_{i T}$, and $\tau_{i T}$ is the t-ratio of the $\operatorname{ADF}(p)$ regression of the $i^{\text {th }}$ cross section unit. $E\left(\tau_{T}\right)$ and $\operatorname{Var}\left(\tau_{T}\right)$ are the mean and variance of $\tau_{i T}$, which are listed in Table 3 of Im et al (2003).

Bai and $\mathrm{Ng}$ (2007) propose the $P_{b}$ and $P M S B$ tests, both of which are briefly described below. The former is the analog of the $t_{b}$ statistic of Moon and Perron (2004) except that it is based on a different set of residuals and the method of 'defactoring' of the data is different, while the latter is the panel version of the modified Sargan-Bhargava test. The $P_{b}$ and $P M S B$ tests are based on the so called PANIC residuals, which in the context of our notation as set out in Section 2, are obtained as follows. Firstly transform $y_{i t}: \underline{y}_{i t}=\Delta y_{i t}$ if $y_{i t}$ has individual effects, or $\underline{y}_{i t}=\Delta y_{i t}-\overline{\Delta y_{i}}$ with $\overline{\Delta y_{i}}=(T-1) \sum_{t=2}^{T} \Delta y_{i t}$, if $y_{i t}$ has a linear trend. Apply principal components to these transformed series to estimate $\mathbf{F}$, denoted as $\hat{\mathbf{F}}$, which is $\sqrt{T-1}$ times the $m$ eigenvectors corresponding to the first $m$ largest eigenvalues of the $(T-1) \times(T-1)$ matrix $\underline{\mathbf{Y}} \underline{\mathbf{Y}}^{\prime}$, where $\underline{\mathbf{Y}}=\left(\underline{\mathbf{y}}_{1}, \underline{\mathbf{y}}_{2}, \ldots, \underline{\mathbf{y}}_{N}\right)$ and $\underline{\mathbf{y}}_{i}=\left(\underline{y}_{i 2}, \underline{y}_{i 3}, \ldots, \underline{y}_{i T}\right)^{\prime}$. Under the normalisation $\hat{\mathbf{F}}^{\prime} \hat{\mathbf{F}} /(T-1)=\mathbf{I}_{m}$, the estimated factor loadings $\boldsymbol{\gamma}_{i}$ are $\hat{\boldsymbol{\gamma}}_{i}=\hat{\mathbf{F}}_{i}^{\prime} \underline{\mathbf{y}}_{i} /(T-1)$. Then obtain $\hat{u}_{i t}=\sum_{s=2}^{t} e_{i t}$, where $e_{i t}=\underline{y}_{i t}-\hat{\boldsymbol{\gamma}}_{i}^{\prime} \hat{\mathbf{f}}_{t}$.

Having obtained the PANIC residuals, the $P_{b}$ test is then based on a pooled estimate of the autoregressive coefficient $\rho$ in the following regression

$$
\hat{u}_{i t}=\rho \hat{u}_{i, t-1}+\epsilon_{i t} .
$$

The bias-corrected pooled PANIC autoregressive estimator for $\hat{u}_{i t}$ based on OLS estimation of (51) is given by 


$$
\hat{\rho}^{+}=\frac{\operatorname{tr}\left(\hat{\mathbf{U}}_{-1}^{\prime} \mathbf{M} \hat{\mathbf{U}}-N T \hat{\psi}\right)}{\operatorname{tr}\left(\hat{\mathbf{U}}_{-1}^{\prime} \mathbf{M} \hat{\mathbf{U}}_{-1}\right)}
$$

where $\hat{\mathbf{U}}$ are $(T-2) \times N$ matrices and $\hat{\psi}$ is the bias correction estimated from $\hat{\mathbf{E}}=\mathbf{M} \hat{\mathbf{U}}-\hat{\rho} \mathbf{M} \hat{\mathbf{U}}_{-1}$ with

$$
\hat{\rho}=\frac{\operatorname{tr}\left(\hat{\mathbf{U}}_{-1}^{\prime} \mathbf{M} \hat{\mathbf{U}}\right)}{\operatorname{tr}\left(\hat{\mathbf{U}}_{-1}^{\prime} \mathbf{M} \hat{\mathbf{U}}_{-1}\right)}
$$

and $\mathbf{M}=\mathbf{I}_{T-2}$ in the case of no deterministics or a constant only and $\mathbf{M}=\mathbf{I}_{T-1}-\mathbf{D}\left(\mathbf{D}^{\prime} \mathbf{D}\right)^{-1} \mathbf{D}^{\prime}$ in the case of an intercept and a linear trend, where $\mathbf{D}=\left(\mathbf{d}_{1}, \ldots, \mathbf{d}_{T-1}\right)^{\prime}$ and $\mathbf{d}_{t}=(1 t)^{\prime}$.

The $P_{b}$ statistic is defined as

$$
P_{b}=\sqrt{N} T\left(\hat{\rho}^{+}-1\right) \sqrt{\frac{1}{N T^{2}} \operatorname{tr}\left(\hat{\mathbf{U}}_{-1} \mathbf{M} \hat{\mathbf{U}}_{-1}^{\prime}\right) K_{b} \frac{\hat{\omega}^{2}}{\hat{\phi}_{\epsilon}^{4}}} .
$$

The bias adjustment depends on the treatment of the deterministic terms such that in the case of no deterministics or a constant only $\left(\hat{\psi}, K_{b}\right)=\left(\hat{\lambda}_{\epsilon}, 1\right)$, and $\left(\hat{\psi}, K_{b}\right)=\left(-\hat{\sigma}_{\epsilon}^{2} / 2,4\right)$ in the case of an intercept and a linear trend, where $\mathbf{M}$ is defined as above. The parameters $\hat{\sigma}_{\epsilon}^{2}, \hat{\omega}^{2}, \hat{\lambda}_{\epsilon}$ and $\hat{\phi}_{\epsilon}^{4}$, are the limits of the cross section averages of the short and long run variance, the one-sided long run variance and the limit of the cross section averages of the square of the long run variance, respectively.

The $P M S B$ statistic is defined as

$$
P M S B=\frac{\sqrt{N}\left(\operatorname{tr}\left(\frac{1}{N T^{2}} \hat{\mathbf{U}}^{\prime} \hat{\mathbf{U}}\right)-\hat{\psi}\right.}{\sqrt{\hat{\phi}_{\epsilon}^{4} / K_{m s b}}}
$$

where as above the bias correction depends on the deterministic trends. In the case of no deterministics or a constant only $\left(\hat{\psi}, K_{m s b}\right)=\left(\hat{\omega}_{\epsilon} / 2,3\right)$, and $\left(\hat{\psi}, K_{m s b}\right)=\left(\hat{\omega}_{\epsilon} / 6,45\right)$ in the case of an intercept and a linear trend.

To compute the $t_{b}^{*}$ test statistic proposed by Moon and Perron (2004), initially the pooled OLS estimator is obtained from a first order autoregressive model of the observed data. A factor model is then estimated using the residuals computed based on the pooled OLS estimator and the factor loadings are obtained. The bias-corrected defactored pooled OLS estimator is then defined similar to (52) above where $\hat{\mathbf{U}}$ is replaced by the de-factored panel data obtained by projecting the panel data onto the space orthogonal to the (estimated) factor loadings. The nuisance parameters are defined on the residuals of the defactored data where the long-run variance is estimated by employing Andrews and Monahan's (1992) estimator based on the quadratic spectral kernel and prewhitening.

All the above test statistics are asymptotically distributed as standard normal so that they all reject the null hypothesis if they are less than -1.645 , at the $5 \%$ significance level. For further details see Bai and Ng (2007) and Moon and Perron (2004).

For the test procedures proposed by Bai and Ng (2007) and Moon and Perron (2004) that require the estimation of the number of factors, information criteria proposed by Bai and $\mathrm{Ng}$ (2002) are typically used, initialised by specification of the maximum number of factors. In what follows we consider experiments where the number of factors is treated as known, as well as experiments where the number of factors are estimated. In the latter case we use the BIC3 
criterion of Bai and $\mathrm{Ng}$ (2002) which is favoured by these authors as more robust in the presence of cross section correlation in the idiosyncratic errors.

Finally, it is worth noting that we do not consider the $P_{a}$ and $t_{a}^{*}$ tests of Bai and $\mathrm{Ng}$ (2007) and Moon and Perron (2004), respectively, as these tests do not perform as well as the alternative $P_{b}$ and $t_{b}^{*}$ tests.

\subsection{Monte Carlo Design}

Initially we shall consider dynamic panel models with fixed effects and a two-factor error structure. The data generating process (DGP) is given by

$$
y_{i t}=\left(1-\rho_{i}\right) \alpha_{i}+\rho_{i} y_{i, t-1}+\gamma_{i 1} f_{1 t}+\gamma_{i 2} f_{2 t}+\varepsilon_{i t}, i=1,2, \ldots, N ; t=-49, \ldots, T
$$

with $y_{i,-50}=0$, where $\alpha_{i} \sim \operatorname{iidN}(1,1)$,

$$
f_{j t}=\rho_{f j} f_{f j, t-1}+\varpi_{j t}, \varpi_{j t} \sim i i d N(0,1),
$$

with $f_{j,-50}=0$ and $\rho_{f j}=0$ for $j=1,2$, and

$$
\varepsilon_{i t}=\rho_{i \varepsilon} \varepsilon_{i t-1}+\zeta_{i t}, \zeta_{i t} \sim i i d N\left(0, \sigma_{i}^{2}\right)
$$

with $\varepsilon_{i,-50}=0$ and $\rho_{i \varepsilon}=\rho_{\varepsilon}=0, \sigma_{i}^{2} \sim i i d U[0.5,1.5]$. The $x_{i t}$ process is generated as

$$
x_{i t}=\mu_{i}+\gamma_{i x 1} f_{1 t}+\gamma_{i x 2} f_{2 t}+v_{i x t}, i=1,2, \ldots, N ; t=-49, \ldots, T
$$

with $x_{i,-50}=0$, where $\mu_{i} \sim \operatorname{iidN}(1,1)$, and

$$
v_{i x t}=v_{i x t-1}+e_{i v x t}, \quad e_{i v x t}=\rho_{i v x} e_{i v x, t-1}+\varrho_{i t}, \varrho_{i t} \sim i i d N(0,1)
$$

with $v_{i x,-50}=0, e_{i v x,-50}=0$, and $\rho_{i v x} \sim i i d U[0.2,0.4]$. The factor loadings are generated as $\gamma_{i 1} \sim i i d U[1,3], \gamma_{i 2} \sim i i d U[0,2], \gamma_{i x 1} \sim i i d U[0,2], \gamma_{i x 2} \sim i i d U[1,3]$, so that

$$
E\left(\boldsymbol{\Gamma}_{i}\right)=\left(\begin{array}{ll}
2 & 1 \\
1 & 2
\end{array}\right)
$$

and the rank condition (9) is satisfied.

We consider three combinations of serially correlated errors: (A) serially uncorrelated $\varepsilon_{i t}$ and $f_{j t}\left(\rho_{i \varepsilon}=\rho_{\varepsilon}=0\right.$ and $\left.\rho_{\varphi 1}=\rho_{\varphi 2}=0\right)$; (B) serially correlated $\varepsilon_{i t}\left(\rho_{i \varepsilon} \sim i i d U[0.2,0.4]\right.$ whilst $\left.\rho_{\varphi 1}=\rho_{\varphi 2}=0\right) ;(\mathrm{C})$ serially correlated $f_{j t}\left(\rho_{i \varepsilon}=\rho_{\varepsilon}=0\right.$ whilst $\left.\rho_{f 1}=\rho_{f 2}=0.3\right) . \Delta v_{i x t}$ are serially correlated in all experiments $\left(\rho_{i v x} \sim i i d U[0.2,0.4]\right)$.

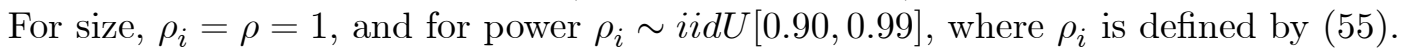

In order to examine the effect of misspecification of the number of factors, in one set of experiments, the DGP contains two factors but tests are carried out assuming there are three factors. Specifically, the DGP is the same as in experiment (A), $\varepsilon_{i t}$ and $f_{j t}$ are not serially correlated, except $\gamma_{i 2}=\gamma_{2}=0$ and $\gamma_{i x 2}=\gamma_{x 2}=0$, but $\gamma_{i x 1} \sim i i d U[1,3]$.

Similar sets of experiments are carried out for the model with a linear time trend. The DGP corresponding to (53) and (56) are

$$
y_{i t}=\alpha_{i}+\left(1-\rho_{i}\right) \delta_{i} t+\rho_{i} y_{i, t-1}+\gamma_{i 1} f_{1 t}+\gamma_{i 2} f_{2 t}+\varepsilon_{i t}, i=1,2, \ldots, N ; t=-49, \ldots, T
$$




$$
x_{i t}=\mu_{i}+\lambda_{i} t+\gamma_{i x 1} f_{1 t}+\gamma_{i x 2} f_{2 t}+v_{i x t}, i=1,2, \ldots, N ; t=-49, \ldots, T
$$

respectively, where $\delta_{i} \sim i i d[0.0,0.02]$ and $\lambda_{i} \sim i i d[0.0,0.02]$.

Other variables are defined as above. $\alpha_{i}, \gamma_{i 1}, \gamma_{i 2}, \rho_{f j}, \rho_{i}, \mu_{i}, \gamma_{i x 1}, \gamma_{i x 2}, \rho_{i v x}, \delta_{i}$ and $\lambda_{i}$ are drawn once and fixed over the replications. All tests are conducted at the $5 \%$ significance level. All combinations of $N, T=20,30,50,70,100,200$ are considered, and all experiments are based on 2,000 replications.

\subsection{Results}

Size and power of the tests in the case of the experiments with an intercept only are summarised in Tables 2-10. Recall that for all experiments the first difference of the augmeted variables, $\Delta \mathbf{x}_{i t}$, are serially correlated and the models contain two factors, except the design for Tables 7-10. Also note that in the case of serially correlated idiosyncratic errors $\varepsilon_{i t}$, or factors, lag augmentation is required only for the CIPS test as the $P_{b}$ and $P M S B$ of Bai and $\mathrm{Ng}$ (2007) and the $t_{b}^{*}$ test of Moon and Perron (2004) are based on an estimate of the long-run variance.

Table 2 provides the results for the model where both factors, $\left(f_{1 t}, f_{2 t}\right)$, and the idiosyncratic components, $\varepsilon_{i t}$, are not serially correlated. It is clear that in all the experiments the IPS test over-rejects the null for all combinations of $N$ and $T$. The $P_{b}$ test of Bai and $\mathrm{Ng}$ (2007) tends to over-reject slightly, with the extent of over-rejection rising as $N$ increases for fixed $T$. Conversely, the $P M S B$ test tends to under-reject particularly for small $N$ and $T$. The $t_{b}^{*}$ test greatly overrejects unless $T$ is much larger than $N$. On the contrary, the CIPS test has the correct size for all combinations of sample sizes, even when $T$ is very small relative to $N$. In terms of power, the CIPS test is less powerful than the pooled $P_{b}$ and $P M S B$ tests for smaller values of $T$ as one would probably expect, while it tends to be more powerful for large $N$ and $T$.

Tables 3 and 4 present the results for the case where $\varepsilon_{i t}$ are serially correlated but $f_{1 t}$ and $f_{2 t}$ are not. Without time series augmentation, the CIPS test shows significant size distortions. With augmentation the size and the power properties of the CIPS test are similar to those reported in Table 2. The $P_{b}$ statistic continues to display the tendency to over-reject, more so in the case of negative serial correlation in the error terms, while the PMSB test is severly undersized in the case of positive serial correlation and severly oversized in the case of negative serial correlation for all combinations of $N$ and $T$. The $t_{b}^{*}$ test shows similar size performance to that in Table 2 for positive serial correlation, though somewhat more exaggerated in the negative serial correlated case.

Tables 5 and 6 provide the results for the model where $f_{1 t}$ and $f_{2 t}$ are serially correlated but $\varepsilon_{i t}$ is not. The $P_{b}$ and CIPS tests, and to a lesser extent the PMSB test, exhibit size distortions unless $T$ is sufficiently large relative to $N$, as predicted by theory for the CIPS test. The extent of over-rejection of the CIPS test is less than that of the $P_{b}$ test, and is reduced with time series augmentation. The performance of the $t_{b}^{*}$ test is similar to what was reported for this test in the previous experiments.

Tables 7-10 report the results for the model where there are two factors in the DGP, but tests are incorrectly conducted assuming there are three factors. In particular, Tables 7 and 8 report results for the case where the number of factors is treated as known for the $P_{b}, P M S B$ and $t_{b}^{*}$ tests, while Tables 9 and 10 consider the case where the number of factors for these statistics is estimated using the BIC3 criterion of Bai and $\mathrm{Ng}$ (2002) and setting the maximum number of factors to be three. The results suggest that this type of misspecification does not affect the CIPS test very much, as predicted by our asymptotic theory. The slight tedency of 
the CIPS test to over-reject for small $T$ seems to be due to the additional lag terms included to deal with the assumed additional factor. The same applies to the $P_{b}$ test which displays similar performance to that in Table 2, whether the number of factors is treated as known and equal to three or is estimated. For the $P M S B$ and $t_{b}^{*}$ tests, their performance is closer to that in Table 2 when the number of factors are estimated else their tendency to over-reject is larger. For the latter three statistics, in most cases power is higher when the number of factors is treated as known compared to when estimated, and is generally greater than that of the CIPS test.

Monte Carlo results for the case of an intercept and a linear trend are summarised in Tables 11-19, the experimental designs of which correspond to the ones that underlie the results in Tables 2-10. The $P_{b}$ test severely over-rejects in all experiments, apart from the case of positive serial correlation with a large $T$. On the whole, the $P M S B$ statistic under-rejects except for the case of negative serial correlation when $T$ is not too large, while in this case there is also the tendency to over-reject for large $N$. The size distortion of the $t_{b}^{*}$ test is worse in the case of a linear trend in almost all experiments, with the power declining substantially as $T$ increases. This is consistent with the analysis of Moon and Perron (2004), who show that their test has no power in the case of a linear trend. For the case where there are two factors, while tests are conducted under the belief of the existence of three factors, or estimated setting the maximum number of factors to three for the $P_{b}, P M S B$ and $t_{b}^{*}$ statistics, the behaviour of the $P_{b}$ and $P M S B$ tests in terms of size is similar to that reported in Tables 7 and 9 . It is only for the $t_{b}^{*}$ test that the size distortion decreases when the number of factors is estimated. The performance of the CIPS test for the experiments with an intercept and a linear trend is very similar to the intercept only case, which has the CIPS test controlling well for size in almost all cases and power reasonably high particulalrly for larger $T$ and $N$.

For better small sample power properties of the CIPS test one could also consider cross section augmented versions of unit root tests due to Elliott et al. (1996), Fuller and Park (1995), and Leybourne (1995) as in Smith et al. (2004).

\section{Empirical Application}

As an illustration of the proposed test we consider the application of panel unit root tests to the Fisher parity, namely the difference between the nominal short term interest rate and inflation, and to the real equity price across $N=32$ and $N=26$ countries, respectively, using quarterly observations over the period 1979Q2-2003Q4 (i.e. $T=99$ ). Existing evidence on the validity of the Fisher parity is rather mixed. The second application is chosen since it is generally believed that real equity prices are non-stationary and it would be interesting to see if the outcomes of the tests considered in this paper are in line with this belief.

The table below shows the dependent variable, $y_{i t}$, and the additional regressors, $\mathbf{x}_{i t}$, considered for both applications:

\begin{tabular}{|c|c|c|}
\hline & $y_{i t}$ & $\mathbf{x}_{i t}$ \\
\hline Fisher Parity $(N=32)$ & $r_{i t}^{S}-\pi_{i t}$ & $\left(g d p_{i t}, e q_{i t}, e p_{i t}, r_{i t}^{L}, p_{o i l}\right)^{\prime}$ \\
Real Equity Prices $(N=26)$ & $e q_{i t}$ & $\left(g d p_{i t}, \pi_{i t}, e p_{i t}, r_{i t}^{S}, r_{i t}^{L}, p o i l_{t}\right)^{\prime}$ \\
\hline
\end{tabular}

where

$$
\begin{gathered}
g d p_{i t}=\ln \left(G D P_{i t} / C P I_{i t}\right), \quad p_{i t}=\ln \left(C P I_{i t}\right), e q_{i t}=\ln \left(E Q_{i t} / C P I_{i t}\right), e_{i t}=\ln \left(E_{i t}\right), \\
r_{i t}^{S}=0.25 * \ln \left(1+R_{i t}^{S} / 100\right), \quad r_{i t}^{L}=0.25 * \ln \left(1+R_{i t}^{L} / 100\right), \quad \text { poil } \\
t
\end{gathered}
$$


with $G D P_{i t}$ the nominal Gross Domestic Product of country $i$ during period $t$ in domestic currency, $C P I_{i t}$ the consumer price index in country $i$ at time $t, E Q_{i t}$ the nominal equity price index, $E_{i t}$ the nominal exchange rate of country $i$ in terms of $U . S$. dollars, $R_{i t}^{S}$ is the short rate of interest per annum in per cent (typically a three month rate), $R_{i t}^{L}$ the long rate of interest per annum in per cent (typically the yields on ten year government bonds), and $P O I L_{t}$ is the price of Brendt Crude oil, so that $r_{i t}^{S}$ is the quarterly short-term interest rate, $\pi_{i t}=\left(p_{i t}-p_{i, t-1}\right)$ the quarterly inflation rate, $e p_{i t}=e_{i t}-p_{i t}$ is the real exchange rate, $e q_{i t}$ is the real equity price index, $g d p_{i t}$ is real output, $r_{i t}^{L}$ is the quarterly long-term interest rate and poil $_{t}$ is the nominal oil price. ${ }^{5}$

The 32 countries considered are: Argentina, Australia, Austria, Belgium, Brazil, Canada, Chile, China, France, Finland, Germany, Indonesia, India, Italy, Japan, Korea, Malaysia, Mexico, Netherlands, New Zealand, Norway, Peru, Philippines,Spain, Sweden, Switzerland, Singapore, South Africa, Thailand, Turkey, UK. Note that not all $\mathbf{x}_{i t}$ variables are available for all countries due to data constraints. In particular there are 32 series for $g d p_{i t}, 26$ series for $e q_{i t}$, 31 series for $e p_{i t}$ (excluding the US), and 18 series for $r_{i t}^{L}$.

To begin with we apply the information criteria of Bai and $\mathrm{Ng}(2002)$ to the $y_{i t}$ variables of interest. For the Fisher parity, the use of Bai-Ng information criteria led to mixed outcomes, and we thought it reasonable to infer from the results that the maximum number of factors is $m_{\max }=4$. Not knowing the true number of factors, we present results for $m=1,2,3$ and 4 in the case of the $P_{b}, P M S B$ and $t_{b}^{*}$ tests. For the real equity series applying the information criteria of Bai and $\mathrm{Ng}$ (2002) was not very helpful as the number of factors always turned out to be the maximum set. We therefore also set $m_{\max }=4$ for this application.

The additional series, $\mathbf{x}_{i t}$, can be either used directly or, as mentioned earlier, one could use the cumulated principle components of their first differences. Given the number of $\mathbf{x}^{\prime} s$ considered as defined in (60) above, we follow the latter approach. In particular, we pool the $\mathbf{x}_{i t}$ data across all countries $(N=32$ for Fisher equation and $N=26$ for the real equity prices) and consider the first differences, $\Delta \mathbf{x}_{i t}$. Principle component approach is then applied to the demeaned and standardised values of $\Delta \mathbf{x}_{i t}$ 's, and the resulting eigenvectors are cumulated and used as the common factors, $\overline{\mathbf{x}}_{t}{ }^{6}$ These are appended to the cross section average of the original series of interest, $\bar{y}_{t}$, to form $\overline{\mathbf{z}}_{t}=\left(\bar{y}_{t}, \overline{\mathbf{x}}_{t}^{\prime}\right)^{\prime}$ which are used to augment the panel unit root regression associated with the CIPS test as described in Section $2 .^{7}$

Tables 20 and 21 present the results for the proposed panel unit root test including the $P_{b}$, $P M S B$ and $t_{b}^{*}$ statistics, at the $5 \%$ significance level, considering a maximum lag order, $p_{\max }$, of four for the unit root regressions, where applicable. Note that for the Fisher equation a constant only is considered in the regressions, while for real equity prices both a constant and a linear trend are included.

Results of the panel unit root statistics for testing the null hypothesis of a unit root in the Fisher parity across 32 countries are given in Table 20. It appears that for all values of the autoregressive lag order $p$, the CIPS test rejects the joint null hypothesis of a unit root and no cross section cointegration at the $5 \%$ level. The $P_{b}, P M S B$ and $t_{b}^{*}$ statistics also reject the

\footnotetext{
${ }^{5}$ For a detailed description of the data and sources see Supplement A of Dees, di Mauro, Pesaran and Smith (2007).

${ }^{6}$ Applying the information criteria of Bai and $\mathrm{Ng}$ (2002) to determine the number of factors for the pooled $\mathbf{x}^{\prime} s$ did not prove very helpful, since as in the case of the equity price series, $y_{i t}$, the number of factors always turned out to be the maximum set.

${ }^{7}$ We also applied cointegration tests to the principal components of the $\mathbf{x}^{\prime} s$ and found that we could not reject the null of zero rank based on a $\operatorname{VAR}(4)$.
} 
null hypothesis for all values of $m$, which suggests that at least for some countries the Fisher parity holds. These results are in line with the panel cointegration tests applied to the Fisher equation. See, for example, Westerlund (2007).

Turning to the results for the case of the real equity prices, the CIPS test does not reject the joint null hypothesis at the $5 \%$ level for all $k$ and $p$. Similarly, the $P_{b}$ and $t_{b}^{*}$ statistics also do not reject the null hypothesis for all values of $m$. Contrary to these results, the PMSB test strongly rejects the unit root hypothesis in real equity prices if two or more unobserved common factors are assumed. This result does not accord with the generally accepted view that real equity prices approximately follow random walks with a drift.

Table 20. Panel Unit Root Test Statistics for Testing the Null Hypothesis of a Unit Root in the Fisher Parity Across 32 Countries Over the Period 1979Q2 - 2003Q4

\begin{tabular}{|c|c|c|c|c|c|c|c|c|}
\hline \multirow[b]{2}{*}{$p$} & \multicolumn{4}{|c|}{$m=1$} & \multicolumn{4}{|c|}{$m=2$} \\
\hline & 1 & 2 & 3 & 4 & 1 & 2 & 3 & 4 \\
\hline CIPS & $-4.80^{\dagger}$ & $-3.95^{\dagger}$ & $-3.00^{\dagger}$ & $-2.90^{\dagger}$ & $-4.90^{\dagger}$ & $-4.05^{\dagger}$ & $-3.01^{\dagger}$ & $-2.91^{1}$ \\
\hline$P_{b}$ & \multicolumn{4}{|c|}{$-10.58^{\dagger}$} & \multicolumn{4}{|c|}{$-3.91^{\dagger}$} \\
\hline$P M S B$ & \multicolumn{4}{|c|}{$59.02^{\dagger}$} & \multicolumn{4}{|c|}{$-77.90^{\dagger}$} \\
\hline$t_{b}^{*}$ & \multicolumn{4}{|c|}{$-17.66^{\dagger}$} & \multicolumn{4}{|c|}{$-17.49^{\dagger}$} \\
\hline & \multicolumn{4}{|c|}{$m=3$} & \multicolumn{4}{|c|}{$m=4$} \\
\hline$p$ & 1 & 2 & 3 & 4 & 1 & 2 & 3 & 4 \\
\hline CIPS & $-4.84^{\dagger}$ & $-3.90^{\dagger}$ & $-2.91^{\dagger}$ & $-2.70^{\dagger}$ & $-5.12^{\dagger}$ & $-4.20^{\dagger}$ & $-3.18^{\dagger}$ & -2.89 \\
\hline$P_{b}$ & \multirow{2}{*}{\multicolumn{4}{|c|}{$\begin{array}{l}-3.26^{\dagger} \\
-17523^{\dagger}\end{array}$}} & \multicolumn{4}{|c|}{$-5.17^{\dagger}$} \\
\hline$P M S B$ & & & & & & -31 & & \\
\hline$t_{b}^{*}$ & \multicolumn{4}{|c|}{$-21.27^{\dagger}$} & \multicolumn{4}{|c|}{$-25.11^{\dagger}$} \\
\hline
\end{tabular}

Note: ${ }^{\dagger}$ denotes rejection at the $5 \%$ significance level. $P_{b}$ and $P M S B$ are the Bai and $\mathrm{Ng}$ (2007) statistics and $t_{b}^{*}$ is that of Moon and Perron (2004). $m$ denotes the number of factors used. All three tests reject the null hypothesis of a unit root if they are less than -1.645. They adopt automatic lag-order selection for the estimation of long-run variances, which explains why only one value is reported for these tests. The critical values of the CIPS test for values of $p=1$ to 4 lie in the range $[-2.15,-2.09]$ for $k=0,[-2.40,-2.31]$ for $k=1,[-2.61,-2.47]$ for $k=2$, $[-2.78,-2.60]$ for $k=3$. 
Table 21. Panel Unit Root Test Statistics for Testing Null Hypothesis of a Unit Root in Real Equity Prices Across 26 Countries Over the Period 1979Q2 - 2003Q4

\begin{tabular}{|c|c|c|c|c|c|c|c|c|}
\hline \multirow[b]{2}{*}{$p$} & \multicolumn{4}{|c|}{$m=1$} & \multicolumn{4}{|c|}{$m=2$} \\
\hline & 1 & 2 & 3 & 4 & 1 & 2 & 3 & 4 \\
\hline CIPS & -2.22 & -2.40 & -2.59 & -2.58 & -2.50 & -2.63 & -2.75 & -2.72 \\
\hline$P_{b}$ & \multicolumn{4}{|c|}{0.08} & \multicolumn{4}{|c|}{-0.86} \\
\hline$P M S B$ & \multicolumn{4}{|c|}{-1.24} & \multicolumn{4}{|c|}{$-1.74^{\dagger}$} \\
\hline$t_{b}^{*}$ & \multicolumn{4}{|c|}{-0.17} & \multicolumn{4}{|c|}{-0.89} \\
\hline & \multicolumn{4}{|c|}{$m=3$} & \multicolumn{4}{|c|}{$m=4$} \\
\hline$p$ & 1 & 2 & 3 & 4 & 1 & 2 & 3 & 4 \\
\hline CIPS & -2.58 & -2.59 & -2.54 & -2.39 & -2.60 & -2.58 & -2.50 & -2.24 \\
\hline$P_{b}$ & \multicolumn{4}{|c|}{-1.13} & \multicolumn{4}{|c|}{-1.34} \\
\hline$P M S B$ & \multicolumn{4}{|c|}{$-1.69^{\dagger}$} & \multicolumn{4}{|c|}{$-1.82^{\dagger}$} \\
\hline$t_{b}^{*}$ & \multicolumn{4}{|c|}{-1.36} & \multicolumn{4}{|c|}{-1.49} \\
\hline
\end{tabular}

Note: ${ }^{\dagger}$ denotes rejection at the 5\% significance level. $P_{b}$ and $P M S B$ are the Bai and $\mathrm{Ng}$ (2007) statistics and $t_{b}^{*}$ is that of Moon and Perron (2004). All three tests reject the null hypothesis of a unit root if they are less than -1.645. They adopt automatic lag-order selection for the estimation of long-run variances, which explains why only one value is reported for these tests. The critical values of the CIPS test for values of $p=1$ to 4 lie in the range $[-2.65,-2.60]$ for $k=0,[-2.85,-2.75]$ for $k=1,[-3.01,-2.85]$ for $k=2,[-3.15,-2.94]$ for $k=3$.

\section{Concluding Remarks}

This paper considers a simple panel unit root test that is valid in the presence of cross section dependence induced by multiple common factors. This is achieved by making use of additional observed variables that share the same common factors. The tests are based on the average of t-ratios from an ADF regression of the series of interest augmented by the cross section averages of the dependent variable as well as the additional $k$ regressors.

Most importantly, our test procedure only requires specification of the maximum number of factors, in contrast to other panel unit root tests based on principal components that require in addition the estimation of the number of factors as well as the factors themselves. So long as $k+1 \geq m$, asymptotically our proposed unit root test, does not depend on any nuisance parameters and though non-standard, critical values can be calculated easily by simulation. Small sample properties of the proposed test are investigated by Monte Carlo experiments, which suggest that for both an intercept only and an intercept and linear trend included in the regression, it controls well for size in almost all cases, especially in the presence of serial correlation in the error terms, contrary to the tests of Bai and $\mathrm{Ng}$ (2007) and Moon and Perron (2004). Furthermore, it is fairly robust to misspecification of the true number of factors. In terms of power, out test appears to exhibit higher power compared to alternative tests for large $T$ and $N$, both in the case of an intercept only and in the case of an intercept and a linear trend.

The panel unit root tests are applied to real interest rates (Fisher's inflation parity) and real equity prices across countries. All tests reject a unit root in real interest rates which is line with panel cointegration tests of the Fisher equation. However, for real equity prices, the most reliable test of Bai and $\mathrm{Ng}$ (2007) in the case of a linear trend, rejects the unit root hypothesis in real equity prices if two or more unobserved common factors are assumed. This is in contrast 
to the results of the other panel unit root tests considered in this paper, and does not accord with the generally accepted view that real equity prices approximately follow random walks with a drift.

\section{Appendix A: Mathematical Proofs}

\section{A.2 Preliminary Order Results}

The results shown below are for the serially uncorrelated case. For the serially correlated case, analogous order results are obtained.

\section{A.2.1 Order Results A}

Under Assumptions 1-5,

$$
\begin{gathered}
\frac{\boldsymbol{\varepsilon}_{i}^{\prime} \bar{\varepsilon}}{T}=O_{p}\left(\frac{1}{\sqrt{N T}}\right), \frac{\boldsymbol{\varepsilon}_{i}^{\prime} \Delta \overline{\mathbf{V}}}{T}=O_{p}\left(\frac{1}{\sqrt{T N}}\right), \\
\frac{\boldsymbol{\tau}_{T}^{\prime} \overline{\boldsymbol{\varepsilon}}}{T}=O_{p}\left(\frac{1}{\sqrt{N T}}\right), \frac{\boldsymbol{\tau}_{T}^{\prime} \Delta \overline{\mathbf{V}}}{T}=O_{p}\left(\frac{1}{\sqrt{N T}}\right), \\
\frac{\overline{\boldsymbol{\varepsilon}}^{\prime} \Delta \overline{\mathbf{V}}}{T}=O_{p}\left(\frac{1}{\sqrt{T} N}\right), \\
\frac{\overline{\boldsymbol{\varepsilon}}^{\prime} \overline{\boldsymbol{\varepsilon}}}{T}=O_{p}\left(\frac{1}{N}\right), \frac{\Delta \overline{\mathbf{V}}^{\prime} \Delta \overline{\mathbf{V}}}{T}=O_{p}\left(\frac{1}{N}\right), \\
\frac{\mathbf{F}^{\prime} \bar{\varepsilon}}{T}=O_{p}\left(\frac{1}{\sqrt{T N}}\right), \frac{\mathbf{F}^{\prime} \Delta \overline{\mathbf{V}}}{T}=O_{p}\left(\frac{1}{\sqrt{T N}}\right) .
\end{gathered}
$$

\section{A.2.2 Order Results B}

Under Assumptions 1-5,

$$
\begin{gathered}
\frac{\overline{\mathbf{S}}_{\mathbf{v},-1}^{\prime} \Delta \overline{\mathbf{V}}}{T}=O_{p}\left(\frac{1}{N}\right), \\
\frac{\overline{\mathbf{S}}_{\mathbf{v},-1}^{\prime} \boldsymbol{\varepsilon}_{i}}{T}=O_{p}\left(\frac{1}{\sqrt{N}}\right), \frac{\overline{\mathbf{S}}_{\mathbf{v},-1}^{\prime} \mathbf{F}}{T}=O_{p}\left(\frac{1}{\sqrt{N}}\right), \\
\frac{\overline{\mathbf{s}}_{-1}^{\prime} \boldsymbol{\tau}_{T}}{T}=O_{p}\left(\sqrt{\frac{T}{N}}\right), \frac{\overline{\mathbf{S}}_{\mathbf{v},-1}^{\prime} \boldsymbol{\tau}_{T}}{T}=O_{p}\left(\sqrt{\frac{T}{N}}\right), \\
\frac{\overline{\mathbf{S}}_{\mathbf{v},-1}^{\prime} \overline{\mathbf{S}}_{\mathbf{v},-1}}{T^{2}}=O_{p}\left(\frac{1}{N}\right), \frac{\overline{\mathbf{S}}_{\mathbf{v},-1}^{\prime} \overline{\mathbf{s}}_{-1}}{T^{2}}=O_{p}\left(\frac{1}{N}\right), \\
\frac{\mathbf{s}_{i,-1}^{\prime} \overline{\mathbf{S}}_{\mathbf{v},-1}}{T^{2}}=O_{p}\left(\frac{1}{\sqrt{N}}\right), \frac{\mathbf{S}_{f,-1}^{\prime} \overline{\mathbf{S}}_{\mathbf{v},-1}}{T^{2}}=O_{p}\left(\frac{1}{\sqrt{N}}\right), \\
\frac{\mathbf{s}_{i,-1}^{\prime} \Delta \overline{\mathbf{V}}}{T}=O_{p}\left(\frac{1}{\sqrt{N}}\right), \frac{\mathbf{S}_{f,-1}^{\prime} \Delta \overline{\mathbf{V}}}{T}=O_{p}\left(\frac{1}{\sqrt{N}}\right) .
\end{gathered}
$$

\section{A.2.3 Order Results C}

Recall that $\boldsymbol{v}_{i}=\left(\boldsymbol{\varepsilon}_{i}-\Delta \overline{\mathbf{V}} \boldsymbol{\delta}_{i}\right) / \sigma_{i}$ and ${ }^{\circ}{ }_{i,-1}=\left(\mathbf{s}_{i,-1}-\overline{\mathbf{S}}_{\mathbf{v},-1} \boldsymbol{\delta}_{i}\right) / \sigma_{i}$. Thus, from (13) and (23) we have

$$
\Delta \overline{\mathbf{Z}}=\mathbf{F} \overline{\boldsymbol{\Gamma}}^{\prime}+\Delta \overline{\mathbf{V}}
$$

and

$$
\overline{\mathbf{Z}}_{-1}=\boldsymbol{\tau}_{T} \otimes \overline{\mathbf{z}}_{0}^{\prime}+\mathbf{S}_{f,-1} \overline{\boldsymbol{\Gamma}}^{\prime}+\overline{\mathbf{S}}_{\mathbf{v},-1}
$$


Using (A.12), (A.13), Order Results A and B, under Assumptions 1- 5, we obtain the following expressions

$$
\begin{aligned}
& \frac{\Delta \overline{\mathbf{Z}}^{\prime} \boldsymbol{v}_{i}}{\sqrt{T}}=\overline{\mathbf{\Gamma}} \frac{\mathbf{F}^{\prime} \boldsymbol{v}_{i}}{\sqrt{T}}+\frac{\Delta \overline{\mathbf{V}}^{\prime} \boldsymbol{v}_{i}}{\sqrt{T}}=\overline{\mathbf{\Gamma}} \frac{\mathbf{F}^{\prime} \boldsymbol{\varepsilon}_{i}}{\sigma_{i} \sqrt{T}}+O_{p}\left(\frac{1}{\sqrt{N}}\right)+O_{p}\left(\frac{\sqrt{T}}{N}\right), \\
& \frac{\boldsymbol{\tau}_{T}^{\prime} \boldsymbol{v}_{i}}{\sqrt{T}}=\frac{\boldsymbol{\tau}_{T}^{\prime} \boldsymbol{\varepsilon}_{i}}{\sigma_{i} \sqrt{T}}+O_{p}\left(\frac{1}{\sqrt{N}}\right) \\
& \frac{\overline{\mathbf{Z}}_{-1}^{\prime} \boldsymbol{v}_{i}}{T}=\overline{\mathbf{z}}_{0} \otimes\left(\frac{\boldsymbol{\tau}_{T}^{\prime} \boldsymbol{v}_{i}}{T}\right)+\overline{\boldsymbol{\Gamma}} \frac{\mathbf{S}_{f,-1}^{\prime} \boldsymbol{v}_{i}}{T}+\frac{\overline{\mathbf{S}}_{\mathbf{v},-1}^{\prime} \boldsymbol{v}_{i}}{T} \\
& =\overline{\mathbf{z}}_{0} \otimes\left(\frac{\boldsymbol{\tau}_{T}^{\prime} \boldsymbol{\varepsilon}_{i}}{\sigma_{i} T}\right)+\overline{\mathbf{\Gamma}} \frac{\mathbf{S}_{f,-1}^{\prime} \boldsymbol{\varepsilon}_{i}}{\sigma_{i} T}+O_{p}\left(\frac{1}{\sqrt{N}}\right)+O_{p}\left(\sqrt{\frac{1}{N T}}\right), \\
& \frac{\Delta \overline{\mathbf{Z}}^{\prime \circ}{ }_{i,-1}}{T^{3 / 2}}=\overline{\mathbf{\Gamma}} \frac{\mathbf{F}^{\prime \circ}{ }_{i,-1}}{T^{3 / 2}}+\frac{\Delta \overline{\mathbf{V}}^{\prime \circ}{ }_{i,-1}}{T^{3 / 2}}=\overline{\mathbf{\Gamma}} \frac{\mathbf{F}^{\prime} \mathbf{s}_{i,-1}}{\sigma_{i} T^{3 / 2}}+O_{p}\left(\frac{1}{\sqrt{T N}}\right), \\
& \frac{\boldsymbol{\tau}_{T}^{\prime}{ }_{i,-1}}{T^{3 / 2}}=\frac{\boldsymbol{\tau}_{T}^{\prime} \mathbf{s}_{i,-1}}{\sigma_{i} T^{3 / 2}}+O_{p}\left(\frac{1}{\sqrt{N}}\right), \\
& \frac{\overline{\mathbf{Z}}_{-1}^{\prime}{ }^{\circ}{ }_{i,-1}}{T^{2}}=\overline{\mathbf{z}}_{0} \otimes\left(\frac{{\boldsymbol{\boldsymbol { \tau } _ { T } ^ { \prime }}}^{\circ}{ }_{i,-1}}{T^{2}}\right)+\overline{\mathbf{\Gamma}} \frac{\mathbf{S}_{f,-1}^{\prime}{ }^{\circ} i,-1}{T^{2}}+\frac{\overline{\mathbf{S}}_{\mathbf{v},-1}^{\prime}{ }^{\circ}{ }_{i,-1}}{T^{2}} \\
& =\overline{\mathbf{z}}_{0} \otimes\left(\frac{\boldsymbol{\tau}_{T}^{\prime} \mathbf{s}_{i,-1}}{\sigma_{i} T^{2}}\right)+\overline{\mathbf{\Gamma}} \frac{\mathbf{S}_{f,-1}^{\prime} \mathbf{s}_{i,-1}}{\sigma_{i} T^{2}}+O_{p}\left(\frac{1}{\sqrt{N}}\right), \\
& \frac{\Delta \overline{\mathbf{Z}}^{\prime} \Delta \overline{\mathbf{Z}}}{T}=\overline{\mathbf{\Gamma}} \frac{\mathbf{F}^{\prime} \mathbf{F}}{T} \overline{\boldsymbol{\Gamma}}^{\prime}+\overline{\mathbf{\Gamma}} \frac{\mathbf{F}^{\prime} \Delta \overline{\mathbf{V}}}{T}+\frac{\Delta \overline{\mathbf{V}}^{\prime} \mathbf{F}}{T} \overline{\boldsymbol{\Gamma}}^{\prime}+\frac{\Delta \overline{\mathbf{V}}^{\prime} \Delta \overline{\mathbf{V}}}{T} \\
& =\overline{\mathbf{\Gamma}} \frac{\mathbf{F}^{\prime} \mathbf{F}}{T} \overline{\mathbf{\Gamma}}^{\prime}+O_{p}\left(\frac{1}{\sqrt{N T}}\right)+O_{p}\left(\frac{1}{N}\right), \\
& \frac{\boldsymbol{\tau}_{T}^{\prime} \Delta \overline{\mathbf{Z}}}{T}=\frac{\boldsymbol{\tau}_{T}^{\prime} \mathbf{F}}{T} \overline{\boldsymbol{\Gamma}}^{\prime}+\frac{\boldsymbol{\tau}_{T}^{\prime} \Delta \overline{\mathbf{V}}}{T}=\frac{\boldsymbol{\tau}_{T}^{\prime} \mathbf{F}}{T} \overline{\boldsymbol{\Gamma}}^{\prime}+O_{p}\left(\frac{1}{\sqrt{N T}}\right), \\
& \frac{\overline{\mathbf{Z}}_{-1}^{\prime} \Delta \overline{\mathbf{Z}}}{T^{3 / 2}}=\overline{\mathbf{z}}_{0} \otimes\left(\frac{\boldsymbol{\tau}_{T}^{\prime} \mathbf{F}}{T^{3 / 2}} \overline{\boldsymbol{\Gamma}}^{\prime}+\frac{\boldsymbol{\tau}_{T}^{\prime} \Delta \overline{\mathbf{V}}}{T^{3 / 2}}\right)+\overline{\mathbf{\Gamma}} \frac{\mathbf{S}_{f,-1}^{\prime} \mathbf{F}}{T^{3 / 2}} \overline{\boldsymbol{\Gamma}}^{\prime}+\overline{\mathbf{\Gamma}} \frac{\mathbf{S}_{f,-1}^{\prime} \Delta \overline{\mathbf{V}}}{T^{3 / 2}}+\frac{\overline{\mathbf{S}}_{\mathbf{v},-1}^{\prime} \mathbf{F}}{T^{3 / 2}} \overline{\boldsymbol{\Gamma}}^{\prime}+\frac{\overline{\mathbf{S}}_{\mathbf{v},-1}^{\prime} \Delta \overline{\mathbf{V}}}{T^{3 / 2}} \\
& =\overline{\mathbf{z}}_{0} \otimes\left(\frac{\boldsymbol{\tau}_{T}^{\prime} \mathbf{F}}{T^{3 / 2}} \overline{\mathbf{\Gamma}}^{\prime}\right)+\overline{\mathbf{\Gamma}} \frac{\mathbf{S}_{f,-1}^{\prime} \mathbf{F}}{T^{3 / 2}} \overline{\mathbf{\Gamma}}^{\prime}+O_{p}\left(\frac{1}{\sqrt{N T}}\right), \\
& \frac{\overline{\mathbf{Z}}_{-1}^{\prime} \boldsymbol{\tau}_{T}}{T^{3 / 2}}=\frac{\overline{\mathbf{z}}_{0}}{\sqrt{T}}+\overline{\mathbf{\Gamma}} \frac{\mathbf{S}_{f,-1}^{\prime} \boldsymbol{\tau}_{T}}{T^{3 / 2}}+\frac{\overline{\mathbf{S}}_{\mathbf{v},-1}^{\prime} \boldsymbol{\tau}_{T}}{T^{3 / 2}}=\frac{\overline{\mathbf{z}}_{0}}{\sqrt{T}}+\overline{\boldsymbol{\Gamma}} \frac{\mathbf{S}_{f,-1}^{\prime} \boldsymbol{\tau}_{T}}{T^{3 / 2}}+O_{p}\left(\frac{1}{\sqrt{N}}\right) \\
& \frac{\overline{\mathbf{Z}}_{-1}^{\prime} \overline{\mathbf{Z}}_{-1}}{T^{2}}=\frac{\overline{\mathbf{z}}_{0} \overline{\mathbf{z}}_{0}^{\prime}}{T}+\overline{\mathbf{\Gamma}} \frac{\mathbf{S}_{f,-1}^{\prime} \mathbf{S}_{f,-1}}{T^{2}} \overline{\boldsymbol{\Gamma}}^{\prime}+\frac{\overline{\mathbf{S}}_{\mathbf{v},-1}^{\prime} \overline{\mathbf{S}}_{\mathbf{v},-1}}{T^{2}}+\frac{\overline{\mathbf{S}}_{\mathbf{v},-1}^{\prime} \mathbf{S}_{f,-1}}{T^{2}} \overline{\boldsymbol{\Gamma}}^{\prime}+\overline{\mathbf{\Gamma}} \frac{\mathbf{S}_{f,-1}^{\prime} \overline{\mathbf{S}}_{\mathbf{v},-1}^{\prime}}{T^{2}} \\
& +\overline{\mathbf{z}}_{0} \otimes \frac{\boldsymbol{\tau}_{T}^{\prime} \mathbf{S}_{f,-1}}{T^{2}} \overline{\boldsymbol{\Gamma}}^{\prime}+\overline{\mathbf{\Gamma}} \frac{\mathbf{S}_{f,-1}^{\prime} \boldsymbol{\tau}_{T}}{T^{2}} \otimes \overline{\mathbf{z}}_{0}^{\prime} \\
& +\overline{\mathbf{z}}_{0} \otimes \frac{\boldsymbol{\tau}_{T}^{\prime} \overline{\mathbf{S}}_{\mathbf{v},-1}}{T^{2}}+\frac{\overline{\mathbf{S}}_{\mathbf{v},-1}^{\prime} \boldsymbol{\tau}_{T}}{T^{2}} \otimes \overline{\mathbf{z}}_{0}^{\prime} \\
& =\frac{\overline{\mathbf{z}}_{0} \overline{\mathbf{z}}_{0}^{\prime}}{T}+\overline{\mathbf{z}}_{0} \otimes \frac{\boldsymbol{\tau}_{T}^{\prime} \mathbf{S}_{f,-1}}{T^{2}} \overline{\boldsymbol{\Gamma}}^{\prime}+\overline{\boldsymbol{\Gamma}} \frac{\mathbf{S}_{f,-1}^{\prime} \boldsymbol{\tau}_{T}}{T^{2}} \otimes \overline{\mathbf{z}}_{0}^{\prime} \\
& +\overline{\boldsymbol{\Gamma}} \frac{\mathbf{S}_{f,-1}^{\prime} \mathbf{S}_{f,-1}}{T^{2}} \overline{\boldsymbol{\Gamma}}^{\prime}+O_{p}\left(\frac{1}{\sqrt{N T}}\right)+O_{p}\left(\frac{1}{\sqrt{N}}\right), \\
& \frac{{ }_{i,-1}^{\circ} \boldsymbol{v}_{i}}{T^{3 / 2}}=\frac{\mathbf{s}_{i,-1}^{\prime} \varepsilon_{i}}{\sigma_{i}^{2} T^{3 / 2}}+O_{p}\left(\frac{1}{\sqrt{N T}}\right), \\
& \frac{{ }^{\circ},-1{ }^{\circ} i,-1}{T^{2}}=\frac{\mathbf{s}_{i,-1}^{\prime} \mathbf{s}_{i,-1}}{\sigma_{i}^{2} T^{2}}+O_{p}\left(\frac{1}{\sqrt{N}}\right) \text {. }
\end{aligned}
$$




\section{A.3 Proof of Theorem 2.1:}

\section{A.3.1 $\quad$ T fixed and $N \rightarrow \infty$}

Recall equation (27) that can be written as

$$
t_{i}(N, T)=\frac{\frac{\boldsymbol{v}_{i}^{\prime} \overline{\mathbf{M}}_{i,-1}^{\mathfrak{s}_{i,-1}}}{T}}{\left(\frac{\boldsymbol{v}_{i}^{\prime} \overline{\mathbf{M}}_{i} \boldsymbol{v}_{i}}{T-2 k-4}\right)^{1 / 2}\left(\frac{\overline{\mathfrak{s}}_{i,-1}^{\prime} \overline{\mathbf{M}}_{\mathfrak{s}_{i,-1}}}{T^{2}}\right)^{1 / 2}} .
$$

Expanding $\boldsymbol{v}_{i}^{\prime} \overline{\mathbf{M}}_{i,-1}^{\circ} / T$ in (27) gives

$$
\frac{\boldsymbol{v}_{i}^{\prime} \overline{\mathbf{M}}_{i,-1}^{\circ}}{T}=\frac{\boldsymbol{v}_{i}^{\prime \circ}{ }_{i,-1}}{T}-\left(\boldsymbol{v}_{i}^{\prime} \overline{\mathbf{W}} \mathbf{B}\right)\left(\mathbf{B} \overline{\mathbf{W}}^{\prime} \overline{\mathbf{W}} \mathbf{B}\right)^{-1}\left(\frac{\mathbf{B} \overline{\mathbf{W}}^{\prime \circ}{ }_{i,-1}}{T}\right),
$$

where

$$
\begin{gathered}
\mathbf{B}=\left[\begin{array}{cc}
\frac{1}{\sqrt{T}} \mathbf{I}_{k+2} & \mathbf{0} \\
\mathbf{0} & \frac{1}{T} \mathbf{I}_{k+1}
\end{array}\right] \\
\mathbf{B} \overline{\mathbf{W}}^{\prime} \boldsymbol{v}_{i}=\left(\begin{array}{c}
\Delta \overline{\mathbf{Z}}^{\prime} \boldsymbol{v}_{i} / \sqrt{T} \\
\boldsymbol{\tau}_{T}^{\prime} \boldsymbol{v}_{i} / \sqrt{T} \\
\overline{\mathbf{Z}}_{-1}^{\prime} \boldsymbol{v}_{i} / T
\end{array}\right), \frac{\mathbf{B} \overline{\mathbf{W}}^{\prime \circ}{ }_{i,-1}}{T}=\left(\begin{array}{c}
\Delta \overline{\mathbf{Z}}^{\prime \circ}{ }_{i,-1} / T^{3 / 2} \\
\boldsymbol{\tau}_{T}^{\prime}{ }^{\circ}{ }_{i,-1} / T^{3 / 2} \\
\overline{\mathbf{Z}}_{-1}^{\prime}{ }_{i,-1} / T^{2}
\end{array}\right),
\end{gathered}
$$

and

$$
\mathbf{B} \overline{\mathbf{W}}^{\prime} \overline{\mathbf{W}} \mathbf{B}=\left(\begin{array}{ccc}
\frac{\Delta \overline{\mathbf{Z}}^{\prime} \Delta \overline{\mathbf{Z}}}{T} & \frac{\Delta \overline{\mathbf{X}}^{\prime} \boldsymbol{\tau}_{T}}{T} & \frac{\Delta \overline{\mathbf{Z}}^{\prime} \overline{\mathbf{Z}}_{-1}}{T^{3 / 2}} \\
\frac{\boldsymbol{\tau}_{T}^{\prime} \Delta \overline{\mathbf{Z}}}{\overline{\mathbf{Z}}_{T}^{\prime}} & \frac{\boldsymbol{\tau}_{T}^{\prime} \boldsymbol{\tau}_{T}}{T} & \frac{\boldsymbol{\tau}_{T}^{T} \overline{\mathbf{Z}}_{-1}}{\overline{\mathbf{Z}}_{-1}^{\prime} \Delta \overline{\mathbf{Z}}} \\
\frac{\overline{\mathbf{Z}}_{-1}^{\prime} \boldsymbol{\tau}_{T}}{T^{3 / 2}} & \frac{\overline{\mathbf{z}}_{-1}^{T^{3} \overline{\mathbf{Z}}_{-1}^{2}}}{T^{3 / 2}} & \frac{T^{2}}{T^{2}}
\end{array}\right) .
$$

Next, note that $\overline{\mathbf{M}}_{i} \boldsymbol{v}_{i}$ are the residuals from the regression of $\boldsymbol{v}_{i}$ on $\overline{\mathbf{W}}_{i}=\left(\overline{\mathbf{W}}, \mathbf{y}_{i,-1}\right)$, but from equation (24) $\mathbf{y}_{i,-1}$ has components $\left(\overline{\mathbf{Z}}_{-1}, \boldsymbol{\tau}_{T},{ }_{i,-1}\right)$. As $\left(\boldsymbol{\tau}_{T}, \overline{\mathbf{Z}}_{-1}\right) \subset \mathbf{\mathbf { W }}$, but ${ }^{\circ}{ }_{i,-1}$ is not contained in $\overline{\mathbf{W}}$, by regression theory

$$
\overline{\mathbf{M}}_{i} \boldsymbol{v}_{i}=\overline{\mathbf{M}}_{i}^{*} \boldsymbol{v}_{i}
$$

where

with $\overline{\mathbf{H}}_{i}=\left(\overline{\mathbf{W}},{ }_{i,-1}\right)$. Thus

$$
\overline{\mathbf{M}}_{i}^{*}=\mathbf{I}-\overline{\mathbf{H}}_{i}\left(\overline{\mathbf{H}}_{i}^{\prime} \overline{\mathbf{H}}_{i}\right) \overline{\mathbf{H}}_{i}^{\prime},
$$

$$
\boldsymbol{v}_{i} \overline{\mathbf{M}}_{i}^{*} \boldsymbol{v}_{i}=\boldsymbol{v}_{i}^{\prime} \boldsymbol{v}_{i}-\left(\boldsymbol{v}_{i}^{\prime} \overline{\mathbf{H}}_{i} \mathbf{E}^{\prime}\right)\left(\mathbf{E} \overline{\mathbf{H}}_{i}^{\prime} \overline{\mathbf{H}}_{i} \mathbf{E}^{\prime}\right)^{-1}\left(\mathbf{E} \overline{\mathbf{H}}_{i}^{\prime} \boldsymbol{v}_{i}\right),
$$

where

$$
\mathbf{E}=\left(\begin{array}{cc}
\mathbf{B} & \mathbf{0} \\
\mathbf{0} & T^{-1}
\end{array}\right)
$$

Also,

$$
\mathbf{E} \overline{\mathbf{H}}_{i}^{\prime} \boldsymbol{v}_{i}=\left(\begin{array}{c}
\mathbf{B} \overline{\mathbf{W}}^{\prime} \boldsymbol{v}_{i} \\
{ }_{i,-1}^{\prime} \boldsymbol{v}_{i} / T
\end{array}\right), \mathbf{E} \overline{\mathbf{H}}_{i}^{\prime} \overline{\mathbf{H}}_{i} \mathbf{E}^{\prime}=\left(\begin{array}{cc}
\mathbf{B} \overline{\mathbf{W}}^{\prime} \overline{\mathbf{W}} \mathbf{B} & \mathbf{B} \overline{\mathbf{W}}^{\prime \circ}{ }_{i,-1} / T \\
{ }_{i,-1}^{\prime} \mathbf{\mathbf { W }} \mathbf{B} / T & \stackrel{{ }^{\prime},}{i,-1}{ }_{i,-1} / T^{2}
\end{array}\right) .
$$

Under Assumptions 1-5, using the order results in (A.2) and assuming $\overline{\mathbf{z}}_{0}=\mathbf{0}$ or re-defining $\mathbf{z}_{i t}$ as the deviation from $\overline{\mathbf{z}}_{0}$, as $N \rightarrow \infty$ with $T$ fixed,

$$
\mathbf{B} \overline{\mathbf{W}}^{\prime} \boldsymbol{v}_{i} \stackrel{N}{\rightarrow} \boldsymbol{\Xi}_{i T}, \frac{\mathbf{B} \overline{\mathbf{W}}^{\prime \circ}{ }_{i,-1}}{T} \stackrel{N}{\rightarrow} \boldsymbol{\Xi}_{i T}, \mathbf{B} \overline{\mathbf{W}}^{\prime} \overline{\mathbf{W}} \mathbf{B} \stackrel{N}{\rightarrow} \boldsymbol{\Xi} \boldsymbol{\Upsilon}_{\varphi T} \boldsymbol{\Xi}^{\prime}
$$

where

$$
\begin{gathered}
\boldsymbol{\Xi}=\left(\begin{array}{ccc}
\boldsymbol{\Gamma} & \mathbf{0} & \mathbf{0} \\
\mathbf{0} & 1 & \mathbf{0} \\
\mathbf{0} & \mathbf{0} & \boldsymbol{\Gamma}
\end{array}\right)=\operatorname{plim}_{N \rightarrow \infty}\left(\begin{array}{ccc}
\overline{\boldsymbol{\Gamma}} & \mathbf{0} & \mathbf{0} \\
\mathbf{0} & 1 & \mathbf{0} \\
\mathbf{0} & \mathbf{0} & \overline{\boldsymbol{\Gamma}}
\end{array}\right) . \\
\underset{(2 m+1 \times 1)}{\mathbf{q}_{i T}}=\left(\begin{array}{c}
\frac{\mathbf{F}^{\prime} \varepsilon_{i}}{\sigma_{i} \sqrt{T}} \\
\frac{\boldsymbol{\tau}_{T}^{\prime} \boldsymbol{\varepsilon}_{i}}{\sigma_{i} \sqrt{T}} \\
\mathbf{s}_{f,-1}^{\prime} \boldsymbol{\varepsilon}_{i} \\
\sigma_{i} T
\end{array}\right), \underset{(2 m+1 \times 1)}{\mathbf{h}_{i T}}=\left(\begin{array}{c}
\frac{\Delta \boldsymbol{\Phi}^{\prime} \mathbf{s}_{i,-1}}{\sigma_{i} T^{3 / 2}} \\
\frac{\boldsymbol{\tau}_{T}^{\prime} \mathbf{s}_{i,-1}}{\sigma_{i} T^{3 / 2}} \\
\frac{\mathbf{s}_{f,-1}^{\prime} \mathbf{s}_{i,-1}}{\sigma_{i} T^{2}}
\end{array}\right),
\end{gathered}
$$




$$
\begin{aligned}
\mathbf{E}_{i}^{\prime} \boldsymbol{v}_{i} \stackrel{N}{\rightarrow} \boldsymbol{\Xi}_{*} \mathbf{d}_{i T}, \text { with } \mathbf{g}_{i T} & =\left(\begin{array}{c}
\mathbf{q}_{i T} \\
\frac{\mathbf{s}_{i,-}^{\prime} \varepsilon_{i}}{\sigma_{i}^{2} T}
\end{array}\right), \\
\mathbf{E} \overline{\mathbf{H}}_{i}^{\prime} \overline{\mathbf{H}}_{i} \mathbf{E}^{\prime} \stackrel{N}{\rightarrow} \boldsymbol{\Xi}_{*} \mathbf{Q}_{i T} \boldsymbol{\Xi}_{*}^{\prime}, \text { with } \mathbf{Q}_{i T} & =\left(\begin{array}{cc}
\mathbf{\Upsilon}_{f T} & \mathbf{h}_{i T} \\
\mathbf{h}_{i T}^{\prime} & \frac{\mathbf{s}_{i,-1}^{\prime} \mathbf{s}_{i,-1}}{\sigma_{i}^{2} T^{2}}
\end{array}\right),
\end{aligned}
$$

and

$$
\begin{gathered}
\boldsymbol{\Xi}_{*}=\left(\begin{array}{cc}
\mathbf{\Xi} & \mathbf{0} \\
\mathbf{0} & 1
\end{array}\right), \\
\boldsymbol{\Upsilon}_{f T}=\left(\begin{array}{ccc}
\frac{\mathbf{F}^{\prime} \mathbf{F}}{T} & \frac{\mathbf{F}^{\prime} \boldsymbol{\tau}_{T}}{T} & \frac{\mathbf{F}^{\prime} \mathbf{S}_{f,-1}}{T^{3 / 2}} \\
\frac{\boldsymbol{\tau}_{T}^{\prime} \mathbf{F}}{T} & 1 & \boldsymbol{\tau}_{T}^{\prime} \mathbf{S}_{f,-1} \\
\frac{\mathbf{S}_{f,-1}^{\prime} \mathbf{F}}{T^{3 / 2}} & \frac{\mathbf{S}_{f,-1}^{\prime} \boldsymbol{\tau}_{T}}{T^{3 / 2}} & \frac{\mathbf{S}_{f,-1}^{\prime} \mathbf{S}_{f,-1}}{T^{2}}
\end{array}\right) .
\end{gathered}
$$

The $(2 k+4) \times(2 k+4)$ matrix $\boldsymbol{\Xi} \Upsilon_{f T} \boldsymbol{\Xi}^{\prime}$ has rank $2 m+1 \leq 2 k+4$ due to rank condition (9), and thus under Assumptions 1-5 we obtain

$$
t_{i}(N, T) \stackrel{N}{\Longrightarrow} \frac{\frac{\boldsymbol{\varepsilon}_{i}^{\prime} \mathbf{s}_{i,-1}}{\sigma_{i}^{2} T}-\mathbf{q}_{i T}^{\prime} \boldsymbol{\Xi}^{\prime}\left(\boldsymbol{\Xi} \mathbf{\Upsilon}_{f T} \boldsymbol{\Xi}^{\prime}\right)^{-} \boldsymbol{\Xi} \mathbf{h}_{i T}}{\left(\frac{\boldsymbol{\varepsilon}_{\boldsymbol{\varepsilon}}^{\prime} \boldsymbol{\varepsilon}_{i}}{\sigma_{i}^{2}(T-2 k-4)}-\frac{\mathbf{g}_{i T}^{\prime} \boldsymbol{\Xi}_{*}^{\prime}\left(\boldsymbol{\Xi}_{*} \mathbf{Q}_{i T} \boldsymbol{\Xi}_{*}^{\prime}\right)^{-} \boldsymbol{\Xi}_{*} \mathbf{g}_{i T}}{(T-2 k-4)}\right)^{1 / 2}\left(\frac{\mathbf{s}_{i,-1}^{\prime} \mathbf{s}_{i,-1}}{\sigma_{i}^{2} T^{2}}-\mathbf{h}_{i T}^{\prime} \boldsymbol{\Xi}^{\prime}\left(\boldsymbol{\Xi} \mathbf{\Upsilon}_{f T} \boldsymbol{\Xi}^{\prime}\right)^{-} \boldsymbol{\Xi} \mathbf{h}_{i T}\right)^{1 / 2}},
$$

and as

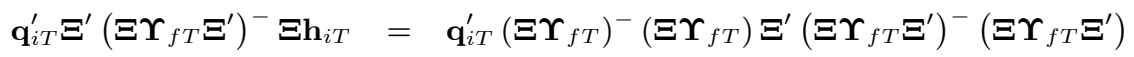

$$
\begin{aligned}
& \left(\boldsymbol{\Xi} \Upsilon_{f T} \boldsymbol{\Xi}^{\prime}\right)^{-} \boldsymbol{\Xi}\left(\boldsymbol{\Upsilon}_{f T} \boldsymbol{\Xi}^{\prime}\right)\left(\boldsymbol{\Upsilon}_{f T} \boldsymbol{\Xi}^{\prime}\right)^{-} \mathbf{h}_{i T} \\
& =\mathbf{q}_{i T}^{\prime}\left(\boldsymbol{\Xi} \Upsilon_{f T}\right)^{-}\left(\boldsymbol{\Xi} \Upsilon_{f T} \boldsymbol{\Xi}^{\prime}\right)\left(\boldsymbol{\Upsilon}_{f T} \boldsymbol{\Xi}^{\prime}\right)^{-} \mathbf{h}_{i T} \\
& =\mathbf{q}_{i T}^{\prime} \mathbf{\Upsilon}_{f T}^{-1} \mathbf{h}_{i T} \text {, }
\end{aligned}
$$

where the last line follows using the results of generalised inverse (Magnus and Neudecker, 1999; Miscellaneous Exercises 6, p.38) and similarly for $\mathbf{g}_{i T}^{\prime} \boldsymbol{\Xi}_{*}^{\prime}\left(\boldsymbol{\Xi}_{*} \mathbf{Q}_{i T} \boldsymbol{\Xi}_{*}^{\prime}\right)^{-} \boldsymbol{\Xi}_{*} \mathbf{g}_{i T}$, it follows that for $\operatorname{rank}(\bar{\Gamma})=m \leq k+1$,

$$
t_{i}(N, T) \stackrel{N}{\Longrightarrow} \frac{\frac{\boldsymbol{\varepsilon}_{i}^{\prime} \mathbf{s}_{i,-1}}{\sigma_{i}^{T} T}-\mathbf{q}_{i T}^{\prime} \mathbf{\Upsilon}_{f T}^{-1} \mathbf{h}_{i T}}{\left(\frac{\boldsymbol{\varepsilon}_{i}^{\prime} \varepsilon_{i}}{\sigma_{i}^{2}(T-2 k-4)}-\frac{\mathbf{g}_{i T}^{\prime} \mathbf{Q}_{i T}^{-1} \mathbf{g}_{i T}}{(T-2 k-4)}\right)^{1 / 2}\left(\frac{\mathbf{s}_{i,-1}^{\prime} \mathbf{s}_{i,-1}}{\sigma_{i}^{2} T^{2}}-\mathbf{h}_{i T}^{\prime} \mathbf{\Upsilon}_{f T}^{-1} \mathbf{h}_{i T}\right)^{1 / 2}},
$$

which is free of nuisance parameters.

\section{A.4 Proof of Theorem 2.2:}

\section{A.4.1 Sequential Asymptotics: $N \rightarrow \infty$ then $T \rightarrow \infty$}

Using Proposition 17.1 and 18.1 of Hamilton (1994; p.486, p.547-8), under Assumptions 1-5 we have

$$
\begin{gathered}
\mathbf{q}_{i T} \stackrel{T}{\Longrightarrow} \boldsymbol{\vartheta}_{i f}=\left(\begin{array}{c}
\boldsymbol{\Lambda}_{f} \mathbf{W}_{f, i}(1) \\
\boldsymbol{\Lambda}_{f *} \boldsymbol{\omega}_{i f}
\end{array}\right), \mathbf{h}_{i T} \stackrel{T}{\Longrightarrow} \boldsymbol{\kappa}_{i f}=\left(\begin{array}{c}
\mathbf{0} \\
\boldsymbol{\Lambda}_{f^{*}} \boldsymbol{\pi}_{i f}
\end{array}\right) \\
\boldsymbol{\Upsilon}_{f T} \stackrel{T}{\Longrightarrow} \boldsymbol{\Upsilon}_{f}=\left(\begin{array}{cc}
\boldsymbol{\Sigma}_{f 0} & \mathbf{0} \\
\mathbf{0} & \boldsymbol{\Lambda}_{f *} \mathbf{G}_{f} \boldsymbol{\Lambda}_{f^{*}}^{\prime}
\end{array}\right),
\end{gathered}
$$

where

$$
\begin{gathered}
\boldsymbol{\Lambda}_{f^{*}}=\left(\begin{array}{cc}
1 & \mathbf{0} \\
\mathbf{0} & \mathbf{\Lambda}_{f}
\end{array}\right), \\
\boldsymbol{\omega}_{i f}=\left(\begin{array}{c}
W_{i}(1) \\
\int_{0}^{1}\left[\mathbf{W}_{f}(r)\right] d W_{i}(r)
\end{array}\right), \boldsymbol{\pi}_{i f}=\left(\begin{array}{c}
\int_{0}^{1} W_{i}(r) d r \\
\int_{0}^{1}\left[\mathbf{W}_{f}(r)\right] W_{i}(r) d r
\end{array}\right),
\end{gathered}
$$


and

$$
\mathbf{G}_{f}=\left(\begin{array}{cc}
1 & \int_{0}^{1}\left[\mathbf{W}_{f}(r)\right]^{\prime} d r \\
\int_{0}^{1}\left[\mathbf{W}_{f}(r)\right] d r & \int_{0}^{1}\left[\mathbf{W}_{f}(r)\right]\left[\mathbf{W}_{f}(r)\right]^{\prime} d r
\end{array}\right) .
$$

From the Order Results in (A.2) we have that

$$
\begin{aligned}
t_{i}(N, T) \stackrel{(N, T)}{\Longrightarrow} \frac{\int_{0}^{1} W_{i}(r) d W_{i}(r)-\boldsymbol{\omega}_{i f}^{\prime} \boldsymbol{\Lambda}_{f *}^{\prime}\left(\boldsymbol{\Lambda}_{f} \mathbf{G}_{f} \boldsymbol{\Lambda}_{f *}^{\prime}\right)^{-} \boldsymbol{\Lambda}_{f *} \boldsymbol{\pi}_{i f}}{\left(\int_{0}^{1} W_{i}^{2}(r) d r-\boldsymbol{\pi}_{i f}^{\prime} \boldsymbol{\Lambda}_{f *}^{\prime}\left(\boldsymbol{\Lambda}_{f *} \mathbf{G}_{f} \boldsymbol{\Lambda}_{f^{*}}^{\prime}\right)-\boldsymbol{\Lambda}_{f *} \boldsymbol{\pi}_{i f}\right)^{1 / 2}} \\
=\frac{\int_{0}^{1} W_{i}(r) d W_{i}(r)-\boldsymbol{\omega}_{i f}^{\prime} \mathbf{G}_{f}^{-1} \boldsymbol{\pi}_{i f}}{\left(\int_{0}^{1} W_{i}^{2}(r) d r-\boldsymbol{\pi}_{i f}^{\prime} \mathbf{G}_{f}^{-1} \boldsymbol{\pi}_{i f}\right)^{1 / 2}} .
\end{aligned}
$$

\section{A.4.2 Joint Asymptotics}

From Order Results in (A.2) it follows that

$$
t_{i}(N, T) \stackrel{(N, T)_{j}}{\Longrightarrow} \frac{\int_{0}^{1} W_{i}(r) d W_{i}(r)-\boldsymbol{\omega}_{i f}^{\prime} \mathbf{G}_{f}^{-1} \boldsymbol{\pi}_{i f}}{\left(\int_{0}^{1} W_{i}^{2}(r) d r-\boldsymbol{\pi}_{i f}^{\prime} \mathbf{G}_{f}^{-1} \boldsymbol{\pi}_{i f}\right)^{1 / 2}}
$$

as $T$ and $N$ go to infinity so long as $\sqrt{T} / N \rightarrow 0$. This condition is satisfied as $T / N \rightarrow \delta$, where $\delta$ is a fixed finite non-zero positive constant.

\section{A.5 Proof of Theorem 2.3}

Recall equation (48) that can written as

$$
t_{i}(N, T)=\frac{\frac{\boldsymbol{v}_{i}^{\prime} \overline{\mathbf{M}}_{i 1 \mathfrak{s}^{\circ}}}{T}}{\left(\frac{\boldsymbol{v}_{i \zeta,-1}^{\prime} \overline{\mathbf{M}}_{i 1, p} \boldsymbol{v}_{i}}{T-3 k-6}\right)^{1 / 2}\left(\frac{\mathfrak{s}_{i \zeta,-1}^{\prime} \overline{\mathbf{M}}_{i 11^{\mathfrak{s}}}{ }_{i \zeta,-1}}{T^{2}}\right)^{1 / 2}},
$$

where $\boldsymbol{v}_{i}=\left[\boldsymbol{\eta}_{i}-\left(\Delta \overline{\mathbf{V}}-\boldsymbol{\theta} \Delta \overline{\mathbf{V}}_{-1}\right) \boldsymbol{\delta}_{i}\right] / \sigma_{i \eta}$ and ${ }^{\circ}{ }_{i \zeta,-1}=\left(\mathbf{s}_{i \zeta,-1}-\overline{\mathbf{S}}_{\mathbf{v},-1} \boldsymbol{\delta}_{i}\right) / \sigma_{i \eta}$.

Expanding $\boldsymbol{v}_{i}^{\prime} \overline{\mathbf{M}}_{i 1}{ }_{i \zeta,-1} / T$ (48) gives

$$
\frac{\boldsymbol{v}_{i}^{\prime} \overline{\mathbf{M}}_{i 1}{ }^{\circ}{ }_{i \zeta,-1}}{T}=\frac{\boldsymbol{v}_{i}^{\prime \circ}{ }_{i \zeta,-1}}{T}-\left(\boldsymbol{v}_{i}^{\prime} \overline{\mathbf{W}}_{i 1} \mathbf{B}_{1}\right)\left(\mathbf{B}_{1} \overline{\mathbf{W}}_{i 1}^{\prime} \overline{\mathbf{W}}_{i 1} \mathbf{B}_{1}\right)^{-1}\left(\frac{\mathbf{B}_{1} \overline{\mathbf{W}}_{i 1}^{\prime}{ }^{\circ}{ }_{i \zeta,-1}}{T}\right),
$$

where $\overline{\mathbf{W}}_{i 1}=\left(\Delta \mathbf{y}_{i,-1}, \Delta \overline{\mathbf{Z}}, \Delta \overline{\mathbf{Z}}_{-1}, \boldsymbol{\tau}_{T}, \overline{\mathbf{Z}}_{-1}\right)$ and

$$
\mathbf{B}_{1}=\left[\begin{array}{cc}
\frac{1}{\sqrt{T}} \mathbf{I}_{2 k+4} & \mathbf{0} \\
\mathbf{0} & \frac{1}{T} \mathbf{I}_{k+1}
\end{array}\right] .
$$

Using the results set out above, together with the results in Propositions 17.3 and 18.1 of Hamilton (1994), for example, as $N$ and $T \rightarrow \infty$ (sequentially and) jointly such that $\sqrt{T} / N \rightarrow 0$, we obtain

$$
\mathbf{B}_{1} \overline{\mathbf{W}}_{i 1}^{\prime} \boldsymbol{v}_{i} \stackrel{(N, T)_{j}}{\Longrightarrow} \Theta_{1} \boldsymbol{\vartheta}_{i f 1},
$$

where

$$
\underset{3(k+1)+2 \times 3 m+2}{\Theta_{1}}=\left(\begin{array}{ccccc}
1 & \mathbf{0} & \mathbf{0} & \mathbf{0} & \mathbf{0} \\
\mathbf{0} & \boldsymbol{\Gamma} & \mathbf{0} & \mathbf{0} & \mathbf{0} \\
\mathbf{0} & \mathbf{0} & \boldsymbol{\Gamma} & \mathbf{0} & \mathbf{0} \\
\mathbf{0} & \mathbf{0} & \mathbf{0} & 1 & \mathbf{0} \\
\mathbf{0} & \mathbf{0} & \mathbf{0} & \mathbf{0} & \boldsymbol{\Gamma}
\end{array}\right), \boldsymbol{\vartheta}_{i f 1}=\left(\begin{array}{c}
\gamma_{i}^{\prime} \boldsymbol{\Lambda}_{f} \mathbf{W}_{f, i}(1)+\sqrt{\frac{\sigma_{i \eta}^{2}}{1-\theta^{2}}} W_{i}(1) \\
\boldsymbol{\Lambda}_{f} \mathbf{W}_{f, i}(1) \\
\boldsymbol{\Lambda}_{f} \mathbf{W}_{f, i}(1) \\
\boldsymbol{\Lambda}_{f *} \boldsymbol{\omega}_{i f}
\end{array}\right)
$$


$\boldsymbol{\Gamma}=\operatorname{plim}_{N \rightarrow \infty} \overline{\boldsymbol{\Gamma}}, \boldsymbol{\Lambda}_{f}, \boldsymbol{\Lambda}_{f} *$ and $\boldsymbol{\omega}_{i f}$ are defined in Assumption 2, (A.38) and (A.39), respectively,

$$
\frac{\mathbf{B}_{1} \overline{\mathbf{W}}_{i 1}^{\prime}{ }^{\circ} i \zeta,-1}{T} \stackrel{(N, T)_{j}}{\Longrightarrow} \Theta_{1} \boldsymbol{\kappa}_{i f 1}, \boldsymbol{\kappa}_{i f 1}=\left(\begin{array}{c}
\mathbf{0}_{2 m+1} \\
\frac{1}{1-\theta} \boldsymbol{\Lambda}_{f *} \boldsymbol{\pi}_{i f}
\end{array}\right),
$$

$\boldsymbol{\pi}_{i f}$ is defined in (A.39),

$$
\mathbf{B}_{1} \overline{\mathbf{W}}_{i 1}^{\prime} \overline{\mathbf{W}}_{i 1} \mathbf{B}_{1} \stackrel{(N, T)_{j}}{\Longrightarrow} \Theta_{1} \boldsymbol{\Upsilon}_{i f 1} \Theta_{1}^{\prime}, \boldsymbol{\Upsilon}_{i f 1}=\left(\begin{array}{cc}
\varkappa_{i f 1} & \mathbf{0}_{2 m+1 \times m+1} \\
\mathbf{0}_{2 m+1 \times m+1}^{\prime} & \boldsymbol{\Lambda}_{f^{*}} \mathbf{G}_{f} \boldsymbol{\Lambda}_{f^{*}}^{\prime}
\end{array}\right)
$$

where

$$
\varkappa_{i f 1}=\left(\begin{array}{ccc}
\gamma_{i}^{\prime}\left[2 \boldsymbol{\Sigma}_{f 0}-\left(\boldsymbol{\Sigma}_{f 1}+\boldsymbol{\Sigma}_{f 1}^{\prime}\right)\right] \gamma_{i}+\frac{\boldsymbol{\sigma}_{\eta i}^{2}}{1-\theta^{2}} & \gamma_{i}^{\prime}\left[2 \boldsymbol{\Sigma}_{f 1}^{\prime}-\left(\boldsymbol{\Sigma}_{f 0}+\boldsymbol{\Sigma}_{f 2}^{\prime}\right)\right] & \gamma_{i}^{\prime}\left[2 \boldsymbol{\Sigma}_{f 0}-\left(\boldsymbol{\Sigma}_{f 1}+\boldsymbol{\Sigma}_{f 1}^{\prime}\right)\right] \\
{\left[2 \boldsymbol{\Sigma}_{f 1}-\left(\boldsymbol{\Sigma}_{f 0}+\boldsymbol{\Sigma}_{f 2}\right)\right] \gamma_{i}} & {\left[2 \boldsymbol{\Sigma}_{f 0}-\left(\boldsymbol{\Sigma}_{f 1}+\boldsymbol{\Sigma}_{f 1}^{\prime}\right)\right]} & {\left[2 \boldsymbol{\Sigma}_{f 1}-\left(\boldsymbol{\Sigma}_{f 0}+\boldsymbol{\Sigma}_{f 2}\right)\right]} \\
{\left[2 \boldsymbol{\Sigma}_{f 0}-\left(\boldsymbol{\Sigma}_{f 1}+\boldsymbol{\Sigma}_{f 1}^{\prime}\right)\right] \gamma_{i}} & {\left[2 \boldsymbol{\Sigma}_{f 1}^{\prime}-\left(\boldsymbol{\Sigma}_{f 0}+\boldsymbol{\Sigma}_{f 2}^{\prime}\right)\right]} & {\left[2 \boldsymbol{\Sigma}_{f 0}-\left(\boldsymbol{\Sigma}_{f 1}+\boldsymbol{\Sigma}_{f 1}^{\prime}\right)\right]}
\end{array}\right),
$$

$\mathbf{G}_{f}$ is defined by (A.40), and

$$
\frac{\boldsymbol{v}_{i}^{\prime \circ} i \zeta,-1}{T} \stackrel{(N, T)_{j}}{=} \frac{1}{1-\theta} \int_{0}^{1} W_{i}(r) d W_{i}(r)
$$

For the term $\boldsymbol{v}_{i}^{\prime} \overline{\mathbf{M}}_{i 1, p} \boldsymbol{v}_{i}$, following a similar reasoning as in the uncorrelated case we can write

$$
\boldsymbol{v}_{i}^{\prime} \overline{\mathbf{M}}_{i 1, p} \boldsymbol{v}_{i}=\boldsymbol{v}_{i}^{\prime} \boldsymbol{v}_{i}-\left(\boldsymbol{v}_{i}^{\prime} \overline{\mathbf{H}}_{i 1} \mathbf{E}_{1}^{\prime}\right)\left(\mathbf{E}_{1} \overline{\mathbf{H}}_{i 1}^{\prime} \overline{\mathbf{H}}_{i 1} \mathbf{E}_{1}^{\prime}\right)^{-1}\left(\mathbf{E}_{1} \overline{\mathbf{H}}_{i 1}^{\prime} \boldsymbol{v}_{i}\right),
$$

where $\overline{\mathbf{H}}_{i 1}=\left(\overline{\mathbf{W}}_{i 1},{ }_{i \zeta,-1}\right)$ and

$$
\mathbf{E}_{1}=\left(\begin{array}{cc}
\mathbf{B}_{1} & \mathbf{0} \\
\mathbf{0} & T^{-1}
\end{array}\right)
$$

so that

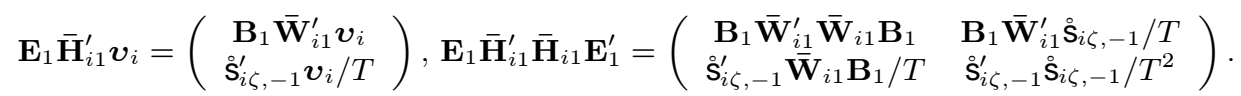

It is easily seen that $\boldsymbol{v}_{i}^{\prime} \overline{\mathbf{M}}_{i 1, p} \boldsymbol{v}_{i} /(T-3 k-6) \stackrel{(N, T)_{j}}{\Longrightarrow} 1$ using the above conditions and results, and

$$
\frac{{ }^{\prime}{ }_{i \zeta,-1} i \zeta,-1}{T^{2}} \stackrel{(N, T)_{j}}{=} \frac{1}{(1-\theta)^{2}} \int_{0}^{1} W_{i}^{2}(r) d r .
$$

Thus, under Assumptions 1-4 and rank condition (9), using Order Results in (A.2) and results of Propositions 17.3 and 18.1 of Hamilton (1994) and assuming $\overline{\mathbf{z}}_{0}=\mathbf{0}$ or re-defining $\mathbf{z}_{i t}$ as the deviation from $\overline{\mathbf{z}}_{0}$, as $N$ and $T \rightarrow \infty$ (sequentially and) jointly such that $\sqrt{T} / N \rightarrow 0$, we obtain

$$
t_{i}(N, T) \stackrel{(N, T)_{j}}{\Longrightarrow} \frac{\frac{1}{1-\theta} \int_{0}^{1} W_{i}(r) d W_{i}(r)-\boldsymbol{\omega}_{i f}^{\prime} \boldsymbol{\Lambda}_{f^{*}}^{\prime}\left(\boldsymbol{\Lambda}_{f^{*}} \mathbf{G}_{f} \boldsymbol{\Lambda}_{f^{*}}^{\prime}\right)^{-} \frac{1}{1-\theta} \boldsymbol{\Lambda}_{f^{*}} \boldsymbol{\pi}_{i f}}{\left(\frac{1}{(1-\theta)^{2}} \int_{0}^{1} W_{i}^{2}(r) d r-\frac{1}{1-\theta} \boldsymbol{\pi}_{i f}^{\prime} \boldsymbol{\Lambda}_{\mathbf{f}^{*}}^{\prime}\left(\boldsymbol{\Lambda}_{f^{*}} \mathbf{G}_{f} \boldsymbol{\Lambda}_{f^{*}}^{\prime}\right)^{-} \frac{1}{1-\theta} \boldsymbol{\Lambda}_{f^{*}} \boldsymbol{\pi}_{i f}\right)^{1 / 2}}
$$

which reduces to the case where $\theta=0$. 


\section{References}

Andrews, D. \& C. Monahan (1992) Heteroskedasticity and autocorrelation consistent covariance matrix estimation. Econometrica 60, 953-966.

Bai, J. \& S. Ng (2002) Determining the number of factors in approximate factor models. Econometrica $70,191-221$.

Bai, J. \& S. Ng (2004) A panic on unit root tests and cointegration. Econometrica 72, 1127-1177.

Bai, J., and S. Ng (2007) Panel unit root tests with cross section dependence: A further investigation, mimeo.

Baltagi, B.H \& C. Kao (2000) Nonstationary panels, cointegration in panels and dynamic panels. A Survey, in: B. Baltagi (ed.), Nonstationary panels, panel cointegration, and dynamic panels, Advances in Econometrics 15, 7-52, Amsterdam: JAI Press.

Banerjee, A. (1999) Panel data unit roots and cointegration: An overview. Oxford Bulletin of Economics and Statistics 61, 607-629.

Breitung, J. \& S. Das (2007) Testing for unit roots in panels with a factor structure. Forthcoming in Econometric Theory.

Breitung, J. \& M. H. Pesaran (2007) Unit roots and cointegration in panels. Forthcoming in L. Matyas, and P. Sevestre, The econometrics of panel data (Third Edition), Kluwer Academic Publishers (forthcoming).

Chang, Y. (2002) Nonlinear IV unit root tests in panels with cross sectional dependency. Journal of Econometrics, 110, 261-292.

Choi, I. (2004) Nonstationary panels. Palgrave Handbooks of Econometrics, Vol. 1.

Dees, S., F. di Mauro, M.H. Pesaran \& L.V. Smith (2007) Exploring the international linkages of the euro area: a global VAR analysis. Journal of Applied Econometrics 22, 1-38.

Elliott, G., Rothenberg, T. J. and Stock, J.H. (1996) Efficient tests for an autoregressive unit root. Econometrica, 64, 813-836.

Fuller, W.A. and H.J. Park (1995). Alternative estimators and unit root tests for the autoregressive process. Journal of Time Series Analysis, 16, 415-429.

Gengenbach, C., F.C. Palm \& J-P, Urbain (2006) Panel unit root tests in the presence of cross-sectional dependencies: Comparison and implications for modelling, mimeo.

Hamilton, J. D. (1994) Time Series Analysis. Princeton N.J.: Princeton University Press. 
Im, K.S, M.H. Pesaran \& Y. Shin (2003) Testing for unit roots in heterogeneous panels. Journal of Econometrics 115, 53-74.

Levin, A., C. Lin, and C.J. Chu (2002), Unit root tests in panel data: Asymptotic and finitesample properties. Journal of Econometrics, 108, 1-24.

Leybourne, S.J. (1995) Testing for unit roots using forward and reverse Dickey-Fuller regressions. Oxford Bulletin of Economics and Statistics, 57, 559-571.

Magnus, J.R. \& H. Neudecker (1999) Matrix differential calculus: with applications in statistics and econometrics. Revised edition, John Wiley and Sons.

Moon, H.R. \& B. Perron (2004) Testing for a unit root in panels with dynamic factors. Journal of Econometrics 122, 81-126.

Moon, H.R., B. Perron \& P.C.B. Phillips (2006) Incidental trends and the power of panel unit root tests. Journal of Econometrics doi:10.1016/j.jeconom.2006.10.003.

Pesaran, M. H. (2007) A simple panel unit root test in the presence of cross section dependence. Journal of Applied Econometrics 22, 265-312.

Philllips, P.C.B \& D. Sul (2003) Dynamic panel estimation and homogeneity testing under cross section dependence. Econometrics Journal 6, 217-259.

Smith, L.V., S. Leybourne, T-H. Kim \& P. Newbold (2004) More powerful panel unit root tests with an application to mean reversion in real exchange rates. Journal of Applied Econometrics $19,147-170$.

Westerlund, J. (2007) Panel cointegration tests of the Fisher hypothesis. Forthcoming in Journal of Applied Econometrics.

Westerlund, J. \& R. Larsson (2007) A note on the pooling of individual PANIC unit root tests, mimeo. 
Table 1a: Critical Values of Average of Individual Cross-Sectionally Augmented Dickey-Fuller Distribution (Case I: No Intercept and No Trend)

\begin{tabular}{|c|c|c|c|c|c|c|c|c|c|c|c|c|c|c|c|c|c|c|c|}
\hline \multicolumn{20}{|c|}{$k+1=1$} \\
\hline \multirow[b]{2}{*}{$p$} & \multirow[b]{2}{*}{$(\mathrm{T}, \mathrm{N})$} & \multicolumn{6}{|c|}{$1 \%(\overline{C A D F})$} & \multicolumn{6}{|c|}{$5 \%(\overline{C A D F})$} & \multicolumn{6}{|c|}{$10 \%(\overline{C A D F})$} \\
\hline & & 20 & 30 & 50 & 70 & 100 & 200 & 20 & 30 & 50 & 70 & 100 & 200 & 20 & 30 & 50 & 70 & 100 & 200 \\
\hline & 20 & -1.81 & -1.73 & -1.67 & -1.65 & -1.61 & -1.58 & -1.60 & -1.55 & -1.51 & -1.51 & -1.49 & -1.48 & -1.48 & -1.46 & -1.43 & -1.42 & -1.42 & -1.41 \\
\hline & 30 & -1.79 & -1.72 & -1.67 & -1.63 & -1.61 & -1.60 & -1.60 & -1.56 & -1.53 & -1.51 & -1.50 & -1.50 & -1.49 & -1.46 & -1.44 & -1.43 & -1.43 & -1.43 \\
\hline 0 & 50 & -1.79 & -1.72 & -1.68 & -1.64 & -1.63 & -1.60 & -1.61 & -1.56 & -1.54 & -1.52 & -1.51 & -1.50 & -1.50 & -1.47 & -1.45 & -1.44 & -1.44 & -1.42 \\
\hline & 70 & -1.81 & -1.73 & -1.67 & -1.65 & -1.63 & -1.60 & -1.61 & -1.56 & -1.53 & -1.53 & -1.51 & -1.50 & -1.50 & -1.46 & -1.44 & -1.45 & -1.43 & -1.43 \\
\hline & 100 & -1.78 & -1.72 & -1.66 & -1.65 & -1.64 & -1.60 & -1.60 & -1.56 & -1.54 & -1.52 & -1.52 & -1.50 & -1.48 & -1.46 & -1.44 & -1.44 & -1.44 & -1.43 \\
\hline & 200 & -1.80 & -1.73 & -1.68 & -1.64 & -1.63 & -1.60 & -1.60 & -1.57 & -1.54 & -1.53 & -1.51 & -1.50 & -1.49 & -1.47 & -1.46 & -1.45 & -1.44 & -1.43 \\
\hline & 20 & -1.82 & -1.74 & -1.69 & -1.66 & -1.62 & -1.59 & -1.61 & -1.56 & -1.51 & -1.50 & -1.49 & -1.47 & -1.48 & -1.45 & -1.42 & -1.42 & -1.41 & -1.40 \\
\hline & 30 & -1.80 & -1.74 & -1.67 & -1.65 & -1.62 & -1.61 & -1.60 & -1.57 & -1.53 & -1.51 & -1.50 & -1.50 & -1.49 & -1.46 & -1.44 & -1.42 & -1.43 & -1.42 \\
\hline 1 & 50 & -1.80 & -1.73 & -1.68 & -1.65 & -1.63 & -1.60 & -1.61 & -1.57 & -1.54 & -1.52 & -1.52 & -1.50 & -1.50 & -1.47 & -1.45 & -1.44 & -1.44 & -1.42 \\
\hline & 70 & -1.80 & -1.72 & -1.68 & -1.66 & -1.64 & -1.61 & -1.61 & -1.56 & -1.54 & -1.53 & -1.51 & -1.50 & -1.50 & -1.46 & -1.44 & -1.45 & -1.43 & -1.43 \\
\hline & 100 & -1.79 & -1.72 & -1.67 & -1.65 & -1.64 & -1.61 & -1.60 & -1.57 & -1.54 & -1.52 & -1.52 & -1.51 & -1.49 & -1.47 & -1.45 & -1.44 & -1.44 & -1.43 \\
\hline & 200 & -1.79 & -1.74 & -1.68 & -1.65 & -1.63 & -1.61 & -1.60 & -1.57 & -1.54 & -1.53 & -1.51 & -1.51 & -1.49 & -1.47 & -1.46 & -1.45 & -1.44 & -1.43 \\
\hline & 20 & -1.74 & -1.67 & -1.62 & -1.59 & -1.56 & -1.53 & -1.52 & -1.47 & -1.43 & -1.42 & -1.40 & -1.38 & -1.39 & -1.36 & -1.33 & -1.32 & -1.31 & -1.31 \\
\hline & 30 & -1.77 & -1.70 & -1.63 & -1.59 & -1.58 & -1.57 & 1.56 & -1.51 & -1.48 & -1.46 & -1.45 & -1.45 & -1.44 & -1.40 & -1.38 & -1.36 & -1.37 & -1.36 \\
\hline 2 & 50 & -1.77 & -1.70 & -1.65 & -1.62 & -1.61 & -1.58 & -1.58 & -1.53 & -1.51 & -1.49 & -1.48 & -1.47 & -1.47 & -1.43 & -1.42 & -1.40 & -1.40 & -1.39 \\
\hline & 70 & -1.78 & -1.70 & -1.66 & -1.63 & -1.62 & -1.59 & -1.58 & -1.54 & -1.51 & -1.51 & -1.49 & -1.48 & -1.47 & -1.44 & -1.42 & -1.42 & -1.41 & -1.40 \\
\hline & 100 & -1.78 & -1.72 & -1.65 & -1.65 & -1.62 & -1.59 & -1.59 & -1.54 & -1.52 & -1.51 & -1.50 & -1.49 & -1.47 & -1.45 & -1.43 & -1.43 & -1.42 & -1.42 \\
\hline & 200 & -1.78 & -1.72 & -1.67 & -1.64 & -1.62 & -1.60 & -1.59 & -1.56 & -1.53 & -1.52 & -1.50 & -1.50 & -1.49 & -1.46 & -1.45 & -1.44 & -1.43 & -1.43 \\
\hline & 20 & -1.75 & -1.70 & -1.63 & -1.60 & -1.57 & -1.54 & -1.51 & -1.48 & -1.43 & -1.42 & -1.40 & -1.38 & -1.39 & -1.35 & -1.32 & -1.31 & -1.31 & -1.30 \\
\hline & 30 & -1.77 & -1.71 & -1.66 & -1.63 & -1.61 & -1.59 & -1.56 & -1.52 & -1.48 & -1.46 & -1.46 & -1.45 & -1.44 & -1.40 & -1.38 & -1.36 & -1.36 & -1.36 \\
\hline 3 & 50 & -1.78 & -1.71 & -1.65 & -1.63 & -1.63 & -1.60 & -1.58 & -1.54 & -1.52 & -1.49 & -1.49 & -1.48 & -1.47 & -1.44 & -1.42 & -1.40 & -1.40 & -1.39 \\
\hline & 70 & -1.78 & -1.72 & -1.67 & -1.64 & -1.63 & -1.60 & 1.58 & -1.54 & -1.52 & -1.51 & -1.49 & -1.48 & -1.47 & -1.43 & -1.42 & -1.42 & -1.41 & -1.41 \\
\hline & 100 & -1.79 & -1.72 & -1.66 & -1.65 & -1.64 & -1.60 & -1.59 & -1.55 & -1.53 & -1.51 & -1.51 & -1.50 & -1.47 & -1.45 & -1.43 & -1.43 & -1.43 & -1.42 \\
\hline & 200 & -1.78 & -1.73 & -1.67 & -1.64 & -1.62 & -1.61 & -1.60 & -1.56 & -1.54 & -1.52 & -1.51 & -1.50 & -1.49 & -1.47 & -1.45 & -1.44 & -1.43 & -1.43 \\
\hline & 20 & -1.68 & -1.60 & -1.55 & -1.53 & -1.49 & -1.46 & -1.44 & -1.40 & -1.35 & -1.34 & -1.33 & -1.30 & -1.29 & -1.27 & -1.24 & -1.23 & -1.22 & -1.20 \\
\hline & 30 & -1.76 & -1.65 & -1.62 & -1.58 & -1.56 & -1.54 & -1.51 & -1.46 & -1.43 & -1.41 & -1.40 & -1.40 & -1.39 & -1.35 & -1.33 & -1.30 & -1.31 & -1.31 \\
\hline 4 & 50 & -1.75 & -1.69 & -1.64 & -1.61 & -1.60 & -1.59 & -1.55 & -1.50 & -1.49 & -1.46 & -1.46 & -1.45 & -1.44 & -1.40 & -1.39 & -1.37 & -1.37 & -1.36 \\
\hline & 70 & -1.76 & -1.70 & -1.65 & -1.63 & -1.61 & -1.59 & -1.56 & -1.52 & -1.49 & -1.49 & -1.47 & -1.46 & -1.44 & -1.42 & -1.40 & -1.40 & -1.39 & -1.38 \\
\hline & 100 & -1.78 & -1.70 & -1.65 & -1.63 & -1.63 & -1.59 & -1.58 & -1.53 & -1.51 & -1.50 & -1.49 & -1.48 & -1.46 & -1.43 & -1.42 & -1.41 & -1.41 & -1.40 \\
\hline & 200 & -1.77 & -1.72 & -1.66 & -1.64 & -1.62 & -1.60 & 1.59 & -1.55 & -1.53 & -1.51 & -1.50 & -1.49 & 1.48 & -1.46 & -1.44 & -1.43 & -1.42 & -1.42 \\
\hline & & & & & & & & & $k+$ & $1=2$ & & & & & & & & & \\
\hline & & & & $1 \%(\bar{C}$ & $\overline{A D D F})$ & & & & & $5 \%(\bar{C}$ & $\overline{A D F})$ & & & & & $10 \%(\bar{c}$ & $\overline{A D F}$ & & \\
\hline$p$ & $(\mathrm{~T}, \mathrm{~N})$ & 20 & 30 & 50 & 70 & 100 & 200 & 20 & 30 & 50 & 70 & 100 & 200 & 20 & 30 & 50 & 70 & 100 & 200 \\
\hline & 20 & -2.18 & -2.09 & -2.03 & -2.00 & -1.96 & -1.93 & -1.94 & -1.90 & -1.85 & -1.83 & -1.82 & -1.80 & -1.82 & -1.79 & -1.75 & -1.74 & -1.73 & -1.72 \\
\hline & 30 & -2.15 & -2.08 & -2.03 & -1.99 & -1.98 & -1.96 & -1.95 & -1.90 & -1.87 & -1.85 & -1.83 & -1.82 & -1.84 & -1.79 & -1.77 & -1.76 & -1.75 & -1.74 \\
\hline 0 & 50 & -2.15 & -2.07 & -2.03 & -2.01 & -1.99 & -1.97 & -1.96 & -1.91 & -1.87 & -1.86 & -1.84 & -1.83 & -1.85 & -1.81 & -1.79 & -1.77 & -1.76 & -1.75 \\
\hline & 70 & .16 & -2.09 & -2.05 & -2.01 & -2.00 & -1.97 & -1.95 & -1.92 & -1.88 & -1.86 & -1.86 & -1.83 & -1.85 & -1.82 & -1.79 & -1.78 & -1.77 & -1.76 \\
\hline & 100 & -2.15 & -2.08 & -2.05 & -2.01 & -2.01 & -1.98 & 1.96 & -1.92 & -1.89 & -1.87 & -1.87 & -1.85 & -1.86 & -1.83 & -1.80 & -1.79 & -1.78 & -1.77 \\
\hline & 200 & -2.16 & -2.09 & -2.04 & -2.04 & -2.00 & -1.98 & -1.97 & -1.93 & -1.89 & -1.88 & -1.87 & -1.85 & -1.86 & -1.83 & -1.80 & -1.79 & -1.78 & -1.77 \\
\hline & 20 & -2.15 & -2.09 & -2.01 & -1.97 & -1.94 & -1.90 & -1.90 & -1.85 & -1.81 & -1.79 & -1.77 & -1.75 & -1.76 & -1.74 & -1.69 & -1.68 & -1.68 & -1.66 \\
\hline & 30 & -2.15 & -2.07 & -2.02 & -1.98 & -1.96 & -1.95 & -1.93 & -1.87 & -1.83 & -1.81 & -1.80 & -1.80 & -1.81 & -1.76 & -1.74 & -1.73 & -1.71 & -1.71 \\
\hline 1 & 50 & -2.14 & -2.07 & -2.03 & -2.00 & -1.99 & -1.96 & -1.95 & -1.90 & -1.86 & -1.85 & -1.83 & -1.82 & -1.84 & -1.80 & -1.77 & -1.76 & -1.75 & -1.74 \\
\hline & 70 & -2.14 & -2.10 & -2.04 & -2.00 & -1.99 & -1.96 & -1.95 & -1.91 & -1.87 & -1.86 & -1.85 & -1.83 & -1.84 & -1.80 & -1.78 & -1.77 & -1.76 & -1.75 \\
\hline & 100 & -2.15 & -2.09 & -2.05 & -2.02 & -2.00 & -1.97 & -1.96 & -1.92 & -1.88 & -1.86 & -1.86 & -1.84 & -1.85 & -1.82 & -1.79 & -1.78 & -1.77 & -1.76 \\
\hline & 200 & -2.16 & -2.09 & -2.04 & -2.04 & -2.00 & -1.98 & -1.97 & -1.93 & -1.89 & -1.88 & -1.87 & -1.85 & -1.85 & -1.82 & -1.80 & -1.79 & -1.78 & -1.77 \\
\hline & 20 & -2.01 & -1.94 & -1.87 & -1.83 & -1.79 & -1.76 & -1.76 & -1.71 & -1.65 & -1.64 & -1.61 & -1.59 & -1.62 & -1.58 & -1.53 & -1.52 & -1.51 & -1.49 \\
\hline & 30 & -2.06 & -1.97 & -1.92 & -1.89 & -1.87 & -1.86 & -1.83 & -1.77 & -1.75 & -1.72 & -1.70 & -1.70 & -1.70 & -1.66 & -1.64 & -1.62 & -1.61 & -1.60 \\
\hline 2 & 50 & -2.12 & -2.03 & -1.99 & -1.95 & -1.94 & -1.90 & -1.90 & -1.85 & -1.80 & -1.79 & -1.78 & -1.77 & -1.78 & -1.74 & -1.71 & -1.70 & -1.69 & -1.68 \\
\hline & 70 & -2.11 & -2.07 & -2.00 & -1.97 & -1.95 & -1.93 & -1.91 & -1.87 & -1.83 & -1.82 & -1.81 & -1.79 & -1.80 & -1.76 & -1.74 & -1.73 & -1.72 & -1.71 \\
\hline & 100 & -2.13 & -2.06 & -2.04 & -1.99 & -1.98 & -1.94 & -1.94 & -1.89 & -1.86 & -1.84 & -1.84 & -1.81 & -1.81 & -1.79 & -1.76 & -1.75 & -1.74 & -1.73 \\
\hline & 200 & -2.14 & -2.08 & -2.03 & -2.03 & -1.99 & -1.97 & -1.96 & -1.91 & -1.88 & -1.86 & -1.85 & -1.83 & -1.84 & -1.81 & -1.79 & -1.77 & -1.77 & -1.76 \\
\hline & 20 & -2.02 & -1.91 & -1.81 & -1.78 & -1.74 & -1.72 & -1.72 & -1.67 & -1.59 & -1.56 & -1.54 & -1.52 & -1.57 & -1.53 & -1.46 & -1.45 & -1.43 & -1.41 \\
\hline & 30 & -2.04 & -1.95 & -1.91 & -1.89 & -1.85 & -1.83 & -1.79 & -1.74 & -1.71 & -1.69 & -1.66 & -1.67 & -1.66 & -1.61 & -1.60 & -1.58 & -1.57 & -1.57 \\
\hline 3 & 50 & -2.10 & -2.03 & -1.97 & -1.94 & -1.92 & -1.89 & -1.88 & -1.82 & -1.79 & -1.78 & -1.76 & -1.76 & -1.76 & -1.72 & -1.70 & -1.68 & -1.67 & -1.67 \\
\hline & 70 & -2.11 & -2.07 & -2.00 & -1.96 & -1.95 & -1.93 & -1.90 & -1.87 & -1.83 & -1.81 & -1.80 & -1.78 & -1.79 & -1.76 & -1.73 & -1.71 & -1.71 & -1.70 \\
\hline & 100 & -2.13 & -2.07 & -2.03 & -2.00 & -1.98 & -1.94 & -1.93 & -1.89 & -1.85 & -1.84 & -1.83 & -1.81 & -1.81 & -1.78 & -1.75 & -1.74 & -1.74 & -1.72 \\
\hline & 200 & -2.14 & -2.08 & -2.03 & -2.03 & -1.99 & -1.97 & -1.95 & -1.91 & -1.88 & -1.86 & -1.85 & -1.84 & -1.84 & -1.81 & -1.78 & -1.77 & -1.76 & -1.76 \\
\hline & 20 & -2.29 & -2.10 & -1.92 & -1.81 & -1.78 & -1.67 & -1.78 & -1.65 & -1.57 & -1.53 & -1.50 & -1.44 & -1.55 & -1.47 & -1.42 & -1.39 & -1.35 & -1.32 \\
\hline & 30 & -1.94 & -1.85 & -1.81 & -1.77 & -1.74 & -1.75 & -1.70 & -1.63 & -1.61 & -1.58 & -1.56 & -1.56 & -1.56 & -1.51 & -1.50 & -1.47 & -1.46 & -1.45 \\
\hline 4 & 50 & -2.05 & -1.97 & -1.92 & -1.89 & -1.88 & -1.85 & -1.83 & -1.78 & -1.74 & -1.72 & -1.71 & -1.70 & -1.70 & -1.66 & -1.64 & -1.62 & -1.61 & -1.61 \\
\hline & 70 & -2.07 & -2.03 & -1.97 & -1.94 & -1.92 & -1.90 & -1.87 & -1.83 & -1.79 & -1.78 & -1.77 & -1.74 & -1.74 & -1.72 & -1.69 & -1.68 & -1.67 & -1.66 \\
\hline & 100 & -2.10 & -2.04 & -2.01 & -1.97 & -1.96 & -1.92 & -1.90 & -1.86 & -1.83 & -1.81 & -1.81 & -1.79 & -1.78 & -1.75 & -1.72 & -1.72 & -1.71 & -1.70 \\
\hline & 200 & -2.13 & -2.07 & -2.02 & -2.01 & -1.98 & -1.96 & -1.94 & -1.90 & -1.86 & -1.85 & -1.84 & -1.82 & -1.82 & -1.80 & -1.77 & -1.76 & -1.75 & -1.74 \\
\hline
\end{tabular}




\begin{tabular}{|c|c|c|c|c|c|c|c|c|c|c|c|c|c|c|c|c|c|c|c|}
\hline & & & & & & & & & & & & & & & & & & онитие & \\
\hline & & & & & & & & & $k+$ & $-1=3$ & & & & & & & & & \\
\hline & & & & $1 \%(\bar{C}$ & $A D F$ & & & & & $5 \%(\bar{C}$ & $A D F$ & & & & & $10 \%(\bar{C}$ & $\overline{C A D F})$ & & \\
\hline$p$ & $(\mathrm{~T}, \mathrm{~N})$ & 20 & 30 & 50 & 70 & 100 & 200 & 20 & 30 & 50 & 70 & 100 & 200 & 20 & 30 & 50 & 70 & 100 & 200 \\
\hline & 20 & -2.40 & -2.34 & -2.26 & -2.23 & -2.19 & -2.17 & \begin{tabular}{|l|}
-2.19 \\
\end{tabular} & -2.14 & -2.09 & -2.06 & -2.04 & -2.02 & -2.06 & -2.02 & -1.98 & -1.97 & -1.96 & -1.94 \\
\hline & 30 & -2.42 & -2.37 & -2.29 & -2.25 & -2.23 & -2.20 & -2.21 & -2.15 & -2.12 & -2.10 & -2.09 & -2.06 & -2.09 & -2.05 & -2.03 & -2.01 & -2.00 & -1.98 \\
\hline 0 & 50 & -2.44 & -2.34 & -2.30 & -2.26 & -2.24 & -2.23 & -2.24 & -2.18 & -2.14 & -2.13 & -2.11 & -2.10 & -2.13 & -2.09 & -2.05 & -2.04 & -2.03 & -2.02 \\
\hline & 70 & -2.44 & -2.36 & -2.33 & -2.27 & -2.27 & -2.23 & -2.25 & -2.19 & -2.16 & -2.14 & -2.13 & -2.10 & -2.13 & -2.10 & -2.07 & -2.05 & -2.05 & -2.03 \\
\hline & 100 & -2.45 & -2.36 & -2.31 & -2.28 & -2.27 & -2.25 & -2.25 & -2.20 & -2.16 & -2.15 & -2.13 & -2.12 & -2.14 & -2.10 & -2.07 & -2.07 & -2.05 & -2.04 \\
\hline & 200 & -2.44 & -2.38 & -2.32 & -2.30 & -2.27 & -2.26 & -2.25 & -2.21 & -2.18 & -2.16 & -2.14 & -2.13 & -2.14 & -2.11 & -2.09 & -2.07 & -2.06 & -2.05 \\
\hline & 20 & -2.35 & -2.23 & -2.16 & -2.14 & -2.12 & -2.07 & \begin{tabular}{|l|}
-2.08 \\
\end{tabular} & -2.02 & -1.96 & -1.94 & -1.92 & -1.90 & -1.94 & -1.90 & -1.85 & -1.84 & -1.82 & -1.80 \\
\hline & 30 & -2.38 & -2.28 & -2.24 & -2.20 & -2.17 & -2.14 & -2.14 & -2.09 & -2.05 & -2.02 & -2.02 & -1.99 & -2.01 & -1.98 & -1.95 & -1.93 & -1.92 & -1.90 \\
\hline 1 & 50 & -2.41 & -2.32 & -2.28 & -2.23 & -2.23 & -2.19 & -2.20 & -2.15 & -2.11 & -2.09 & -2.07 & -2.06 & -2.08 & -2.05 & -2.01 & -2.00 & -1.98 & -1.97 \\
\hline & 70 & -2.42 & -2.34 & -2.31 & -2.25 & -2.26 & -2.21 & -2.21 & -2.17 & -2.13 & -2.11 & -2.10 & -2.08 & -2.10 & -2.07 & -2.04 & -2.02 & -2.02 & -2.00 \\
\hline & 100 & -2.44 & -2.36 & -2.30 & -2.28 & -2.25 & -2.24 & -2.24 & -2.19 & -2.14 & -2.13 & -2.11 & -2.10 & -2.12 & -2.09 & -2.05 & -2.05 & -2.03 & -2.02 \\
\hline & 200 & -2.42 & -2.38 & -2.32 & -2.30 & -2.27 & -2.25 & -2.24 & -2.21 & -2.17 & -2.15 & -2.13 & -2.13 & -2.14 & -2.11 & -2.09 & -2.07 & -2.05 & -2.04 \\
\hline & 20 & -2.20 & -2.09 & -1.98 & -1.92 & -1.86 & -1.83 & \begin{tabular}{|l|} 
\\
\end{tabular} & -1.81 & -1.73 & -1.70 & -1.68 & -1.64 & -1.72 & -1.67 & -1.61 & -1.58 & -1.56 & -1.54 \\
\hline & 30 & -2.25 & $\begin{array}{l}-2.17 \\
\end{array}$ & -2.08 & -2.03 & -2.03 & -1.98 & $\begin{array}{l}-1.99 \\
\end{array}$ & -1.94 & -1.89 & -1.86 & -1.85 & -1.82 & -1 & -1.81 & -1.78 & -1.76 & -1.75 & -1.73 \\
\hline 2 & 50 & -2.33 & $\begin{array}{l}-2.24 \\
\end{array}$ & $\begin{array}{l}-2.20 \\
\end{array}$ & -2.16 & -2.14 & -2.11 & $\begin{array}{l}-2.11 \\
\end{array}$ & -2.06 & $\begin{array}{l}-2.02 \\
\end{array}$ & $\begin{array}{l}-2.00 \\
\end{array}$ & -1.98 & -1.97 & -1 . & & -1.92 & -1.91 & & -1.88 \\
\hline & 70 & .35 & -2.28 & -2.25 & -2.21 & -2.20 & -2.16 & -2.15 & -2.12 & -2.07 & -2.05 & -2.04 & -2.02 & -2.04 & -2.01 & -1.98 & -1.96 & -1.95 & -1.94 \\
\hline & 100 & -2.40 & -2.31 & -2.26 & -2.24 & -2.22 & -2.20 & -2.20 & -2.15 & -2.10 & -2.09 & -2.07 & -2.05 & -2 & -2 & -2.01 & -2.00 & -1.99 & -1.98 \\
\hline & 200 & -2.42 & -2.36 & -2.30 & -2.28 & -2.24 & -2.23 & -2.22 & -2.19 & -2.15 & -2.13 & -2.11 & -2.11 & -2.11 & -2.09 & -2.06 & -2.04 & -2.03 & -2.02 \\
\hline & 20 & - & & - & 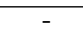 & - & & & & & - & & & & & - & & & \\
\hline & 30 & -2.17 & -2.05 & -1.99 & -1.94 & -1.93 & -1.90 & -1.90 & -1.82 & -1.78 & -1.75 & -1.74 & -1.72 & -1.76 & -1.70 & -1.67 & -1.65 & -1.64 & -1.62 \\
\hline 3 & 50 & -2 & -2.23 & -2.15 & -2.12 & -2 . & -2.0 & -2 . & -2.02 & -1.97 & -1.95 & -1 & -1.92 & -1 . & & -1.86 & -1.86 & 84 & -1.83 \\
\hline & 70 & -2.33 & -2.26 & -2.24 & -2.19 & -2.19 & -2.14 & -2.12 & -2.09 & -2.05 & -2.02 & -2.01 & -1.99 & -2.01 & -1 & -1.95 & -1.93 & -1.92 & -1.91 \\
\hline & 100 & -2.39 & -2.29 & -2.25 & -2.23 & -2.21 & -2.18 & -2.18 & -2.13 & -2.09 & -2.07 & -2 . & -2.04 & -2 & -2 & -2.00 & -1.98 & -1.97 & -1.96 \\
\hline & 200 & -2.40 & -2.35 & -2.30 & -2.28 & -2.24 & -2.23 & -2.21 & -2.18 & -2.14 & -2.12 & -2.10 & -2.10 & -2.11 & -2.08 & -2.05 & -2.04 & -2.03 & -2.01 \\
\hline & 20 & & & & & & & & & & & & & & & & & & \\
\hline & 30 & -2.00 & -1.89 & -1.82 & -1.77 & -1.75 & -1.69 & .73 & -1.65 & -1.61 & -1. & -1 & -1.52 & -1 . & & -1.49 & -1.46 & -1.44 & -1.42 \\
\hline 4 & 50 & .23 & -2.15 & -2.06 & -2.04 & -2.02 & -2.00 & -1.98 & -1.94 & -1.87 & -1.86 & -1.84 & -1.83 & -1 . & -1 & -1.78 & -1.76 & -1.74 & -1.73 \\
\hline & 70 & -2.27 & -2.21 & -2.18 & -2.14 & -2.13 & -2.08 & -2.07 & -2.02 & -1.99 & -1.96 & -1.95 & -1.93 & -1.95 & -1.91 & -1.89 & -1.87 & -1.86 & -1.84 \\
\hline & 100 & -2.35 & -2.26 & -2.22 & -2.19 & -2.17 & -2.15 & -2.14 & -2.08 & -2.05 & -2.03 & -2.01 & -2.00 & -2 & -1 & -1.95 & -1.94 & -1.93 & -1.91 \\
\hline & 200 & -2.39 & -2.33 & -2.27 & -2.27 & -2.22 & -2.21 & -2.19 & -2.16 & -2.12 & -2.11 & -2.08 & -2.08 & -2.08 & -2.06 & -2.04 & -2.01 & -2.01 & -1.99 \\
\hline & & & & & & & & & $k t$ & $-1=4$ & & & & & & & & & \\
\hline & & & & $1 \%(\bar{C}$ & $A D F$ & & & & & $5 \%(\bar{C}$ & $A D F$ & & & & & $10 \%$ & $A D F$ & & \\
\hline$p$ & $(\mathrm{~T}, \mathrm{~N})$ & 20 & 30 & 50 & 70 & 100 & 200 & 20 & 30 & 50 & 70 & 100 & 200 & 20 & 30 & 50 & 70 & 100 & 200 \\
\hline & 20 & -2.59 & -2.52 & -2.45 & -2.41 & -2.38 & -2.34 & -2.37 & -2.32 & -2.26 & -2.23 & -2.22 & -2.19 & -2.24 & -2.20 & -2.15 & -2.14 & -2.12 & -2.10 \\
\hline & 30 & -2.64 & -2.53 & -2.48 & -2.44 & -2.43 & -2.39 & -2.41 & -2.36 & -2.32 & -2.28 & -2.28 & -2.25 & -2.30 & -2.25 & -2.22 & -2.20 & -2.19 & -2.17 \\
\hline 0 & 50 & -2.65 & -2.57 & -2.53 & -2.48 & -2.47 & -2.44 & -2.45 & -2.40 & -2.37 & -2.34 & -2.33 & -2.31 & -2.34 & -2.31 & -2.28 & -2.26 & -2.25 & -2.24 \\
\hline & 70 & -2 & -2.59 & -2.55 & -2.50 & -2.49 & -2.46 & -2.48 & -2.42 & -2.39 & -2.36 & -2 & -2.33 & -2 & -2 & -2.30 & -2.28 & -2.27 & -2.25 \\
\hline & 100 & -2.68 & -2.60 & -2.54 & -2.51 & -2.49 & -2.46 & -2.48 & -2.44 & -2.39 & -2.37 & -2.36 & -2.34 & -2.38 & -2.35 & -2.31 & -2.30 & -2.29 & -2.27 \\
\hline & 200 & -2.68 & -2.61 & -2.56 & -2.53 & -2.52 & -2. & -2 & -2.44 & -2.4 & -2.40 & -2 & -2.36 & -2 & -2 & -2 & -2.31 & -2.31 & -2.29 \\
\hline & 20 & -2.52 & -2.38 & -2.30 & -2.25 & -2.19 & -2.18 & -2.18 & -2.11 & -2.05 & -2.02 & -2.00 & -1.96 & -2.03 & -1.98 & -1.92 & -1.91 & -1.88 & -1.86 \\
\hline & 30 & -2.54 & -2.44 & -2.37 & -2.34 & -2.31 & -2.27 & -2 & -2.23 & -2.1 & -2.16 & -2 & -2.11 & & & & -2.06 & -2.04 & -2.03 \\
\hline 1 & 50 & -2.59 & -2.52 & -2.46 & -2.42 & -2.40 & -2.38 & -2.39 & -2.33 & -2.30 & -2.27 & -2.25 & -2.24 & -2.28 & -2.23 & -2.20 & -2.19 & -2.17 & -2.16 \\
\hline & 70 & -2.6 & -2.54 & -2.50 & -2.46 & -2.45 & -2.4 & -2 & -2.37 & -2.3 & -2 & -2 & -2. & -2 & & & & & -2.20 \\
\hline & 100 & -2.64 & -2.58 & -2.50 & -2.49 & -2.46 & -2.42 & -2.45 & -2.41 & -2.36 & -2.34 & -2.33 & -2.31 & -2.35 & -2.31 & -2.27 & -2.26 & -2.25 & -2.24 \\
\hline & 200 & -2.67 & -2.61 & -2.54 & -2.52 & -2.51 & -2.47 & -2.48 & -2.43 & -2.39 & -2.38 & -2.37 & -2.35 & -2.38 & -2.34 & -2.31 & -2.30 & -2.29 & -2.28 \\
\hline & 20 & & & & & - & & & & & & & & & & & & & \\
\hline & 30 & & -2.23 & -2.13 & -2.0 & & -2.0 & & & & & & & & & & & & -1.77 \\
\hline 2 & 50 & -2.48 & -2.41 & -2.35 & -2.30 & -2.2 & -2.2 & -2 & -2.21 & -2.17 & -2.14 & -2.13 & -2 . & -2 & -2 & -2.07 & -2.06 & -2.04 & -2.03 \\
\hline & 7( & & -2.4 & -2.43 & -2.38 & -2 . & -2 & -2 . & -2.29 & -2 & -2 . & -2 & & & & & & -2.13 & -2.11 \\
\hline & 100 & -2.6 & -2.53 & -2.45 & -2.44 & -2 . & -2. & -2 & -2.35 & -2 & -2 & -2 . & -2 . & -2 & & -2 & -2 & -2.19 & -2.18 \\
\hline & 200 & -2.65 & -2.57 & -2.52 & -2.50 & -2.49 & -2.44 & -2.45 & -2.40 & -2.36 & -2.36 & -2.34 & -2.32 & -2.35 & -2.31 & -2.28 & -2.27 & -2.26 & -2.25 \\
\hline & 20 & - & & & & & - & & & & & - & & & & & & & \\
\hline & 30 & -2.19 & -2.06 & -1.95 & -1.91 & -1.8 & -1.8 & -1 & -1.80 & -1 & -1. & -1.6 & & & & & & & \\
\hline 3 & 50 & -2.42 & -2.34 & -2.25 & -2.22 & -2.2 & -2.1 & -2 . & -2.12 & -2. & -2. & -2 . & -2.02 & -2 . & & -1 . & -1 . & -1.94 & -1.93 \\
\hline & 70 & -2.5 & -2.42 & -2.38 & -2.34 & -2.3 & -2.2 & -2 . & -2.24 & -2 & -2 & -2 & -2 . & -2 & & & & & -2.06 \\
\hline & 100 & -2.5 & $\begin{array}{l}-2.49 \\
\end{array}$ & -2.43 & $\begin{array}{l}-2.40 \\
\end{array}$ & -2.3 & -2.3 & -2 . & -2.31 & -2 & -2. & -2 & $\begin{array}{l}-2.22 \\
\end{array}$ & -2 & & -2.18 & -2.16 & -2.15 & -2.14 \\
\hline & 200 & -2.63 & -2.57 & -2.50 & -2.49 & -2.49 & -2.43 & -2.43 & -2.39 & -2.34 & -2.33 & -2.32 & -2.30 & -2.33 & -2.29 & -2.26 & -2.25 & -2.24 & -2.23 \\
\hline & 20 & & & & & & & & & & & & & & & & & & \\
\hline & 30 & & - & - & & - & - & & - & - & - & - & - & - & & - & - & - & \\
\hline 4 & 50 & -2.28 & -2.20 & -2.15 & -2.09 & -2.07 & -2.04 & -2.05 & -1.97 & -1.93 & -1.90 & -1.89 & -1.87 & -1.91 & -1.86 & -1.82 & -1.81 & -1.80 & -1.78 \\
\hline & 70 & -2.44 & -2.34 & -2.30 & -2.25 & -2.24 & -2.2 & -2 & -2.14 & -2.11 & -2.1 & -2.07 & -2. & -2 & -2.03 & -2.01 & -1 . & -1.98 & -1.9 \\
\hline & 100 & -2.52 & -2.44 & -2.37 & -2.35 & -2.33 & -2.30 & -2.31 & -2.26 & -2.21 & -2.18 & -2.18 & -2.16 & -2.20 & -2.15 & -2.12 & -2.10 & -2.10 & -2.08 \\
\hline & 200 & -2.62 & -2.54 & -2.48 & -2.46 & -2.45 & -2.41 & -2.41 & -2.36 & -2.31 & -2.31 & -2.29 & -2.28 & -2.30 & -2.26 & -2.23 & -2.22 & -2.22 & -2.20 \\
\hline
\end{tabular}

Notes: The critical values are obtained by stochastic simulation. The data generating process is $y_{i t}=y_{i t-1}+f_{t}+u_{i t}$ with $f_{t} \sim \operatorname{iidN}(0,1), u_{i t} \sim \operatorname{iidN}(0,1)$, with $y_{i 0}=0$, and the $j^{\text {th }}$ element of the $k \times 1$ vector of additional regressors, $x_{i t}$, are generated as $x_{i j t}=x_{i j t-1}+f_{j t}+v_{i j t}$, with $f_{j t} \sim i i d N(0,1), v_{i j t} \sim i i d N(0,1)$ and $x_{i j 0}=0, i=1,2, \ldots, N ; j=1,2, \ldots, k$; $t=-p, \ldots, T$. Based on these data, $C A D F_{i}$, which is the $t$-ratio of coefficient on $y_{i t-1}$ of regression of $\Delta y_{i t}$ on $y_{i t-1}$, 
$w_{i t, p}^{\prime}=\left(\bar{z}_{t-1}^{\prime} ; \Delta \bar{z}_{t}^{\prime}, \Delta \bar{z}_{t-1}^{\prime}, \ldots, \Delta \bar{z}_{t-p}^{\prime} ; \Delta y_{i, t-1}, \ldots, \Delta y_{i, t-p}\right)$ with $\bar{z}_{t}=N^{-1} \sum_{i=1}^{N}\left(y_{i t}, x_{i t}^{\prime}\right)^{\prime}$ and deterministics as specified, then $\overline{C A D F}=N^{-1} \sum_{i=1}^{N} C A D F_{i}$ are computed. $(100 \times \alpha) \%$ critical values are obtained as the $1-\alpha$ quantiles of $\overline{C A D F}$ for $\alpha=0.01,0.05,0.1$. Computations are based on 10000 replications. 
Table 1b: Critical Values of Average of Individual Cross-Sectionally Augmented Dickey-Fuller Distribution (Case II: Intercept Only)

\begin{tabular}{|c|c|c|c|c|c|c|c|c|c|c|c|c|c|c|c|c|c|c|c|}
\hline \multicolumn{20}{|c|}{$k+1=1$} \\
\hline \multirow[b]{2}{*}{$p$} & \multirow[b]{2}{*}{$(\mathrm{T}, \mathrm{N})$} & \multicolumn{6}{|c|}{$1 \%(\overline{C A D F})$} & \multicolumn{6}{|c|}{$5 \%(\overline{C A D F})$} & \multicolumn{6}{|c|}{$10 \%(\overline{C A D F})$} \\
\hline & & 20 & 30 & 50 & 70 & 100 & 200 & 20 & 30 & 50 & 70 & 100 & 200 & 20 & 30 & 50 & 70 & 100 & 200 \\
\hline & 20 & -2.39 & -2.32 & -2.23 & -2.20 & -2.18 & -2.14 & -2.20 & -2.15 & -2.10 & -2.08 & -2.06 & -2.04 & -2.10 & -2.06 & -2.03 & -2.01 & -2.00 & -1.99 \\
\hline & 30 & -2.37 & -2.30 & -2.23 & -2.20 & -2.16 & -2.14 & -2.20 & -2.15 & -2.11 & -2.09 & -2.07 & -2.05 & -2.10 & -2.07 & -2.03 & -2.03 & -2.00 & -2.00 \\
\hline 0 & 50 & -2.36 & -2.29 & -2.22 & -2.20 & -2.16 & -2.15 & -2.20 & -2.14 & -2.11 & -2.09 & -2.07 & -2.06 & -2.11 & -2.07 & -2.04 & -2.03 & -2.02 & -2.01 \\
\hline & 70 & -2.36 & -2.29 & -2.22 & -2.20 & -2.18 & -2.14 & -2.20 & -2.15 & -2.11 & -2.09 & -2.08 & -2.07 & -2.11 & -2.07 & -2.04 & -2.03 & -2.03 & -2.02 \\
\hline & 100 & -2.37 & -2.29 & -2.22 & -2.20 & -2.18 & -2.14 & -2.20 & -2.16 & -2.11 & -2.09 & -2.08 & -2.07 & -2.11 & -2.08 & -2.05 & -2.03 & -2.03 & -2.02 \\
\hline & 200 & -2.35 & -2.30 & -2.23 & -2.20 & -2.18 & -2.15 & -2.20 & -2.16 & -2.12 & -2.10 & -2.08 & -2.07 & -2.11 & -2.08 & -2.05 & -2.04 & -2.03 & -2.02 \\
\hline & 20 & -2.39 & -2.33 & -2.23 & -2.20 & -2.17 & -2.13 & -2.19 & -2.14 & -2.08 & -2.06 & -2.04 & -2.02 & -2.08 & -2.04 & -2.00 & -1.98 & -1.97 & -1.96 \\
\hline & 30 & -2.38 & -2.30 & -2.22 & -2.19 & -2.16 & -2.14 & -2.19 & -2.13 & -2.09 & -2.07 & -2.05 & -2.03 & -2.09 & -2.05 & -2.01 & -2.01 & -1.99 & -1.98 \\
\hline 1 & 50 & -2.37 & -2.28 & -2.21 & -2.19 & -2.16 & -2.14 & -2.20 & -2.14 & -2.10 & -2.08 & -2.07 & -2.05 & -2.10 & -2.06 & -2.03 & -2.02 & -2.00 & -2.00 \\
\hline & 70 & -2.35 & -2.29 & -2.22 & -2.19 & -2.18 & -2.13 & -2.20 & -2.15 & -2.10 & -2.09 & -2.08 & -2.06 & -2.10 & -2.07 & -2.03 & -2.02 & -2.02 & -2.01 \\
\hline & 100 & -2.36 & -2.29 & -2.21 & -2.20 & -2.17 & -2.14 & -2.20 & -2.15 & -2.11 & -2.09 & -2.08 & -2.06 & -2.10 & -2.07 & -2.04 & -2.03 & -2.02 & -2.01 \\
\hline & 200 & -2.35 & -2.30 & -2.23 & -2.20 & -2.18 & -2.15 & -2.19 & -2.16 & -2.12 & -2.10 & -2.08 & -2.07 & -2.11 & -2.08 & -2.05 & -2.04 & -2.03 & -2.02 \\
\hline & 20 & -2.28 & -2.18 & -2.11 & -2.08 & -2.07 & -2.02 & -2.07 & -2.01 & -1.95 & -1.93 & -1.92 & -1.88 & -1.95 & -1.90 & -1.87 & -1.84 & -1.84 & -1.81 \\
\hline & 30 & -2.31 & -2.22 & -2.15 & -2.12 & -2.08 & -2.05 & -2.11 & -2.05 & -2.01 & -1.99 & -1.96 & -1.95 & -2.01 & -1.96 & -1.93 & -1.92 & -1.90 & -1.88 \\
\hline 2 & 50 & -2.32 & -2.23 & -2.17 & -2.15 & -2.12 & -2.09 & -2.15 & -2.10 & -2.05 & -2.03 & -2.02 & -2.00 & -2.05 & -2.01 & -1.98 & -1.96 & -1.95 & -1.94 \\
\hline & 70 & -2.32 & -2.25 & -2.18 & -2.16 & -2.15 & -2.10 & -2.16 & -2.11 & -2.06 & -2.05 & -2.04 & -2.02 & -2.06 & -2.03 & -1.99 & -1.99 & -1.98 & -1.97 \\
\hline & 100 & -2.33 & -2.27 & -2.19 & -2.17 & -2.15 & -2.12 & -2.17 & -2.12 & -2.09 & -2.06 & -2.06 & -2.04 & -2.08 & -2.05 & -2.02 & -2.00 & -2.00 & -1.99 \\
\hline & 200 & -2.34 & -2.29 & -2.21 & -2.19 & -2.16 & -2.13 & -2.18 & -2.15 & -2.10 & -2.09 & -2.07 & -2.06 & -2.10 & -2.06 & -2.04 & -2.02 & -2.01 & -2.01 \\
\hline & 20 & -2.26 & -2.17 & -2.10 & -2.05 & -2.02 & -1.99 & -2.04 & -1.97 & -1.91 & -1.89 & -1.87 & -1.84 & -1.92 & -1.86 & -1.82 & -1.80 & -1.78 & -1.77 \\
\hline & 30 & -2.28 & -2.21 & -2.15 & -2.11 & -2.07 & -2.05 & -2.09 & -2.03 & -1.99 & -1.96 & -1.94 & -1.93 & -1.98 & -1.93 & -1.90 & -1.89 & -1.87 & -1.86 \\
\hline 3 & 50 & -2.31 & -2.24 & -2.17 & -2.14 & -2.11 & -2.09 & -2.14 & -2.08 & -2.04 & -2.02 & -2.00 & -1.99 & -2.04 & -2.00 & -1.96 & -1.95 & -1.94 & -1.93 \\
\hline & 70 & -2.32 & -2.25 & -2.19 & -2.16 & -2.14 & -2.10 & -2.16 & -2.10 & -2.06 & -2.04 & -2.03 & -2.01 & -2.06 & -2.02 & -1.99 & -1.97 & -1.97 & -1.96 \\
\hline & 100 & -2.33 & -2.27 & -2.20 & -2.16 & -2.15 & -2.12 & -2.16 & -2.12 & -2.08 & -2.06 & -2.05 & -2.03 & -2.08 & -2.04 & -2.01 & -2.00 & -1.99 & -1.98 \\
\hline & 200 & -2.34 & -2.29 & -2.20 & -2.19 & -2.16 & -2.13 & -2.18 & -2.15 & -2.10 & -2.08 & -2.07 & -2.05 & -2.09 & -2.06 & -2.03 & -2.02 & -2.01 & -2.00 \\
\hline & 20 & -2.15 & -2.03 & -1.94 & -1.90 & -1.88 & -1.83 & -1.91 & -1.83 & -1.77 & -1.74 & -1.72 & -1.69 & -1.78 & -1.72 & -1.68 & -1.66 & -1.64 & -1.61 \\
\hline & 30 & -2.21 & -2.12 & -2.06 & -2.02 & -1.99 & -1.96 & -2.00 & -1.95 & -1.90 & -1.87 & -1.85 & -1.83 & -1.89 & -1.85 & -1.81 & -1.80 & -1.78 & -1.76 \\
\hline 4 & 50 & -2.27 & -2.19 & -2.13 & -2.10 & -2.06 & -2.04 & -2.09 & -2.03 & -1.99 & -1.97 & -1.95 & -1.94 & -1.99 & -1.95 & -1.91 & -1.90 & -1.88 & -1.88 \\
\hline & 70 & -2.29 & -2.22 & -2.16 & -2.12 & -2.11 & -2.06 & -2.13 & -2.07 & -2.02 & -2.01 & -2.00 & -1.97 & -2.03 & -1.99 & -1.95 & -1.94 & -1.93 & -1.92 \\
\hline & 100 & -2.31 & -2.25 & -2.17 & -2.14 & -2.12 & -2.09 & -2.14 & -2.09 & -2.05 & -2.04 & -2.03 & -2.01 & -2.05 & -2.01 & -1.98 & -1.97 & -1.96 & -1.95 \\
\hline & 200 & -2.33 & -2.27 & -2.20 & -2.18 & -2.15 & -2.12 & -2.17 & -2.13 & -2.09 & -2.07 & -2.05 & -2.04 & -2.08 & -2.05 & -2.02 & -2.01 & -2.00 & -1.99 \\
\hline & & & & & & & & & $k+$ & $1=2$ & & & & & & & & & \\
\hline & & & & $1 \%(\bar{C}$ & $\overline{A D D F})$ & & & & & $5 \%(\bar{C}$ & $\overline{A D F})$ & & & & & $10 \%$ & ADI & & \\
\hline$p$ & $(\mathrm{~T}, \mathrm{~N})$ & 20 & 30 & 50 & 70 & 100 & 200 & 20 & 30 & 50 & 70 & 100 & 200 & 20 & 30 & 50 & 70 & 100 & 200 \\
\hline & 20 & -2.64 & -2.56 & -2.51 & -2.45 & -2.42 & -2.39 & -2.44 & -2.38 & -2.34 & -2.30 & -2.29 & -2.26 & -2.33 & -2.28 & -2.25 & -2.22 & -2.21 & -2.19 \\
\hline & 30 & -2.64 & -2.55 & -2.48 & -2.46 & -2.44 & -2.40 & -2.45 & -2.39 & -2.35 & -2.33 & -2.31 & -2.29 & -2.34 & -2.30 & -2.27 & -2.25 & -2.24 & -2.22 \\
\hline 0 & 50 & -2.62 & -2.56 & -2.49 & -2.46 & -2.44 & -2.42 & -2.45 & -2.40 & -2.36 & -2.34 & -2.32 & -2.30 & -2.35 & -2.32 & -2.28 & -2.27 & -2.26 & -2.25 \\
\hline & 70 & -2.64 & -2.55 & -2.49 & -2.46 & -2.45 & -2.43 & -2.46 & -2.40 & -2.37 & -2.35 & -2.33 & -2.32 & -2.36 & -2.32 & -2.30 & -2.28 & -2.27 & -2.26 \\
\hline & 100 & -2.63 & -2.56 & -2.51 & -2.47 & -2.45 & -2.43 & -2.47 & -2.41 & -2.38 & -2.35 & -2.34 & -2.32 & -2.37 & -2.33 & -2.31 & -2.28 & -2.28 & -2.26 \\
\hline & 200 & -2.63 & -2.56 & -2.50 & -2.48 & -2.46 & -2.43 & -2.46 & -2.42 & -2.38 & -2.36 & -2.35 & -2.33 & -2.37 & -2.34 & -2.31 & -2.29 & -2.29 & -2.27 \\
\hline & 20 & -2.63 & -2.54 & -2.45 & -2.41 & -2.40 & -2.35 & -2.38 & -2.32 & -2.27 & -2.23 & -2.22 & -2.19 & -2.26 & -2.20 & -2.17 & -2.14 & -2.12 & -2.10 \\
\hline & 30 & -2.61 & -2.52 & -2.46 & -2.43 & -2.41 & -2.36 & -2.41 & -2.34 & -2.30 & -2.28 & -2.26 & -2.24 & -2.29 & -2.24 & -2.22 & -2.19 & -2.18 & -2.17 \\
\hline 1 & 50 & -2.62 & -2.55 & -2.48 & -2.44 & -2.43 & -2.40 & -2.43 & -2.37 & -2.33 & -2.31 & -2.30 & -2.28 & -2.33 & -2.29 & -2.25 & -2.24 & -2.23 & -2.21 \\
\hline & 70 & -2.62 & -2.54 & -2.47 & -2.45 & -2.43 & -2.41 & -2.45 & -2.39 & -2.35 & -2.33 & -2.32 & -2.29 & -2.35 & -2.30 & -2.27 & -2.26 & -2.24 & -2.23 \\
\hline & 100 & -2.62 & -2.54 & -2.50 & -2.46 & -2.44 & -2.41 & -2.45 & -2.40 & -2.37 & -2.34 & -2.32 & -2.31 & -2.36 & -2.32 & -2.30 & -2.27 & -2.26 & -2.24 \\
\hline & 200 & -2.62 & -2.55 & -2.50 & -2.47 & -2.46 & -2.43 & -2.46 & -2.41 & -2.37 & -2.36 & -2.34 & -2.32 & -2.37 & -2.33 & -2.30 & -2.28 & -2.28 & -2.26 \\
\hline & 20 & -2.44 & -2.34 & -2.25 & -2.21 & -2.17 & -2.12 & -2.18 & -2.11 & -2.05 & -2.01 & -1.99 & -1.96 & -2.03 & -1.98 & -1.94 & -1.91 & -1.89 & -1.88 \\
\hline & 30 & -2.50 & -2.41 & -2.33 & -2.30 & -2.28 & -2.24 & -2.27 & -2.21 & -2.17 & -2.14 & -2.12 & -2.10 & -2.15 & -2.11 & -2.07 & -2.05 & -2.04 & -2.03 \\
\hline 2 & 50 & -2.55 & -2.49 & -2.40 & -2.37 & -2.35 & -2.32 & -2.35 & -2.31 & -2.25 & -2.24 & -2.22 & -2.20 & -2.25 & -2.22 & -2.17 & -2.16 & -2.15 & -2.13 \\
\hline & 70 & -2.57 & -2.49 & -2.43 & -2.41 & -2.39 & -2.36 & -2.39 & -2.34 & -2.29 & -2.27 & -2.26 & -2.24 & -2.30 & -2.25 & -2.22 & -2.20 & -2.19 & -2.18 \\
\hline & 100 & -2.59 & -2.51 & -2.46 & -2.42 & -2.40 & -2.38 & -2.41 & -2.37 & -2.33 & -2.30 & -2.29 & -2.27 & -2.32 & -2.28 & -2.26 & -2.23 & -2.22 & -2.20 \\
\hline & 200 & -2.61 & -2.54 & -2.48 & -2.46 & -2.43 & -2.40 & -2.43 & -2.39 & -2.35 & -2.34 & -2.33 & -2.30 & -2.35 & -2.31 & -2.28 & -2.26 & -2.27 & -2.24 \\
\hline & 20 & -2.45 & -2.32 & -2.21 & -2.13 & -2.09 & -2.02 & -2.12 & -2.05 & -1.97 & -1.90 & -1.89 & -1.85 & -1.96 & -1.90 & -1.83 & -1.80 & -1.77 & -1.74 \\
\hline & 30 & -2.43 & -2.34 & -2.28 & -2.24 & -2.21 & -2.18 & -2.21 & -2.13 & -2.09 & -2.07 & -2.05 & -2.03 & -2.09 & -2.02 & -1.99 & -1.97 & -1.96 & -1.94 \\
\hline 3 & 50 & -2.53 & -2.45 & -2.38 & -2.36 & -2.33 & -2.30 & -2.32 & -2.27 & -2.22 & -2.20 & -2.19 & -2.17 & -2.21 & -2.18 & -2.13 & -2.12 & -2.11 & -2.09 \\
\hline & 70 & -2.56 & -2.47 & -2.42 & -2.39 & -2.37 & -2.35 & -2.37 & -2.31 & -2.27 & -2.25 & -2.24 & -2.22 & -2.27 & -2.23 & -2.19 & -2.18 & -2.16 & -2.15 \\
\hline & 100 & -2.59 & -2.50 & -2.46 & -2.42 & -2.39 & -2.37 & -2.39 & -2.35 & -2.32 & -2.29 & -2.27 & -2.25 & -2.30 & -2.26 & -2.24 & -2.21 & -2.20 & -2.19 \\
\hline & 200 & -2.61 & -2.54 & -2.47 & -2.45 & -2.43 & -2.40 & -2.43 & -2.39 & -2.34 & -2.33 & -2.32 & -2.30 & -2.34 & -2.31 & -2.27 & -2.26 & -2.26 & -2.24 \\
\hline & 20 & - & - & - & - & - & - & - & - & - & - & - & - & - & - & - & - & - & - \\
\hline & 30 & & -2.20 & -2.12 & -2.06 & -2.05 & -2.03 & -2.05 & -1.99 & -1.94 & -1.91 & -1.89 & -1.86 & -1.93 & -1.87 & -1.84 & -1.81 & -1.79 & -1.78 \\
\hline 4 & 50 & -2.44 & -2.37 & -2.30 & -2.28 & -2.23 & -2.21 & -2.24 & -2.19 & -2.14 & -2.12 & -2.11 & -2.08 & -2.13 & -2.09 & -2.05 & -2.04 & -2.02 & -2.01 \\
\hline & 70 & -2.52 & -2.43 & -2.37 & -2.34 & -2.32 & -2.30 & -2.32 & -2.26 & -2.22 & -2.20 & -2.19 & -2.17 & -2.22 & -2.17 & -2.14 & -2.12 & -2.11 & -2.10 \\
\hline & 100 & -2.54 & -2.48 & -2.42 & -2.38 & -2.35 & -2.33 & -2.36 & -2.31 & -2.28 & -2.25 & -2.23 & -2.22 & -2.26 & -2.23 & -2.20 & -2.17 & -2.17 & -2.15 \\
\hline & 200 & -2.60 & -2.52 & -2.46 & -2.44 & -2.42 & -2.39 & -2.41 & -2.37 & -2.33 & -2.30 & -2.30 & -2.28 & -2.32 & -2.29 & -2.26 & -2.24 & -2.24 & -2.22 \\
\hline
\end{tabular}




\begin{tabular}{|c|c|c|c|c|c|c|c|c|c|c|c|c|c|c|c|c|c|c|c|}
\hline & & & & & & & & & & & & & & & & & & & \\
\hline & & & & & & & & & $k+$ & $-1=3$ & & & & & & & & & \\
\hline & & & & $1 \%(\bar{C}$ & $\overline{A D F}$ & & & & & $5 \%(\bar{C}$ & $A D F$ & & & & & $10 \%(\bar{C}$ & $\overline{\overline{C A D F}}$ & & \\
\hline$p$ & $(\mathrm{~T}, \mathrm{~N})$ & 20 & 30 & 50 & 70 & 100 & 200 & 20 & 30 & 50 & 70 & 100 & 200 & 20 & 30 & 50 & 70 & 100 & 200 \\
\hline & 20 & -2.84 & -2.78 & -2.68 & -2.65 & -2.59 & -2.57 & -2.60 & -2.56 & -2.49 & -2.48 & -2.45 & -2.43 & -2.48 & -2.45 & -2.40 & -2.39 & -2.37 & -2.35 \\
\hline & 30 & -2.84 & -2.76 & -2.68 & -2.65 & -2.63 & -2.59 & -2.64 & -2.58 & -2.53 & -2.51 & -2.50 & -2.47 & -2.53 & -2.49 & -2.45 & -2.43 & -2.42 & -2.40 \\
\hline 0 & 50 & -2.84 & -2.77 & -2.70 & -2.67 & -2.66 & -2.62 & -2.66 & -2.61 & -2.56 & -2.55 & -2.53 & -2.51 & -2.57 & -2.52 & -2.48 & -2.48 & -2.46 & -2.45 \\
\hline & 70 & -2.86 & -2.78 & -2.72 & -2.68 & -2.67 & -2.64 & -2.67 & -2.62 & -2.58 & -2.56 & -2.55 & -2.53 & -2.58 & -2.54 & -2.50 & -2.49 & -2.48 & -2.47 \\
\hline & 100 & -2.85 & -2.79 & -2.72 & -2.69 & -2.67 & -2.64 & -2.68 & -2.64 & -2.59 & -2.57 & -2.56 & -2.54 & -2.59 & -2.56 & -2.52 & -2.50 & -2.49 & -2.48 \\
\hline & 200 & -2.87 & -2.80 & -2.74 & -2.70 & -2.68 & -2.66 & -2.69 & -2.65 & -2.61 & -2.58 & -2.56 & -2.55 & -2.60 & -2.57 & -2.53 & -2.52 & -2.50 & -2.49 \\
\hline & 20 & -2.78 & -2.68 & -2.55 & -2.53 & -2.48 & -2.46 & -2.47 & -2.40 & -2.33 & -2.32 & -2.30 & -2.27 & -2.33 & -2.27 & -2.23 & -2.21 & -2.19 & -2.17 \\
\hline & 30 & -2.76 & -2.69 & -2.61 & -2.57 & -2.56 & -2.51 & -2.54 & -2.49 & -2.43 & -2.41 & -2.39 & -2.36 & -2.42 & -2.38 & -2.34 & -2.32 & -2.31 & -2.29 \\
\hline 1 & 50 & -2.80 & -2.74 & -2.67 & -2.64 & -2.61 & -2.58 & -2.61 & -2.56 & -2.51 & -2.50 & -2.47 & -2.45 & -2.50 & -2.47 & -2.43 & -2.42 & -2.40 & -2.38 \\
\hline & 70 & -2.83 & -2.75 & -2.68 & -2.65 & -2.64 & -2.60 & -2.65 & -2.59 & -2.53 & -2.52 & -2.51 & -2.49 & -2.54 & -2.49 & -2.46 & -2.44 & -2.44 & -2.42 \\
\hline & 100 & -2.84 & -2.78 & -2.70 & -2.67 & -2.65 & -2.62 & -2.65 & -2.61 & -2.57 & -2.54 & -2.53 & -2.51 & -2.56 & -2.52 & -2.49 & -2.47 & -2.46 & -2.45 \\
\hline & 200 & -2.85 & -2.80 & -2.72 & -2.69 & -2.67 & -2.65 & -2.69 & -2.64 & -2.60 & -2.57 & -2.56 & -2.54 & -2.59 & -2.56 & -2.52 & -2.50 & -2.49 & -2.48 \\
\hline & 20 & -2.71 & -2.51 & -2.33 & -2.27 & -2.21 & -2.16 & \begin{tabular}{|l|} 
\\
\end{tabular} & -2.17 & -2.08 & -2.03 & -1.99 & -1.95 & -2.08 & -2.01 & -1.93 & -1.90 & -1.87 & -1.84 \\
\hline & 30 & -2.58 & -2.50 & -2.41 & -2.36 & -2.34 & -2.30 & -2.35 & -2.30 & -2.22 & -2.20 & -2.18 & -2.15 & -2.21 & -2.17 & -2.12 & -2.10 & -2.10 & -2.07 \\
\hline 2 & 50 & -2.70 & -2.63 & -2.55 & -2.53 & -2.50 & -2.47 & -2.50 & -2.45 & -2.39 & -2.39 & -2.37 & -2.34 & -2.38 & -2.35 & -2.30 & -2.30 & -2.28 & -2.26 \\
\hline & 70 & .75 & -2.68 & -2.61 & -2.58 & -2.57 & -2.53 & -2.57 & -2.51 & -2.46 & -2.44 & -2.44 & -2 & -2.45 & -2.42 & -2.38 & -2.37 & -2.36 & -2.34 \\
\hline & 100 & -2.79 & -2.72 & -2.65 & -2.62 & -2.60 & -2.57 & -2.60 & -2.56 & -2.51 & -2.49 & -2.47 & -2. & -2.51 & -2.46 & -2.44 & -2.42 & -2.40 & -2.39 \\
\hline & 200 & -2.84 & -2.77 & -2.69 & -2.66 & -2.65 & -2.62 & -2.67 & -2.62 & -2.57 & -2.54 & -2.53 & -2.51 & -2.56 & -2.53 & -2.49 & -2.48 & -2.46 & -2.45 \\
\hline & 20 & & & & & & & & & & & & & & & & & & \\
\hline & 30 & 2.47 & -2.37 & -2.27 & -2.21 & -2.19 & -2.16 & -2.20 & -2.14 & -2.07 & -2.04 & -2.02 & -2.00 & -2.07 & -2.02 & -1.96 & -1.94 & -1.93 & -1.90 \\
\hline 3 & 50 & - & -2.57 & -2.49 & -2.48 & -2.44 & -2.4 & -2 & -2.38 & -2.32 & -2.31 & -2.2 & & -2 & & -2 & -2.23 & -2.21 & -2.19 \\
\hline & 70 & .72 & -2.65 & -2.56 & -2.54 & -2.53 & -2.49 & -2. & -2.47 & -2.42 & -2.40 & -2.39 & -2 . & -2.41 & -2.37 & -2.33 & -2.32 & -2.31 & -2.30 \\
\hline & 100 & -2.77 & -2.69 & -2.63 & -2.60 & -2.58 & -2.54 & -2.58 & -2.53 & -2.49 & -2.46 & -2.45 & -2 . & -2 & -2.44 & -2.41 & -2.39 & -2.37 & -2.36 \\
\hline & 200 & -2.82 & -2.76 & -2.68 & -2.65 & -2.63 & -2.61 & -2.65 & -2.61 & -2.55 & -2.53 & -2.52 & -2.50 & -2.55 & -2.52 & -2.48 & -2.46 & -2.44 & -2.44 \\
\hline & 20 & & & - & - & - & & & & & & & & & & & & - & \\
\hline & 30 & -2.28 & -2.15 & -2.04 & -2.01 & -1.97 & -1.89 & -2. & -1.90 & -1.83 & -1.81 & -1.78 & -1.72 & -1.85 & -1.77 & -1 & -1.70 & -1.68 & -1.64 \\
\hline 4 & 50 & .53 & -2.45 & -2.38 & -2.35 & -2.31 & -2.28 & -2.31 & -2.25 & -2.20 & -2.19 & -2.16 & -2 . & -2.19 & -2.15 & -2 & -2.10 & -2.08 & -2.06 \\
\hline & 70 & -2.64 & -2.59 & -2.50 & -2.48 & -2.45 & -2.41 & -2.45 & -2.38 & -2.34 & -2.32 & -2.31 & -2. & -2.33 & -2.29 & -2.25 & -2.24 & -2.23 & -2.21 \\
\hline & 100 & -2.71 & -2.65 & -2.58 & -2.55 & -2.53 & -2.49 & -2.5 & -2.47 & -2.44 & -2.41 & -2.39 & -2 . & -2.43 & -2.38 & -2 & -2.33 & -2.32 & -2.30 \\
\hline & 200 & -2.80 & -2.74 & -2.66 & -2.63 & -2.60 & -2.58 & -2.62 & -2.58 & -2.53 & -2.51 & -2.49 & -2.47 & -2.52 & -2.49 & -2.45 & -2.43 & -2.42 & -2.41 \\
\hline & & & & & & & & & $k+$ & $-1=4$ & & & & & & & & & \\
\hline & & & & $1 \%(\bar{C}$ & $\overline{A D F}$ & & & & & $5 \%(\bar{C}$ & $A D F$ & & & & & $10 \%(\bar{C}$ & $\overline{C A D F}$ & & \\
\hline$p$ & $(\mathrm{~T}, \mathrm{~N})$ & 20 & 30 & 50 & 70 & 100 & 200 & 20 & 30 & 50 & 70 & 100 & 200 & 20 & 30 & 50 & 70 & 100 & 200 \\
\hline & 20 & -2.99 & -2.91 & -2.82 & -2.76 & -2.75 & -2.71 & -2.73 & -2.68 & -2.63 & -2.58 & -2.57 & -2. & & -2.56 & -2.52 & -2.49 & -2.48 & -2.45 \\
\hline & 30 & -3.00 & $\begin{array}{l}-2.89 \\
\end{array}$ & -2.85 & -2.81 & $\begin{array}{l}-2.78 \\
\end{array}$ & -2.73 & $\begin{array}{l}-2.79 \\
\end{array}$ & $\begin{array}{l}-2.73 \\
\end{array}$ & -2.69 & -2.66 & -2.64 & -2.6 & -2.68 & -2.63 & $\begin{array}{l}-2.60 \\
\end{array}$ & -2.57 & $\begin{array}{l}-2.56 \\
\end{array}$ & -2.54 \\
\hline 0 & 50 & & -2.95 & -2.88 & -2.86 & -2.82 & -2.80 & -2.83 & -2.79 & -2.74 & -2.72 & -2.70 & & & & -2 . & -2.64 & -2.63 & -2.62 \\
\hline & 70 & .04 & $\begin{array}{l}-2.97 \\
\end{array}$ & -2.89 & -2.86 & -2.84 & -2.82 & $\begin{array}{l}-2.86 \\
\end{array}$ & $\begin{array}{l}-2.81 \\
\end{array}$ & -2.77 & $\begin{array}{l}-2.74 \\
\end{array}$ & -2.72 & -2 . & -2.76 & -2.73 & -2. & -2.67 & $\begin{array}{l}-2.66 \\
\end{array}$ & -2.65 \\
\hline & 100 & & -2.97 & -2.92 & -2.88 & -2.86 & -2.8 & -2 & -2.82 & -2.78 & -2 & -2 & & -2 & & -2 & -2 & -2.68 & -2.66 \\
\hline & 200 & -3.07 & -2.99 & -2.92 & -2.90 & -2.87 & -2.85 & -2.89 & -2.84 & -2.80 & -2.79 & -2.76 & -2 & -2.80 & -2.76 & -2 & -2.71 & -2.70 & -2.68 \\
\hline & 20 & -2.91 & -2.77 & -2.66 & -2.58 & -2.52 & -2.47 & -2.55 & -2.47 & -2.39 & -2.34 & -2.30 & -2.26 & -2.37 & -2.30 & -2.25 & -2.20 & -2.19 & -2.15 \\
\hline & 30 & -2.86 & -2.77 & -2.69 & -2.67 & -2.62 & -2.59 & -2 . & -2.56 & -2.52 & -2 & -2.4 & & -2 . & & -2 . & -2 & & -2.35 \\
\hline 1 & 50 & -2.94 & -2.88 & -2.80 & -2.78 & -2.74 & -2.72 & -2.75 & -2.69 & -2.65 & -2.62 & -2.61 & -2.59 & -2.65 & -2.60 & -2.56 & -2.54 & -2.53 & -2.52 \\
\hline & 70 & & -2.90 & -2.85 & -2.82 & -2.79 & -2.77 & -2. & -2.75 & -2.70 & -2. & -2.6 & & -2 . & & -2 . & -2 . & -2.59 & -2.58 \\
\hline & 100 & -3.01 & -2.93 & -2.87 & -2.84 & -2.83 & -2.80 & -2.83 & -2.78 & -2.74 & -2.72 & -2.70 & -2.68 & -2.73 & -2.70 & -2.66 & -2.64 & -2.63 & -2.61 \\
\hline & 200 & -3. & -2.96 & -2.90 & -2.89 & -2.86 & -2.82 & -2. & -2.82 & -2.77 & -2.76 & -2.74 & -2 . & -2 & -2.74 & -2 & -2.69 & -2.68 & -2.66 \\
\hline & 20 & & & & & & & & - & & & & & & & & & - & \\
\hline & 30 & & -2.47 & -2.39 & -2.35 & -2.31 & -2.27 & & -2.27 & -2.20 & & & & & & & & & \\
\hline 2 & 50 & -2.8 & -2.74 & -2.65 & -2.63 & -2.58 & -2.58 & -2.60 & -2.55 & -2.50 & -2.46 & -2.45 & -2.44 & -2.48 & -2.45 & -2 & -2.38 & -2.37 & -2.35 \\
\hline & 70 & & -2.81 & -2.76 & -2.72 & -2.69 & -2.6 & & -2.65 & -2.60 & -2 & & & & & & -2.49 & -2.49 & -2.47 \\
\hline & 100 & -2.9 & -2.88 & -2.81 & -2.78 & -2.75 & -2.72 & -2.7 & -2.71 & -2.67 & -2.64 & -2.63 & -2. & -2 & -2.62 & -2 & -2.57 & -2.55 & -2.54 \\
\hline & 200 & -3.01 & -2.94 & -2.87 & -2.85 & -2.83 & -2.79 & -2.84 & -2.79 & -2.74 & -2.73 & -2.71 & -2.69 & -2.75 & -2.70 & -2.66 & -2.66 & -2.65 & -2.63 \\
\hline & 20 & & & & & & & & & & & & & & & & & & \\
\hline & 30 & -2 & -2.35 & -2.19 & -2.1 & -2.09 & -2.0 & & & & & & & & & & & & -1. \\
\hline 3 & 50 & -2.7 & $\begin{array}{l}-2.62 \\
\end{array}$ & -2.54 & -2.52 & -2.48 & -2.4 & -2 . & -2.43 & -2.37 & -2 . & -2. & & -2 . & & -2 . & -2.26 & -2.25 & -2.23 \\
\hline & 70 & & -2.77 & -2.71 & -2.66 & -2.63 & -2.60 & -2 . & -2.57 & -2 & -2 . & -2. & & -2 & & & -2.41 & -2.41 & -2.39 \\
\hline & 100 & -2.9 & -2.83 & -2.76 & -2.73 & -2.71 & -2.68 & -2 & -2.67 & -2.62 & -2 . & -2. & -2 . & -2 & -2 & -2 . & -2.52 & -2.51 & -2.49 \\
\hline & 200 & -3.00 & -2.94 & -2.85 & -2.84 & -2.82 & -2.78 & -2.82 & -2.77 & -2.72 & -2.71 & -2.69 & -2.67 & -2.72 & -2.68 & -2.64 & -2.64 & -2.62 & -2.61 \\
\hline & & & & & 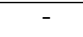 & - & & & & & & & - & - & & $\begin{array}{lll}- & \text { - }\end{array}$ & & & - \\
\hline & 30 & - & - & - & & - & - & - & - & - & - & - & & & & - & - & & - \\
\hline 4 & 50 & -2.52 & -2.44 & -2.36 & -2.33 & -2.30 & -2.28 & -2.32 & -2.24 & -2.19 & -2.16 & -2.14 & -2.1 & -2.19 & -2.13 & -2.0 & -2.07 & -2.06 & -2.03 \\
\hline & 70 & & -2.65 & -2.58 & -2.55 & -2.52 & -2.50 & -2. & -2.46 & -2.41 & -2.39 & -2.37 & -2. & -2.40 & -2 & -2 & -2.29 & -2.29 & -2.27 \\
\hline & 100 & -2.84 & -2.78 & -2.71 & -2.67 & -2.65 & -2.61 & -2.65 & -2.60 & -2.54 & -2.52 & -2.51 & -2.48 & -2.54 & -2.50 & -2.46 & -2.44 & -2.43 & -2.41 \\
\hline & 200 & -2.98 & -2.90 & -2.82 & -2.81 & -2.78 & -2.74 & -2.78 & -2.73 & -2.68 & -2.68 & -2.66 & -2.63 & -2.69 & -2.64 & -2.61 & -2.60 & -2.59 & -2.57 \\
\hline
\end{tabular}

Notes: The critical values are obtained by stochastic simulation. The data generating process is $y_{i t}=y_{i t-1}+f_{t}+u_{i t}$ with $f_{t} \sim \operatorname{iidN}(0,1), u_{i t} \sim \operatorname{iidN}(0,1)$, with $y_{i 0}=0$, and the $j^{\text {th }}$ element of the $k \times 1$ vector of additional regressors, $x_{i t}$, are generated as $x_{i j t}=x_{i j t-1}+f_{j t}+v_{i j t}$, with $f_{j t} \sim i i d N(0,1), v_{i j t} \sim i i d N(0,1)$ and $x_{i j 0}=0, i=1,2, \ldots, N ; j=1,2, \ldots, k$; $t=-p, \ldots, T$. Based on these data, $C A D F_{i}$, which is the $t$-ratio of coefficient on $y_{i t-1}$ of regression of $\Delta y_{i t}$ on $y_{i t-1}$, 
$w_{i t, p}^{\prime}=\left(\bar{z}_{t-1}^{\prime} ; \Delta \bar{z}_{t}^{\prime}, \Delta \bar{z}_{t-1}^{\prime}, \ldots, \Delta \bar{z}_{t-p}^{\prime} ; \Delta y_{i, t-1}, \ldots, \Delta y_{i, t-p}\right)$ with $\bar{z}_{t}=N^{-1} \sum_{i=1}^{N}\left(y_{i t}, x_{i t}^{\prime}\right)^{\prime}$ and deterministics as specified, then $\overline{C A D F}=N^{-1} \sum_{i=1}^{N} C A D F_{i}$ are computed. $(100 \times \alpha) \%$ critical values are obtained as the $1-\alpha$ quantiles of $\overline{C A D F}$ for $\alpha=0.01,0.05,0.1$. Computations are based on 10000 replications. 
Table 1c: Critical Values of Average of Individual Cross-Sectionally Augmented Dickey-Fuller Distribution (Case III: Intercept and Trend)

\begin{tabular}{|c|c|c|c|c|c|c|c|c|c|c|c|c|c|c|c|c|c|c|c|}
\hline \multicolumn{20}{|c|}{$k+1=1$} \\
\hline \multirow[b]{2}{*}{$p$} & \multirow[b]{2}{*}{$(\mathrm{T}, \mathrm{N})$} & \multicolumn{6}{|c|}{ 1\% $(\overline{C A D F})$} & \multicolumn{6}{|c|}{$\mathbf{5 \%}(\overline{C A D F})$} & \multicolumn{6}{|c|}{$10 \%(\overline{C A D F})$} \\
\hline & & 20 & 30 & 50 & 70 & 100 & 200 & 20 & 30 & 50 & 70 & 100 & 200 & 20 & 30 & 50 & 70 & 100 & 200 \\
\hline & 20 & 2.92 & -2.84 & -2.76 & -2.72 & -2.69 & -2.65 & -2.73 & -2.68 & -2.62 & -2.60 & -2.57 & -2.55 & -2.63 & -2.59 & -2.54 & -2.52 & -2.51 & -2.49 \\
\hline & 30 & 2.88 & -2.79 & -2.73 & -2.69 & -2.66 & -2.62 & 2.71 & -2.66 & -2.61 & -2.58 & -2.56 & -2.54 & -2.62 & -2.58 & -2.55 & -2.53 & -2.51 & -2.49 \\
\hline 0 & 50 & -2.86 & -2.79 & -2.70 & -2.69 & -2.66 & -2.62 & 2.70 & -2.66 & -2.61 & -2.59 & -2.57 & -2.55 & -2.62 & -2.59 & -2.55 & -2.53 & -2.52 & -2.50 \\
\hline & 70 & 2.87 & -2.78 & -2.71 & -2.68 & -2.65 & -2.62 & 2.70 & 2.65 & -2.61 & -2.59 & -2.56 & -2.54 & -2.63 & -2.59 & -2.55 & -2.54 & -2.51 & -2.50 \\
\hline & 100 & 2.84 & -2.78 & -2.71 & -2.68 & -2.66 & -2.62 & 2.70 & 2.65 & -2.61 & -2.59 & -2.57 & -2.54 & -2.63 & -2.59 & -2.55 & -2.53 & -2.52 & -2.50 \\
\hline & 200 & & -2.76 & -2.71 & -2.67 & -2.65 & -2.61 & 2.70 & 2.65 & -2.61 & & -2.57 & & & -2.59 & -2.55 & -2.53 & -2.52 & -2.50 \\
\hline & 20 & 2.96 & -2.87 & -2.80 & -2.74 & -2.71 & -2.69 & -2.73 & 2.68 & -2.63 & -2.60 & -2.58 & -2.55 & -2.62 & -2.57 & -2.53 & -2.51 & -2.49 & -2.47 \\
\hline & 30 & 2.90 & -2.81 & -2.74 & -2.70 & -2.68 & -2.64 & -2.71 & 2.66 & -2.61 & -2.58 & -2.56 & -2.54 & -2.61 & -2.57 & -2.53 & -2.52 & -2.50 & -2.48 \\
\hline 1 & 50 & -2.87 & -2.80 & -2.71 & -2.69 & -2.67 & -2.63 & 2.70 & -2.66 & -2.61 & -2.59 & -2.57 & -2.54 & -2.62 & -2.58 & -2.54 & -2.52 & -2.51 & -2.49 \\
\hline & 70 & 2.87 & -2.79 & -2.71 & -2.68 & -2.65 & -2.62 & 2.71 & 2.65 & -2.60 & -2.58 & -2.56 & -2.54 & -2.62 & -2.58 & -2.54 & -2.53 & -2.51 & -2.50 \\
\hline & 100 & 2.83 & -2.79 & -2.71 & -2.68 & -2.66 & -2.62 & 2.70 & 2.65 & -2.61 & -2.58 & -2.57 & -2.54 & -2.62 & -2.59 & -2.55 & -2.53 & -2.52 & -2.50 \\
\hline & 200 & 2.83 & -2.77 & -2.71 & -2.68 & -2.65 & -2.61 & 2.70 & -2.65 & -2.61 & -2.58 & -2.57 & -2.54 & -2.62 & -2.59 & -2.55 & -2.53 & -2.52 & -2.50 \\
\hline & 2 & 2.80 & -2.71 & -2.61 & -2.58 & -2.56 & -2.53 & -2.56 & -2.50 & & -2.42 & -2.41 & -2.38 & -2.44 & -2.39 & -2.36 & -2.33 & -2.33 & -2.29 \\
\hline & 30 & & -2.72 & -2.65 & -2.62 & -2.60 & -2.56 & -2.62 & -2.56 & -2.51 & -2.49 & -2.47 & -2.44 & & -2.46 & -2.43 & -2.41 & -2.39 & -2.38 \\
\hline 2 & 50 & .82 & -2.75 & -2.67 & -2.64 & -2.62 & -2.58 & 2.65 & 2.60 & -2.55 & -2 . & -2.51 & & & & -2.48 & -2.47 & -2.45 & -2.44 \\
\hline & 70 & 2.82 & -2.76 & -2.67 & -2.65 & -2.61 & -2.59 & 2.66 & -2.61 & -2.57 & -2.55 & -2.53 & -2 . & & -2.54 & -2.50 & -2.49 & -2.47 & -2.46 \\
\hline & 100 & 2.81 & -2.76 & -2.68 & -2.66 & -2.63 & -2.59 & 2.67 & -2.62 & -2.58 & -2.56 & -2.54 & -2.52 & -2.59 & -2.55 & -2.52 & -2.50 & -2.49 & -2.47 \\
\hline & 200 & -2.82 & -2.76 & -2.70 & -2.66 & -2.64 & -2.60 & 2.69 & -2.64 & -2.59 & -2.57 & -2.56 & -2.53 & -2.61 & -2.57 & -2.54 & -2.52 & -2.51 & -2.49 \\
\hline & & -2.78 & -2.68 & -2.58 & -2.54 & -2.51 & -2.47 & -2.52 & -2.46 & -2.40 & -2.37 & -2.35 & -2.32 & -2.39 & -2.34 & -2.29 & -2.28 & -2.25 & -2.23 \\
\hline & 30 & 2.80 & -2.72 & -2.64 & -2.62 & -2.58 & -2.55 & -2.60 & -2.54 & -2.48 & -2.47 & -2.45 & -2 & 48 & -2.43 & -2.40 & -2.38 & -2.37 & -2.35 \\
\hline 3 & 50 & 81 & -2.76 & -2.68 & -2.64 & -2.62 & -2. & 2.64 & -2.59 & -2.54 & -2 . & & & & -2 . & -2.47 & -2.45 & -2.44 & -2.42 \\
\hline & 70 & & -2.75 & -2.68 & -2.66 & -2.61 & -2 . & & -2.61 & -2.57 & & & & & & & & & -2.46 \\
\hline & & & -2.76 & -2.69 & -2.66 & -2.63 & 0 & & 2 & -2 & & & & & & & & & -2.47 \\
\hline & 200 & 2.82 & -2.76 & -2.70 & -2.66 & -2.63 & -2.60 & 2.68 & -2.64 & -2.60 & -2.57 & -2.55 & -2.53 & -2.60 & -2.57 & -2.54 & -2.52 & -2.51 & -2.49 \\
\hline & 20 & 2.60 & -2.52 & -2.39 & -2.35 & -2.31 & -2.26 & -2.35 & -2.26 & -2.19 & -2.17 & -2.15 & -2.11 & -2.21 & -2.15 & -2.09 & -2.07 & -2.06 & -2.02 \\
\hline & 30 & .68 & -2.60 & -2.53 & -2.50 & -2.47 & -2.43 & -2.47 & -2.42 & -2.37 & -2.35 & -2.33 & -2.30 & & -2.31 & -2.28 & -2.26 & -2.25 & -2.22 \\
\hline 4 & 50 & & -2.69 & -2.62 & -2.58 & -2.57 & -2.52 & -2.57 & -2.53 & -2.49 & & & & & & -2 . & -2.39 & & -2.36 \\
\hline & 70 & .78 & -2.72 & -2.64 & -2.62 & -2.58 & -2 . & -2 . & -2.5 & -2. & -2 . & -2. & & & -2 . & & & & -2.41 \\
\hline & & & -2.72 & -2.67 & -2.6 & -2.6 & & -2 . & -2.60 & -2 . & & & & & & & & & -2.44 \\
\hline & & & 2.74 & -2.68 & -2.65 & 2 & & 2 & -2.62 & -2.58 & -2 & & & & & & & & \\
\hline & & & & & & & & & & $1=2$ & & & & & & & & & \\
\hline & & & & 170 & & & & & & $5 \%(\bar{C}$ & & & & & & 07 & & & \\
\hline$p$ & $(\mathrm{~T}, \mathrm{~N})$ & 20 & 30 & 50 & 70 & 100 & 200 & 20 & 30 & 50 & 70 & 100 & 200 & 20 & 30 & 50 & 70 & 100 & 200 \\
\hline & 20 & 3.12 & -3.02 & -2.94 & -2.91 & -2.86 & -2.82 & -2.90 & -2.85 & -2.78 & -2.75 & -2.73 & -2.70 & -2.78 & -2.74 & -2.70 & -2.67 & -2.65 & -2.63 \\
\hline & 30 & 3.07 & -2.99 & -2.92 & -2.89 & -2.86 & -2.82 & 2.89 & -2.84 & -2.78 & -2.76 & -2.74 & -2 . & -2.79 & -2.75 & -2.71 & -2.69 & -2.68 & -2.66 \\
\hline 0 & 50 & & -2.99 & -2.93 & -2.88 & -2.88 & -2.83 & -2.89 & -2.85 & -2.81 & -2.7 & -2.76 & -2 . & & -2.77 & -2 . & -2.7 & -2.70 & -2.68 \\
\hline & 7 & & & -2.93 & -2.8 & -2.8 & -2 . & 1 & -2. & -2 & & & & & & -2 & -2 . & & -2.69 \\
\hline & & & .00 & -2.92 & -2.90 & -2.88 & 2. & 1 & -2.8 & -2 & -2 . & -2 . & -2 . & & -2 . & -2 . & -2 . & -2 & -2.70 \\
\hline & 200 & & 2.99 & -2.94 & -2.91 & -2.88 & 2.84 & 2.91 & -2.86 & -2.82 & -2.79 & -2.78 & -2 & 2.83 & -2.79 & -2.75 & -2.74 & -2.72 & -2.70 \\
\hline & & & -2.99 & -2.90 & -2.88 & -2.85 & -2.81 & 2.83 & -2.76 & -2.72 & -2.67 & -2.65 & & 2.69 & -2.64 & -2.60 & -2.57 & -2.56 & -2.53 \\
\hline & 30 & & -2.96 & -2.89 & -2.88 & -2.83 & -2.8 & 2.85 & -2.79 & -2.74 & -2.71 & -2 . & & & -2.69 & -2.65 & -2.63 & -2.62 & -2.60 \\
\hline 1 & 50 & & -2.96 & -2.90 & -2.87 & -2.86 & -2.82 & -2.87 & -2.82 & -2.78 & -2.74 & -2.73 & & & -2.73 & -2.70 & -2.67 & -2.67 & -2.65 \\
\hline & 7 & & -2.99 & -2.90 & -2.88 & -2.84 & -2.82 & -2.89 & -2.83 & -2.78 & -2.76 & -2.75 & & & -2.75 & -2.72 & -2.70 & -2.69 & -2.67 \\
\hline & & & -2.99 & -2.91 & -2.89 & -2.87 & & & -2.85 & & & & & & & -2 . & -2 . & -2.70 & -2.68 \\
\hline & & & 2.99 & -2.93 & -2.90 & -2.87 & -2 & 0 & -2.8 & -2. & -2.7 & -2. & & -2.82 & -2.78 & -2.75 & -2.73 & -2.71 & -2.70 \\
\hline & & & 2.74 & -2.64 & -2.60 & -2.56 & & 7 & -2.4 & -2 . & -2 & -2 & & & -2 . & -2 . & -2.27 & -2.26 & -2.24 \\
\hline & & & -2.81 & -2.75 & -2.72 & -2.68 & -2.6 & & -2.62 & & -2. & -2.53 & & & & & & -2.45 & -2.43 \\
\hline 2 & 50 & & -2.89 & -2.83 & -2.79 & -2.78 & -2.7 & -2.78 & -2.73 & -2.70 & -2 . & & & & -2.64 & -2 & & -2.57 & -2.55 \\
\hline & & & -2.94 & -2.84 & -2.83 & -2.80 & -2.77 & -2.83 & -2.77 & -2.72 & -2.71 & -2.69 & & & -2.69 & -2.66 & & -2.62 & -2.61 \\
\hline & & & -2.96 & -2.88 & -2.85 & -2.83 & -2.7 & & -2.81 & & & & & & -2.72 & -2.6 & -2.67 & -2.65 & -2.64 \\
\hline & & & & -2.91 & -2.88 & -2.85 & -2.8 & & -2 & -2 . & -2 & & & & -2.76 & -2.73 & -2.71 & -2.70 & -2.68 \\
\hline & & & & & & & & & & & & & & & -2 . & -2 . & -2 & -2.15 & -2.11 \\
\hline & & & & & & -2.62 & & & & & & & & & & & & & -2.33 \\
\hline 3 & & & -2.86 & -2.81 & -2.75 & -2.74 & -2 . & & -2 . & -2 . & -2 & -2 & & & & & & & -2.51 \\
\hline & & & -2.94 & -2.83 & -2.81 & -2.78 & & & -2.74 & -2 . & -2 . & & & & & & & & -2.58 \\
\hline & 100 & & -2.94 & -2.86 & -2.84 & -2.82 & -2 & & -2.79 & -2 . & -2 . & & & & -2 . & -2 . & & -2.64 & -2.62 \\
\hline & & 3.03 & -2.96 & -2.90 & -2.87 & -2.85 & -2.81 & -2.88 & -2.82 & -2.78 & -2.76 & -2.75 & -2.72 & 2.78 & -2.75 & -2.72 & -2.70 & -2.69 & -2.67 \\
\hline & & & - & - & - & & & & - & - & - & & & & & - & - & 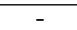 & - \\
\hline & & & -2.56 & & & & & & & & & & & & & & & & -2.12 \\
\hline 4 & 50 & & -2.76 & -2.70 & -2.67 & -2.65 & -2.62 & & -2.59 & -2.56 & & & & & & -2.46 & -2.44 & & -2.40 \\
\hline & 70 & 6.90 & -2.86 & -2.77 & -2.75 & -2.72 & -2.70 & -2.75 & -2.68 & -2.64 & -2.6 & & & & & -2.56 & & -2.53 & -2.52 \\
\hline & 100 & .90 & -2.91 & -2.83 & -2.81 & -2.78 & -2.74 & -2.7 & -2.75 & -2.70 & -2 & -2.66 & -2 & & -2.66 & -2.62 & -2.61 & -2.59 & -2.58 \\
\hline & 200 & .02 & -2.94 & -2.88 & -2.85 & -2.83 & -2.79 & -2.86 & -2.81 & -2.76 & -2.74 & -2.73 & -2.70 & -2.77 & -2.73 & -2.70 & -2.68 & -2.67 & -2.65 \\
\hline
\end{tabular}




\begin{tabular}{|c|c|c|c|c|c|c|c|c|c|c|c|c|c|c|c|c|c|c|c|}
\hline & & & & & & & & & & & & & & & & & & Orumu & \\
\hline & & & & & & & & & $k+$ & $-1=3$ & & & & & & & & & \\
\hline & & & & $1 \%(\bar{C}$ & $A D F$ & & & & & $5 \%(\bar{C}$ & $A D F$ & & & & & $10 \%(\bar{C}$ & $\overline{\overline{C A D F}}$ & & \\
\hline$p$ & $(\mathrm{~T}, \mathrm{~N})$ & 20 & 30 & 50 & 70 & 100 & 200 & 20 & 30 & 50 & 70 & 100 & 200 & 20 & 30 & 50 & 70 & 100 & 200 \\
\hline & 20 & -3.26 & -3.15 & -3.09 & -3.04 & -3.00 & -2.97 & \begin{tabular}{|l|}
-3.01 \\
\end{tabular} & -2.95 & -2.89 & -2.86 & -2.84 & -2.82 & -2.89 & -2.84 & -2.79 & -2.77 & -2.75 & -2.73 \\
\hline & 30 & -3.25 & -3.15 & -3.07 & -3.03 & -3.02 & -2.97 & -3.04 & -2.98 & -2.93 & -2.90 & -2.88 & -2.86 & -2.93 & -2.89 & -2.84 & -2.82 & -2.81 & -2.79 \\
\hline 0 & 50 & -3.23 & -3.16 & -3.09 & -3.06 & -3.03 & -3.01 & -3.05 & -3.01 & -2.96 & -2.94 & -2.92 & -2.90 & -2.97 & -2.93 & -2.89 & -2.87 & -2.86 & -2.84 \\
\hline & 70 & -3.25 & -3.17 & -3.10 & -3.06 & -3.04 & -3.01 & -3.08 & -3.03 & -2.98 & -2.95 & -2.93 & -2.92 & -2.99 & -2.94 & -2.91 & -2.89 & -2.88 & -2.86 \\
\hline & 100 & -3.25 & -3.17 & -3.11 & -3.08 & -3.06 & -3.02 & -3.09 & -3.04 & -2.99 & -2.96 & -2.95 & -2.93 & -3.00 & -2.96 & -2.92 & -2.90 & -2.89 & -2.88 \\
\hline & 200 & -3.25 & -3.18 & -3.12 & -3.08 & -3.06 & -3.03 & -3.09 & -3.04 & -3.00 & -2.97 & -2.96 & -2.94 & -3.01 & -2.97 & -2.94 & -2.91 & -2.91 & -2.89 \\
\hline & 20 & -3.18 & -3.06 & -2.97 & -2.89 & -2.87 & -2.84 & -2.88 & -2.78 & -2.71 & -2.68 & -2.66 & -2.64 & -2.71 & -2.64 & -2.58 & -2.57 & -2.55 & -2.52 \\
\hline & 30 & .16 & -3.08 & -3.00 & -2.95 & -2.93 & -2.89 & -2.92 & -2.87 & -2.82 & -2.78 & -2.77 & -2.75 & -2.81 & -2.76 & -2.72 & -2.69 & -2.68 & -2.67 \\
\hline 1 & 50 & -3.21 & -3.11 & -3.04 & -3.01 & -2.99 & -2.96 & -3.00 & -2.95 & -2.90 & -2.87 & -2.86 & -2.84 & -2.90 & -2.86 & -2.82 & -2.80 & -2.79 & -2.77 \\
\hline & 70 & .21 & -3.14 & -3.07 & -3.03 & -3.01 & -2.98 & -3.06 & -2.99 & -2.94 & -2.91 & -2.89 & -2.87 & -2.95 & -2.90 & -2.86 & -2.84 & -2.83 & -2.82 \\
\hline & 100 & -3.24 & -3.15 & -3.09 & -3.05 & -3.03 & -3.00 & -3.06 & -3.01 & -2.96 & -2.93 & -2.92 & -2.90 & -2.96 & -2.93 & -2.89 & -2.87 & -2.86 & -2.85 \\
\hline & 200 & -3.25 & -3.17 & -3.12 & -3.08 & -3.05 & -3.02 & \begin{tabular}{|l|} 
\\
\end{tabular} & -3.03 & -2.99 & -2.96 & -2.95 & -2.93 & -3.00 & -2.95 & -2.92 & -2.90 & -2.89 & -2.87 \\
\hline & 20 & -3.43 & -3.17 & -2.92 & -2.82 & -2.75 & -2.61 & \begin{tabular}{|l|} 
\\
\end{tabular} & -2.64 & -2.51 & -2.45 & -2.41 & -2.34 & -2.52 & -2.42 & -2.33 & -2.28 & -2.25 & -2.21 \\
\hline & 30 & .92 & -2.86 & -2.76 & -2.71 & $\begin{array}{l}-2.69 \\
\end{array}$ & $\begin{array}{l}-2.64 \\
\end{array}$ & -2.68 & -2.63 & -2.55 & -2.53 & -2.51 & -2.49 & -2.56 & -2.51 & -2.46 & -2.43 & $\begin{array}{l}-2.42 \\
\end{array}$ & -2.40 \\
\hline 2 & 50 & .09 & -3.00 & -2.93 & -2.90 & -2.87 & -2.84 & -2.88 & $\begin{array}{l}-2.82 \\
\end{array}$ & -2.77 & -2.75 & -2.73 & -2.71 & $\begin{array}{l}-2.77 \\
\end{array}$ & -2.72 & -2.68 & -2.67 & -2.65 & -2.64 \\
\hline & 70 & .14 & -3.06 & -3.00 & -2.95 & -2.93 & -2.90 & -2.96 & -2.90 & -2.85 & -2.82 & -2.81 & -2.79 & -2.85 & -2.81 & -2.77 & -2.75 & -2.74 & -2.72 \\
\hline & 100 & 18 & -3.10 & -3.04 & -3.00 & -2.98 & -2.95 & -3.01 & -2.95 & -2.90 & -2.88 & -2.86 & -2.84 & -2.91 & -2.87 & -2.83 & -2.81 & -2.80 & -2.78 \\
\hline & 200 & -3.23 & -3.14 & -3.08 & -3.04 & -3.02 & -2.99 & -3.05 & -3.00 & -2.96 & -2.93 & -2.92 & -2.90 & -2.96 & -2.93 & -2.89 & -2.87 & -2.86 & -2.85 \\
\hline & 20 & - & & - & & - & & & - & & - & & & - & & & & & \\
\hline & 30 & -2.81 & -2.70 & -2.59 & -2.54 & -2.50 & -2.48 & -2.53 & -2.45 & -2.37 & -2.34 & -2.31 & -2.30 & -2.38 & -2.32 & -2.26 & -2.24 & -2.21 & -2.20 \\
\hline 3 & 50 & 0 & -2.92 & -2.85 & -2.83 & -2.80 & -2.76 & 30 & -2.74 & -2.69 & -2.66 & -2 . & -2.6 & -2 & & -2 & -2.57 & -2.56 & -2.55 \\
\hline & 70 & .09 & -3.03 & -2.95 & -2.91 & -2.88 & -2.86 & -2.91 & -2.85 & -2.80 & -2.77 & -2.76 & -2.74 & -2.81 & 75 & -2 & -2.70 & -2.68 & -2.67 \\
\hline & 100 & .16 & -3.07 & -3.02 & -2.97 & -2.96 & -2.93 & -2.97 & -2.92 & -2.87 & -2.84 & -2.83 & -2.81 & -2.87 & -2 & -2.80 & -2.78 & -2.76 & -2.75 \\
\hline & 200 & -3.21 & -3.14 & -3.07 & -3.03 & -3.01 & -2.99 & -3.04 & $\begin{array}{l}-2.99 \\
\end{array}$ & -2.95 & -2.92 & -2.90 & -2.89 & -2.95 & -2.91 & -2.88 & -2.85 & -2.85 & -2.83 \\
\hline & 20 & & & & & & & & & & & & & & & & & & \\
\hline & 30 & -2.63 & -2.47 & -2.34 & -2.28 & -2.21 & -2.18 & 2.31 & -2.18 & -2.10 & -2.06 & -2.02 & -1.98 & -2 . & 04 & -1. & -1.94 & -1.92 & -1.89 \\
\hline 4 & 50 & .86 & -2.79 & -2.71 & -2.69 & -2.66 & -2.61 & -2.65 & -2.59 & -2.54 & -2.52 & -2.49 & -2.47 & -2.54 & -2.49 & -2 & -2.42 & -2.41 & -2.39 \\
\hline & 70 & -2.99 & -2.93 & -2.87 & -2.83 & -2.79 & -2.77 & -2.81 & -2.75 & -2.70 & -2.67 & -2.66 & -2.64 & -2.71 & -2.66 & -2. & -2.60 & -2.58 & -2.57 \\
\hline & 100 & -3.10 & -3.02 & -2.95 & -2.92 & -2.91 & -2.87 & -2.91 & -2.85 & -2.81 & -2.79 & -2.77 & -2.75 & -2.80 & -2.76 & -2 & -2.71 & -2.70 & -2.68 \\
\hline & 200 & -3.19 & -3.11 & -3.05 & -3.01 & -2.98 & -2.96 & -3.01 & -2.96 & -2.92 & -2.89 & -2.88 & -2.86 & -2.92 & -2.88 & -2.85 & -2.83 & -2.81 & -2.80 \\
\hline & & & & & & & & & $k \dashv$ & $-1=4$ & & & & & & & & & \\
\hline & & & & $1 \%(\bar{C}$ & $A D F$ & & & & & $5 \%(\bar{C}$ & $A D F$ & & & & & $10 \%$ & $A D F$ & & \\
\hline$p$ & $(\mathrm{~T}, \mathrm{~N})$ & 20 & 30 & 50 & 70 & 100 & 200 & 20 & 30 & 50 & 70 & 100 & 200 & 20 & 30 & 50 & 70 & 100 & 200 \\
\hline & 20 & -3.39 & -3.29 & -3.19 & -3.13 & -3.09 & -3.08 & -3.10 & -3.03 & -2.97 & -2.94 & -2.92 & -2.90 & -2.96 & & -2.87 & -2.84 & -2.82 & -2.80 \\
\hline & 30 & .38 & -3.26 & -3.20 & -3.16 & -3.14 & -3.10 & -3.16 & -3.09 & -3.04 & -3.01 & -3.00 & -2.96 & -3.05 & -2.99 & -2 . & -2.93 & -2.91 & -2.89 \\
\hline 0 & 50 & .39 & -3.31 & -3.25 & -3.20 & -3.18 & -3.15 & -3.20 & -3.15 & -3.11 & -3.07 & -3.06 & -3.04 & -3.11 & -3.07 & -3. & -3.01 & -2.99 & -2.98 \\
\hline & 70 & 41 & -3.32 & -3.25 & -3.24 & -3.19 & -3.17 & -3.23 & -3.17 & -3.13 & -3.10 & -3.09 & -3.07 & -3.13 & -3.09 & -3 . & -3.04 & -3.02 & -3.01 \\
\hline & 100 & .41 & -3.33 & -3.28 & -3.24 & -3.22 & -3.18 & -3.25 & -3.20 & -3.15 & -3.12 & -3.10 & -3.09 & -3.16 & -3.12 & -3. & -3.06 & -3.04 & -3.03 \\
\hline & 200 & -3.43 & -3.34 & -3.30 & -3.26 & -3.23 & -3.2 & -3.2 & -3.21 & -3.1 & -3 & -3.13 & -3.11 & -3.18 & -3 & -3 . & -3.09 & -3.07 & -3.05 \\
\hline & 20 & -3.43 & -3.26 & -3.05 & -2.99 & -2.93 & -2.86 & -2.97 & -2.85 & -2.74 & -2.68 & -2.65 & -2.61 & -2.74 & -2.66 & -2.59 & -2.53 & -2.51 & -2.48 \\
\hline & 30 & .23 & -3.13 & -3.03 & -3.00 & -2.97 & -2.93 & -2.97 & -2.90 & -2.84 & -2.81 & -2.79 & -2.77 & -2.83 & & -2 . & -2.72 & -2.70 & -2.68 \\
\hline 1 & 50 & -3.31 & -3.22 & -3.17 & -3.12 & -3.09 & -3.07 & -3.11 & -3.05 & -3.00 & -2.98 & -2.96 & -2.94 & -3.00 & -2.96 & -2.92 & -2.90 & -2.89 & -2.87 \\
\hline & 70 & & -3.26 & -3.21 & -3.18 & -3.14 & -3.1 & -3 & -3.11 & -3.0 & -3. & -3. & -3.1 & -3 & & -2 & -2 & & -2.94 \\
\hline & 100 & -3.37 & -3.29 & -3.23 & -3.20 & -3.18 & -3.15 & -3.20 & -3.15 & -3.10 & -3.08 & -3.05 & -3.04 & -3.11 & -3.07 & -3.03 & -3.01 & -2.99 & -2.98 \\
\hline & 200 & -3.42 & -3.34 & -3.27 & -3.24 & -3.22 & -3.19 & -3.25 & -3.19 & -3.15 & -3.13 & -3.10 & -3.08 & -3.16 & -3.11 & -3.08 & -3.06 & -3.05 & -3.03 \\
\hline & 20 & & & & & - & & & & & & & & & & & & & \\
\hline & 30 & & & -2.67 & -2.62 & & -2.5 & & & & & -2.41 & & & & & & & -2.29 \\
\hline 2 & 50 & .17 & -3.06 & -2.99 & -2.96 & -2.92 & -2.9 & -2. & -2.87 & -2.8 & -2.80 & -2.78 & -2 . & -2 & -2 & -2 . & -2.71 & -2.70 & -2.68 \\
\hline & 70 & & -3. & -3.09 & -3.1 & -3. & -3. & -3. & -2 . & -2 & -2 . & -2 . & & & & & & & -2.81 \\
\hline & 100 & -3 & -3.23 & -3.16 & -3.13 & -3 . & -3. & -3 . & -3 . & -3 . & -3. & -2 . & -2 . & & -2 & -2 . & -2 & -2.91 & -2.90 \\
\hline & 200 & -3.37 & -3.29 & -3.24 & -3.20 & -3.18 & -3.15 & $\mid-3.21$ & -3.15 & -3.11 & -3.09 & -3.07 & -3.05 & -3.12 & -3.07 & -3.04 & -3.03 & -3.01 & -2.99 \\
\hline & 20 & - & & & & & - & & & & & & & & & & & & \\
\hline & 30 & -2.85 & -2.75 & -2.53 & -2.45 & -2.36 & -2.3 & -2 . & -2.36 & -2 & -2 . & -2.14 & -2.1 & & & -2 . & & & -1.99 \\
\hline 3 & 50 & .04 & -2.94 & -2.86 & -2.83 & -2.79 & -2.7 & -2 & -2.74 & -2 & -2. & -2.64 & -2.6 & -2. & & -2 & -2.57 & -2.55 & -2.53 \\
\hline & 70 & & -3.11 & -3.02 & -2.9 & -2.9 & -2. & -2 & -2.9 & -2. & -2.8 & -2.81 & -2 . & -2.8 & & -2 . & & & -2.72 \\
\hline & 100 & & -3.19 & -3.12 & -3.0 & -3.06 & -3.0 & -3. & -3.0 & -2. & -2.9 & -2.93 & -2 & -2. & & -2 . & -2.87 & -2.86 & -2.85 \\
\hline & 200 & -3.34 & -3.28 & -3.22 & -3.19 & -3.16 & -3.13 & -3.18 & -3.13 & -3.09 & -3.06 & -3.05 & -3.03 & -3.09 & -3.05 & -3.02 & -3.00 & -2.98 & -2.97 \\
\hline & 20 & & & & & & & & & & & & & & & & & & - \\
\hline & 30 & & - & - & & - & & & - & - & - & - & - & - & & - & - & - & - \\
\hline 4 & 50 & -2.83 & -2.72 & -2.66 & -2.62 & -2.58 & -2.54 & -2.59 & -2.53 & -2.47 & -2.44 & -2.42 & -2.38 & -2.47 & -2.42 & -2.37 & -2.35 & -2.33 & -2.30 \\
\hline & 70 & & -2.97 & -2.89 & -2.85 & -2.82 & -2.80 & -2.84 & -2.78 & -2.7 & -2. & -2.68 & -2.6 & -2 & -2.67 & -2. & -2.61 & -2.60 & -2.58 \\
\hline & 100 & & -3.11 & -3.05 & -3.00 & -2.99 & -2.95 & -2.98 & -2.94 & -2.89 & -2.86 & -2.84 & -2.83 & -2.88 & -2.85 & -2.81 & -2.78 & -2.77 & -2.76 \\
\hline & 200 & -3.32 & -3.24 & -3.18 & -3.15 & -3.12 & -3.10 & -3.15 & -3.09 & -3.05 & -3.03 & -3.01 & -2.99 & -3.05 & -3.01 & -2.98 & -2.96 & -2.94 & -2.93 \\
\hline
\end{tabular}

Notes: The critical values are obtained by stochastic simulation. The data generating process is $y_{i t}=y_{i t-1}+f_{t}+u_{i t}$ with $f_{t} \sim \operatorname{iidN}(0,1), u_{i t} \sim \operatorname{iidN}(0,1)$, with $y_{i 0}=0$, and the $j^{\text {th }}$ element of the $k \times 1$ vector of additional regressors, $x_{i t}$, are generated as $x_{i j t}=x_{i j t-1}+f_{j t}+v_{i j t}$, with $f_{j t} \sim i i d N(0,1), v_{i j t} \sim i i d N(0,1)$ and $x_{i j 0}=0, i=1,2, \ldots, N ; j=1,2, \ldots, k$; $t=-p, \ldots, T$. Based on these data, $C A D F_{i}$, which is the $t$-ratio of coefficient on $y_{i t-1}$ of regression of $\Delta y_{i t}$ on $y_{i t-1}$, 
$w_{i t, p}^{\prime}=\left(\bar{z}_{t-1}^{\prime} ; \Delta \bar{z}_{t}^{\prime}, \Delta \bar{z}_{t-1}^{\prime}, \ldots, \Delta \bar{z}_{t-p}^{\prime} ; \Delta y_{i, t-1}, \ldots, \Delta y_{i, t-p}\right)$ with $\bar{z}_{t}=N^{-1} \sum_{i=1}^{N}\left(y_{i t}, x_{i t}^{\prime}\right)^{\prime}$ and deterministics as specified, then $\overline{C A D F}=N^{-1} \sum_{i=1}^{N} C A D F_{i}$ are computed. $(100 \times \alpha) \%$ critical values are obtained as the $1-\alpha$ quantiles of $\overline{C A D F}$ for $\alpha=0.01,0.05,0.1$. Computations are based on 10000 replications. 
Table 2: Size and Power of Panel Unit Root Tests with Two Factors $(m=2)$, Serially Correlated $\Delta v_{i x t}$, Intercept Only Case

\begin{tabular}{|c|c|c|c|c|c|c|c|c|c|c|c|c|}
\hline & \multicolumn{6}{|c|}{ Size: $\rho_{i}=\rho=1$} & \multicolumn{6}{|c|}{ Power: $\rho_{i} \sim$ iidU $[0.90,0.99]$} \\
\hline$(\mathrm{T}, \mathrm{N})$ & 20 & 30 & 50 & 70 & 100 & 200 & 20 & 30 & 50 & 70 & 100 & 200 \\
\hline $\operatorname{IPS}(p)$ & \multicolumn{6}{|c|}{$p=0$} & \multicolumn{6}{|c|}{$p=0$} \\
\hline 20 & 31.85 & 33.35 & 39.90 & 41.65 & 42.65 & 44.95 & 37.65 & 38.75 & 46.55 & 49.40 & 49.15 & 55.50 \\
\hline 30 & 31.70 & 34.30 & 40.50 & 41.60 & 44.95 & 47.65 & 39.35 & 47.50 & 51.65 & 54.00 & 57.75 & 59.00 \\
\hline 50 & 34.10 & 36.95 & 39.95 & 42.65 & 45.95 & 48.40 & 51.10 & 56.60 & 59.20 & 64.35 & 65.90 & 69.05 \\
\hline 70 & 35.30 & 36.50 & 42.10 & 41.25 & 46.65 & 46.20 & 59.20 & 67.75 & 70.75 & 73.45 & 74.70 & 76.50 \\
\hline 100 & 32.90 & 35.85 & 41.05 & 41.80 & 45.40 & 47.55 & 71.75 & 78.20 & 82.60 & 84.55 & 85.60 & 87.45 \\
\hline 200 & 32.30 & 35.70 & 40.35 & 42.35 & 44.50 & 47.60 & 94.25 & 97.50 & 98.15 & 98.75 & 98.90 & 99.25 \\
\hline $\operatorname{CIPS}(p)$ & \multicolumn{6}{|c|}{$p=0$} & \multicolumn{6}{|c|}{$p=0$} \\
\hline 20 & 4.75 & 4.85 & 4.35 & 4.95 & 5.00 & 5.55 & 4.15 & 5.00 & 4.70 & 4.85 & 4.95 & 5.55 \\
\hline 30 & 5.00 & 4.85 & 4.75 & 4.50 & 5.15 & 5.10 & 5.95 & 7.05 & 6.90 & 6.35 & 6.15 & 6.35 \\
\hline 50 & 5.05 & 4.10 & 4.70 & 5.75 & 4.90 & 4.30 & 11.70 & 15.70 & 18.70 & 19.75 & 16.25 & 24.60 \\
\hline 70 & 4.65 & 4.85 & 5.10 & 5.25 & 4.05 & 4.40 & 20.90 & 37.70 & 44.35 & 47.80 & 47.60 & 65.65 \\
\hline 100 & 4.45 & 4.95 & 4.15 & 4.85 & 5.75 & 4.85 & 48.00 & 77.05 & 87.40 & 91.80 & 94.60 & 99.40 \\
\hline 200 & 4.55 & 5.25 & 5.35 & 4.70 & 5.30 & 4.55 & 99.90 & 100.00 & 100.00 & 100.00 & 100.00 & 100.00 \\
\hline \multicolumn{13}{|l|}{$P_{b}$} \\
\hline 20 & 9.05 & 9.45 & 9.20 & 11.55 & 14.15 & 20.30 & 17.75 & 29.00 & 37.65 & 44.80 & 52.75 & 72.90 \\
\hline 30 & 6.85 & 7.30 & 8.90 & 8.60 & 9.60 & 14.00 & 28.70 & 50.35 & 61.90 & 69.20 & 73.25 & 80.05 \\
\hline 50 & 7.70 & 7.90 & 8.00 & 7.05 & 7.70 & 8.45 & 51.50 & 81.25 & 84.60 & 83.30 & 85.60 & 89.55 \\
\hline 70 & 7.65 & 6.45 & 7.10 & 6.20 & 6.45 & 7.90 & 70.75 & 93.55 & 92.00 & 90.85 & 93.30 & 93.55 \\
\hline 100 & 6.50 & 7.95 & 7.50 & 6.05 & 6.55 & 5.90 & 82.45 & 98.30 & 97.10 & 95.10 & 96.25 & 97.70 \\
\hline 200 & 8.05 & 6.05 & 6.35 & 6.35 & 4.65 & 5.10 & 96.30 & 100.00 & 99.80 & 99.30 & 99.65 & 99.85 \\
\hline \multicolumn{13}{|l|}{$P M S B$} \\
\hline 20 & 2.20 & 2.85 & 1.85 & 2.80 & 3.70 & 5.30 & 4.20 & 7.85 & 6.90 & 9.25 & 12.45 & 24.55 \\
\hline 30 & 3.00 & 2.65 & 2.55 & 2.50 & 2.70 & 4.25 & 10.00 & 20.80 & 28.35 & 34.95 & 43.10 & 61.15 \\
\hline 50 & 4.50 & 4.20 & 3.60 & 2.80 & 3.05 & 2.70 & 30.25 & 60.70 & 68.85 & 71.90 & 74.95 & 82.60 \\
\hline 70 & 4.50 & 4.30 & 4.50 & 3.15 & 3.15 & 3.05 & 50.80 & 84.20 & 85.05 & 85.25 & 86.95 & 88.40 \\
\hline 100 & 5.00 & 5.50 & 4.50 & 3.35 & 3.85 & 3.60 & 69.55 & 95.00 & 93.80 & 91.95 & 93.00 & 95.75 \\
\hline 200 & 6.50 & 4.55 & 4.80 & 5.40 & 3.40 & 3.35 & 92.85 & 100.00 & 99.40 & 98.75 & 99.40 & 99.25 \\
\hline \multicolumn{13}{|l|}{$t_{b}^{*}$} \\
\hline 20 & 15.80 & 13.80 & 19.15 & 21.00 & 25.65 & 31.05 & 69.75 & 87.70 & 89.40 & 89.10 & 89.30 & 93.00 \\
\hline 30 & 13.05 & 12.20 & 15.80 & 16.20 & 19.15 & 28.00 & 78.00 & 94.90 & 94.35 & 93.60 & 93.10 & 95.75 \\
\hline 50 & 9.70 & 8.35 & 12.00 & 13.00 & 13.50 & 21.30 & 86.40 & 98.75 & 98.10 & 96.15 & 97.60 & 98.00 \\
\hline 70 & 9.05 & 7.30 & 10.15 & 10.75 & 12.65 & 17.25 & 93.00 & 99.85 & 99.20 & 98.30 & 99.30 & 99.45 \\
\hline 100 & 9.75 & 7.70 & 10.25 & 8.85 & 10.60 & 13.35 & 96.35 & 100.00 & 99.75 & 99.75 & 99.60 & 99.80 \\
\hline 200 & 9.70 & 7.05 & 7.65 & 6.80 & 7.40 & 8.70 & 99.70 & 100.00 & 100.00 & 100.00 & 100.00 & 100.00 \\
\hline
\end{tabular}

Notes: $y_{i t}$ is generated as $y_{i t}=\left(1-\rho_{i}\right) \alpha_{i}+\rho_{i} y_{i, t-1}+\gamma_{i 1} f_{1 t}+\gamma_{i 2} f_{2 t}+\varepsilon_{i t}, i=1,2, \ldots, N ; t=-49, \ldots, T$ with $y_{i,-50}=0$, where $\rho_{i}=\rho=1$ for size and $\rho_{i} \sim \operatorname{iidU}[0.90,0.99]$ for power; $\alpha_{i} \sim \operatorname{iidN}(1,1) ; f_{j t}=\rho_{f j} f_{f j, t-1}+\varpi_{j t}, \varpi_{j t} \sim \operatorname{iidN}(0,1)$ with $f_{j,-50}=0$ and $\rho_{f j}=0$ for $j=1,2 ; \varepsilon_{i t}=\rho_{i \varepsilon} \varepsilon_{i t-1}+\zeta_{i t}, \zeta_{i t} \sim \operatorname{iidN}\left(0, \sigma_{i}^{2}\right)$ with $\varepsilon_{i,-50}=0$ and $\rho_{i \varepsilon}=\rho_{\varepsilon}=0$, $\sigma_{i}^{2} \sim i i d U[0.5,1.5] . x_{i j t}$ is generated as $x_{i j t}=\mu_{i j}+\gamma_{i j x 1} f_{1 t}+\gamma_{i j x 2} f_{2 t}+v_{i j x t}, i=1,2, \ldots, N ; j=1,2 ; t=-49, \ldots, T$ with $x_{i j,-50}=0$, where $\mu_{i j} \sim \operatorname{iidN}(1,1) ; v_{i j x t}=v_{i j x t-1}+e_{i j v x t}, \quad e_{i j v x t}=\rho_{i j v x} e_{i j v x, t-1}+\varrho_{i j t}, \quad \varrho_{i j t} \sim \operatorname{iidN}(0,1)$ with $v_{i j x,-50}=0, e_{i j v x,-50}=0$, and $\rho_{i j v x} \sim \operatorname{iid} U[0.2,0.4]$. The factor loadings are generated as $\gamma_{i 1} \sim \operatorname{iidU}[1,3]$, $\gamma_{i 2} \sim i i d U[0,2], \gamma_{i j x 1} \sim \operatorname{iidU}[0,2], \gamma_{i j x 2} \sim \operatorname{iidU}[1,3]$, so that the rank condition (9) is satisfied. $\alpha_{i}, \gamma_{i 1}, \gamma_{i 2}, \rho_{f j}, \rho_{i}, \mu_{i j}$, $\gamma_{i j x 1}, \gamma_{i j x 2}$ and $\rho_{i j v x}$ are drawn once and fixed over all replications. The IPS(p) test is the Im et al. (2003) panel unit root test with lag-augmentation of order $p$. The CIPS(p) test is the proposed panel unit root test, defined by (35), based on cross section augmentation using $y_{i t}, x_{i 1 t}$ and,$x_{i 2 t}(k=2)$ with lag-augmentation of order $p$. The $P_{b}$ and $P M S B$ tests are the Bai and $\mathrm{Ng}$ (2007) pooled panel unit root tests for the idiosyncratic errors. The $t_{b}^{*}$ test is the Moon and Perron (2004) panel unit root test for the idiosyncratic errors. The $P_{b}, P M S B$, and $t_{b}^{*}$ tests are based on two extracted factors from the $y_{i t}$ series using principal components, and they adopt automatic lag-order selection for the estimation of long-run variances following Andrews and Monahan (1992). All tests are conducted at the 5\% significance level, and the CIPS(p) test is based on critical values for different $p$ and $k$. All experiments are based on 2000 replications. 
Table 3: Size and Power of Panel Unit Root Tests with Two Factors $(m=2)$, Positively Serially Correlated $\varepsilon_{i t}$ and $\Delta v_{i x t}$, Intercept Only Case

\begin{tabular}{|c|c|c|c|c|c|c|c|c|c|c|c|c|}
\hline & \multicolumn{6}{|c|}{ Size: $\rho_{i}=\rho=1$} & \multicolumn{6}{|c|}{ Power: $\rho_{i} \sim i i d U[0.90,0.99]$} \\
\hline$(\mathrm{T}, \mathrm{N})$ & 20 & 30 & 50 & 70 & 100 & 200 & 20 & 30 & 50 & 70 & 100 & 200 \\
\hline $\operatorname{IPS}(p)$ & \multicolumn{6}{|c|}{$p=1$} & \multicolumn{6}{|c|}{$p=1$} \\
\hline 20 & 27.50 & 29.40 & 35.10 & 38.00 & 38.60 & 42.85 & 33.80 & 37.20 & 43.70 & 47.15 & 48.10 & 52.60 \\
\hline 30 & 29.30 & 29.20 & 35.95 & 37.35 & 43.10 & 45.80 & 38.70 & 43.35 & 51.10 & 53.35 & 56.50 & 62.10 \\
\hline 50 & 27.35 & 30.15 & 35.35 & 36.95 & 43.55 & 42.95 & 49.15 & 54.70 & 61.50 & 63.10 & 66.10 & 69.75 \\
\hline 70 & 28.60 & 30.05 & 33.90 & 38.45 & 42.60 & 46.50 & 57.65 & 65.50 & 68.55 & 73.30 & 75.80 & 78.75 \\
\hline 100 & 26.90 & 30.00 & 38.45 & 38.85 & 40.25 & 45.95 & 67.95 & 77.90 & 81.05 & 83.65 & 84.70 & 87.65 \\
\hline 200 & 28.85 & 29.85 & 36.90 & 38.40 & 40.25 & 44.90 & 95.35 & 97.40 & 98.20 & 98.70 & 99.00 & 99.00 \\
\hline $\operatorname{CIPS}(p)$ & \multicolumn{6}{|c|}{$p=1$} & \multicolumn{6}{|c|}{$p=1$} \\
\hline 20 & 4.50 & 3.85 & 3.50 & 2.65 & 3.40 & 2.60 & 4.50 & 4.50 & 5.15 & 4.20 & 4.05 & 5.05 \\
\hline 30 & 5.35 & 3.40 & 3.40 & 2.65 & 3.75 & 2.90 & 6.65 & 5.55 & 6.25 & 6.60 & 5.90 & 7.05 \\
\hline 50 & 4.10 & 4.05 & 3.70 & 3.55 & 3.40 & 3.80 & 9.30 & 12.60 & 16.35 & 15.55 & 13.70 & 19.30 \\
\hline 70 & 4.75 & 4.95 & 4.65 & 4.30 & 4.35 & 4.00 & 18.95 & 27.15 & 34.15 & 34.40 & 33.55 & 46.95 \\
\hline 100 & 4.70 & 5.25 & 3.85 & 4.70 & 4.70 & 5.15 & 40.50 & 61.45 & 73.80 & 77.45 & 81.90 & 92.60 \\
\hline 200 & 5.10 & 5.25 & 4.60 & 3.90 & 4.35 & 4.60 & 98.70 & 100.00 & 100.00 & 100.00 & 100.00 & 100.00 \\
\hline \multicolumn{13}{|l|}{$P_{b}$} \\
\hline 20 & 7.05 & 7.20 & 9.45 & 13.35 & 14.45 & 23.00 & 17.15 & 24.90 & 37.95 & 46.15 & 57.95 & 79.15 \\
\hline 30 & 6.75 & 5.50 & 7.15 & 8.15 & 10.75 & 15.05 & 31.10 & 50.20 & 67.95 & 74.70 & 82.25 & 89.70 \\
\hline 50 & 6.80 & 6.40 & 5.90 & 5.75 & 7.60 & 9.15 & 58.70 & 86.75 & 91.55 & 92.55 & 94.50 & 96.90 \\
\hline 70 & 7.35 & 7.15 & 6.00 & 5.60 & 6.70 & 7.80 & 78.50 & 97.25 & 97.55 & 97.75 & 97.85 & 98.65 \\
\hline 100 & 6.25 & 6.50 & 5.75 & 5.80 & 6.10 & 7.10 & 92.25 & 99.85 & 98.70 & 99.15 & 99.25 & 99.65 \\
\hline 200 & 7.30 & 6.50 & 5.55 & 5.30 & 5.00 & 6.25 & 99.25 & 100.00 & 99.95 & 100.00 & 100.00 & 100.00 \\
\hline \multicolumn{13}{|l|}{$P M S B$} \\
\hline 20 & 0.10 & 0.10 & 0.00 & 0.05 & 0.25 & 0.60 & 0.05 & 0.10 & 0.05 & 0.05 & 0.05 & 0.50 \\
\hline 30 & 0.00 & 0.00 & 0.00 & 0.00 & 0.05 & 0.20 & 0.10 & 0.50 & 0.30 & 0.55 & 1.05 & 1.20 \\
\hline 50 & 0.10 & 0.00 & 0.00 & 0.00 & 0.00 & 0.00 & 0.75 & 4.50 & 8.20 & 12.00 & 24.05 & 48.80 \\
\hline 70 & 0.05 & 0.00 & 0.00 & 0.00 & 0.00 & 0.00 & 4.40 & 20.45 & 38.50 & 46.25 & 65.60 & 80.15 \\
\hline 100 & 0.00 & 0.00 & 0.00 & 0.00 & 0.00 & 0.00 & 16.95 & 59.95 & 76.70 & 82.50 & 88.25 & 91.00 \\
\hline 200 & 0.00 & 0.00 & 0.00 & 0.00 & 0.00 & 0.00 & 78.45 & 99.25 & 99.05 & 99.35 & 99.50 & 99.70 \\
\hline \multicolumn{13}{|l|}{$t_{b}^{*}$} \\
\hline 20 & 12.70 & 11.10 & 15.65 & 14.10 & 20.15 & 25.40 & 75.75 & 92.20 & 93.75 & 94.25 & 96.35 & 97.80 \\
\hline 30 & 9.30 & 8.25 & 12.60 & 11.70 & 15.00 & 21.55 & 85.70 & 97.75 & 98.05 & 97.15 & 98.05 & 99.00 \\
\hline 50 & 7.90 & 6.40 & 8.85 & 9.15 & 11.20 & 16.15 & 93.10 & 99.75 & 99.65 & 99.30 & 99.65 & 99.85 \\
\hline 70 & 8.00 & 6.80 & 8.30 & 8.30 & 10.30 & 13.35 & 97.65 & 100.00 & 99.90 & 99.90 & 99.75 & 99.90 \\
\hline 100 & 7.60 & 6.60 & 8.20 & 7.65 & 6.95 & 10.65 & 99.30 & 100.00 & 99.95 & 99.95 & 100.00 & 100.00 \\
\hline 200 & 7.60 & 7.05 & 6.60 & 7.05 & 6.10 & 7.65 & 100.00 & 100.00 & 100.00 & 100.00 & 100.00 & 100.00 \\
\hline
\end{tabular}

Notes: See notes to Table 2. The data generating process is the same as the one for Table 2, except $\rho_{i \varepsilon} \sim i i d U[0.2,0.4]$. 
Table 4: Size and Power of Panel Unit Root Tests with Two Factors $(m=2)$, Negatively Serially Correlated $\varepsilon_{i t}$ and Positively Serially Correlated $\Delta v_{i x t}$, Intercept Only Case

\begin{tabular}{|c|c|c|c|c|c|c|c|c|c|c|c|c|}
\hline & \multicolumn{6}{|c|}{ Size: $\rho_{i}=\rho=1$} & \multicolumn{6}{|c|}{ Power: $\rho_{i} \sim \operatorname{iidU[0.90,0.99]}$} \\
\hline$(\mathrm{T}, \mathrm{N})$ & 20 & 30 & 50 & 70 & 100 & 200 & 20 & 30 & 50 & 70 & 100 & 200 \\
\hline $\operatorname{IPS}(p)$ & \multicolumn{6}{|c|}{$p=1$} & \multicolumn{6}{|c|}{$p=1$} \\
\hline 20 & 31.20 & 34.85 & 40.10 & 42.30 & 40.60 & 45.75 & 37.20 & 40.50 & 45.30 & 49.20 & 49.85 & 52.40 \\
\hline 30 & 33.65 & 35.40 & 39.70 & 40.95 & 45.75 & 47.95 & 41.00 & 45.30 & 51.90 & 52.80 & 55.50 & 60.00 \\
\hline 50 & 30.75 & 36.05 & 39.80 & 41.40 & 46.20 & 44.20 & 48.60 & 53.80 & 59.60 & 61.15 & 63.25 & 65.75 \\
\hline 70 & 32.85 & 35.85 & 37.60 & 41.85 & 44.75 & 48.55 & 56.40 & 62.40 & 65.70 & 70.00 & 72.55 & 74.40 \\
\hline 100 & 31.35 & 35.00 & 41.65 & 42.80 & 42.25 & 47.05 & 65.15 & 74.50 & 76.75 & 79.40 & 80.85 & 84.15 \\
\hline 200 & 34.20 & 35.60 & 40.80 & 42.60 & 42.50 & 46.45 & 92.60 & 95.40 & 96.80 & 97.25 & 98.15 & 98.05 \\
\hline $\operatorname{CIPS}(p)$ & \multicolumn{6}{|c|}{$p=1$} & \multicolumn{6}{|c|}{$p=1$} \\
\hline 20 & 6.25 & 6.45 & 6.40 & 6.15 & 6.35 & 6.75 & 4.40 & 4.65 & 5.65 & 4.70 & 4.10 & 3.65 \\
\hline 30 & 6.25 & 4.55 & 5.25 & 5.70 & 5.70 & 5.50 & 4.50 & 4.15 & 4.40 & 4.25 & 3.65 & 4.30 \\
\hline 50 & 5.00 & 5.00 & 5.80 & 5.35 & 5.20 & 5.75 & 7.20 & 11.30 & 13.05 & 10.75 & 8.70 & 13.00 \\
\hline 70 & 4.85 & 5.35 & 5.50 & 5.30 & 5.30 & 5.40 & 18.65 & 31.75 & 38.15 & 39.65 & 35.20 & 58.90 \\
\hline 100 & 4.20 & 5.75 & 4.55 & 4.95 & 5.85 & 6.10 & 48.60 & 77.95 & 87.95 & 92.00 & 93.55 & 99.15 \\
\hline 200 & 4.70 & 5.35 & 5.30 & 4.05 & 4.60 & 5.00 & 99.95 & 100.00 & 100.00 & 100.00 & 100.00 & 100.00 \\
\hline \multicolumn{13}{|l|}{$P_{b}$} \\
\hline 20 & 11.70 & 10.80 & 13.40 & 16.15 & 17.50 & 28.90 & 22.60 & 36.15 & 43.85 & 48.85 & 54.30 & 67.50 \\
\hline 30 & 10.50 & 9.05 & 10.60 & 10.70 & 12.65 & 18.05 & 31.10 & 51.35 & 57.80 & 61.45 & 65.20 & 69.25 \\
\hline 50 & 8.40 & 9.35 & 8.55 & 8.25 & 10.55 & 12.30 & 45.15 & 77.30 & 72.65 & 73.00 & 75.70 & 77.30 \\
\hline 70 & 9.00 & 9.40 & 7.70 & 7.90 & 8.45 & 10.70 & 58.70 & 87.45 & 81.45 & 79.15 & 82.00 & 83.55 \\
\hline 100 & 7.90 & 8.30 & 8.35 & 7.20 & 8.25 & 8.70 & 71.15 & 94.35 & 88.40 & 88.30 & 87.30 & 87.80 \\
\hline 200 & 8.10 & 7.15 & 6.45 & 6.05 & 6.35 & 7.30 & 90.30 & 99.50 & 98.00 & 97.35 & 97.35 & 97.85 \\
\hline \multicolumn{13}{|l|}{$P M S B$} \\
\hline 20 & 25.80 & 28.00 & 37.10 & 45.20 & 52.20 & 77.20 & 31.85 & 50.90 & 57.55 & 59.70 & 63.75 & 74.85 \\
\hline 30 & 32.50 & 35.40 & 50.80 & 59.00 & 69.45 & 91.05 & 46.90 & 72.15 & 72.90 & 74.35 & 75.25 & 78.35 \\
\hline 50 & 38.85 & 48.45 & 61.20 & 71.80 & 80.40 & 97.20 & 64.65 & 91.05 & 83.10 & 83.85 & 83.10 & 84.80 \\
\hline 70 & 43.75 & 52.80 & 66.95 & 75.40 & 84.70 & 97.85 & 74.75 & 95.40 & 89.65 & 87.85 & 87.90 & 89.75 \\
\hline 100 & 48.40 & 54.65 & 69.00 & 80.05 & 86.25 & 99.05 & 83.35 & 98.30 & 93.55 & 92.50 & 92.90 & 92.25 \\
\hline 200 & 49.45 & 59.20 & 74.25 & 82.30 & 89.60 & 98.85 & 95.05 & 100.00 & 99.25 & 99.00 & 99.10 & 98.95 \\
\hline \multicolumn{13}{|l|}{$t_{b}^{*}$} \\
\hline 20 & 17.60 & 16.90 & 22.55 & 22.50 & 30.45 & 37.45 & 65.45 & 83.90 & 83.00 & 81.45 & 84.60 & 86.95 \\
\hline 30 & 13.60 & 13.95 & 19.90 & 19.15 & 24.65 & 34.10 & 69.60 & 90.65 & 87.75 & 85.60 & 88.00 & 89.00 \\
\hline 50 & 11.15 & 10.50 & 15.40 & 15.60 & 18.85 & 26.40 & 78.95 & 96.05 & 92.75 & 90.90 & 93.45 & 93.40 \\
\hline 70 & 10.10 & 9.15 & 11.80 & 12.70 & 17.05 & 20.85 & 85.10 & 98.25 & 96.25 & 95.25 & 94.75 & 95.60 \\
\hline 100 & 9.50 & 9.05 & 11.25 & 10.70 & 11.75 & 18.20 & 90.95 & 99.55 & 98.25 & 97.40 & 97.55 & 98.15 \\
\hline 200 & 8.45 & 8.10 & 7.20 & 8.30 & 8.60 & 10.40 & 98.45 & 100.00 & 99.95 & 99.90 & 99.90 & 99.75 \\
\hline
\end{tabular}

Notes: See notes to Table 2. The data generating process is the same as the one for Table 2, except $\rho_{i \varepsilon} \sim i i d U[-0.2,-0.4]$. 
Table 5: Size of Panel Unit Root Tests with Two Factors $(m=2)$, Serially Correlated $f_{t}$ and $\Delta v_{i x t}$, Intercept Only Case

\begin{tabular}{|c|c|c|c|c|c|c|c|c|c|c|c|c|}
\hline$(\mathrm{T}, \mathrm{N})$ & 20 & 30 & 50 & 70 & 100 & 200 & 20 & 30 & 50 & 70 & 100 & 200 \\
\hline $\operatorname{IPS}(p)$ & \multicolumn{6}{|c|}{$p=0$} & \multicolumn{6}{|c|}{$p=1$} \\
\hline 20 & 17.85 & 18.75 & 22.15 & 24.40 & 25.90 & 28.95 & 31.30 & 34.60 & 39.40 & 42.25 & 41.15 & 44.90 \\
\hline 30 & 18.25 & 17.90 & 22.20 & 24.10 & 26.60 & 29.35 & 33.10 & 34.90 & 40.40 & 40.75 & 46.15 & 47.90 \\
\hline 50 & 15.35 & 18.95 & 22.20 & 19.75 & 24.95 & 25.45 & 30.50 & 35.90 & 39.45 & 40.40 & 46.10 & 44.10 \\
\hline 70 & 16.70 & 17.05 & 20.55 & 21.85 & 24.80 & 27.55 & 33.00 & 34.90 & 37.75 & 41.30 & 44.30 & 47.75 \\
\hline 100 & 14.70 & 16.45 & 23.35 & 22.55 & 22.95 & 27.40 & 31.35 & 34.80 & 41.60 & 42.40 & 41.85 & 47.25 \\
\hline 200 & 16.50 & 17.40 & 21.60 & 21.70 & 23.75 & 26.20 & 33.95 & 34.95 & 40.80 & 42.35 & 42.10 & 45.60 \\
\hline $\operatorname{CIPS}(p)$ & \multicolumn{6}{|c|}{$p=0$} & \multicolumn{6}{|c|}{$p=1$} \\
\hline 20 & 12.70 & 13.75 & 14.35 & 16.95 & 17.75 & 20.10 & 10.35 & 11.30 & 10.60 & 11.05 & 12.05 & 13.80 \\
\hline 30 & 10.60 & 9.20 & 11.20 & 11.55 & 13.40 & 14.00 & 8.90 & 7.90 & 8.95 & 9.95 & 10.15 & 10.40 \\
\hline 50 & 8.25 & 8.10 & 9.85 & 9.75 & 10.50 & 11.45 & 7.10 & 6.65 & 8.25 & 8.00 & 8.05 & 8.45 \\
\hline 70 & 7.25 & 7.70 & 8.35 & 8.60 & 9.75 & 8.35 & 6.60 & 7.10 & 7.75 & 6.60 & 8.00 & 6.40 \\
\hline 100 & 5.20 & 7.30 & 6.35 & 7.50 & 8.15 & 8.60 & 5.55 & 6.75 & 5.35 & 6.10 & 7.40 & 7.80 \\
\hline 200 & 5.50 & 5.90 & 6.65 & 4.75 & 5.45 & 6.05 & 5.60 & 6.00 & 5.80 & 4.95 & 5.15 & 5.65 \\
\hline \multicolumn{13}{|l|}{$P_{b}$} \\
\hline 20 & - & - & - & - & - & - & 11.65 & 10.45 & 14.45 & 18.50 & 20.55 & 30.30 \\
\hline 30 & - & - & - & - & - & - & 8.75 & 7.35 & 10.30 & 11.25 & 15.35 & 20.40 \\
\hline 50 & - & - & - & - & - & - & 7.65 & 7.80 & 8.20 & 7.15 & 10.30 & 12.75 \\
\hline 70 & - & - & - & - & - & - & 8.35 & 8.60 & 7.05 & 7.20 & 8.50 & 10.35 \\
\hline 100 & - & - & - & - & - & - & 7.10 & 7.30 & 6.50 & 6.50 & 6.85 & 8.85 \\
\hline 200 & - & - & - & - & - & - & 7.35 & 7.10 & 6.10 & 5.10 & 5.45 & 6.50 \\
\hline \multicolumn{13}{|l|}{$P M S B$} \\
\hline 20 & - & - & - & - & - & - & 5.45 & 4.80 & 7.10 & 7.60 & 9.50 & 14.60 \\
\hline 30 & - & - & - & - & - & - & 4.80 & 3.75 & 4.95 & 5.60 & 8.20 & 10.55 \\
\hline 50 & - & - & - & - & - & - & 5.50 & 5.10 & 4.55 & 4.45 & 6.10 & 7.15 \\
\hline 70 & - & - & - & - & - & - & 5.70 & 5.20 & 4.65 & 5.35 & 5.00 & 6.50 \\
\hline 100 & - & - & - & - & - & - & 6.10 & 4.60 & 4.90 & 5.60 & 4.40 & 5.15 \\
\hline 200 & - & - & - & - & - & - & 5.75 & 5.00 & 4.55 & 4.45 & 4.60 & 5.40 \\
\hline \multicolumn{13}{|l|}{$t_{b}^{*}$} \\
\hline 20 & - & - & - & - & - & - & 16.50 & 15.55 & 20.50 & 19.95 & 26.70 & 31.65 \\
\hline 30 & - & - & - & - & - & - & 12.30 & 12.40 & 16.80 & 15.60 & 20.10 & 27.70 \\
\hline 50 & - & - & - & - & - & - & 9.55 & 8.25 & 12.65 & 12.60 & 14.60 & 20.20 \\
\hline 70 & - & - & - & - & - & - & 8.40 & 7.75 & 8.70 & 9.90 & 12.65 & 14.30 \\
\hline 100 & - & - & - & - & - & - & 7.90 & 7.30 & 8.65 & 8.30 & 7.75 & 11.85 \\
\hline 200 & - & - & - & - & - & - & 7.70 & 7.25 & 6.50 & 7.00 & 6.30 & 7.35 \\
\hline
\end{tabular}

Notes: See notes to Table 2. The data generating process is the same as the one for Table 2, except $\rho_{i}=\rho=1$ and $\rho_{f 1}=\rho_{f 2}=0.3$. The $P_{b}, P M S B$, and $t_{b}^{*}$ tests adopt automatic lag-order selection for the estimation of long-run variances, which explains the empty boxes in the above table. 
Table 6: Power of Panel Unit Root Tests with Two Factors $(m=2)$, Serially Correlated $f_{t}$ and $\Delta v_{i x t}$, Intercept Only Case

\begin{tabular}{|c|c|c|c|c|c|c|c|c|c|c|c|c|}
\hline$(\mathrm{T}, \mathrm{N})$ & 20 & 30 & 50 & 70 & 100 & 200 & 20 & 30 & 50 & 70 & 100 & 200 \\
\hline $\operatorname{IPS}(p)$ & \multicolumn{6}{|c|}{$p=0$} & \multicolumn{6}{|c|}{$p=1$} \\
\hline 20 & 19.85 & 20.10 & 25.30 & 28.50 & 30.70 & 33.60 & 36.70 & 40.45 & 45.30 & 48.90 & 50.80 & 52.60 \\
\hline 30 & 20.00 & 22.45 & 26.45 & 29.35 & 31.95 & 36.50 & 41.25 & 44.60 & 51.90 & 52.25 & 54.70 & 59.35 \\
\hline 50 & 22.60 & 27.20 & 31.50 & 33.40 & 34.15 & 37.75 & 47.65 & 53.90 & 58.60 & 60.15 & 62.35 & 66.20 \\
\hline 70 & 26.90 & 29.95 & 37.00 & 41.50 & 41.25 & 46.90 & 55.10 & 60.25 & 64.80 & 69.40 & 71.15 & 73.35 \\
\hline 100 & 30.30 & 41.15 & 45.30 & 49.20 & 50.35 & 55.65 & 63.30 & 71.95 & 75.35 & 78.05 & 79.90 & 82.90 \\
\hline 200 & 62.55 & 74.75 & 79.90 & 83.15 & 82.85 & 86.00 & 91.15 & 94.60 & 96.45 & 96.75 & 98.00 & 97.75 \\
\hline $\operatorname{CIPS}(p)$ & \multicolumn{6}{|c|}{$p=0$} & \multicolumn{6}{|c|}{$p=1$} \\
\hline 20 & 9.80 & 10.85 & 13.20 & 14.15 & 13.05 & 14.00 & 8.10 & 8.10 & 9.35 & 8.35 & 8.30 & 8.95 \\
\hline 30 & 8.80 & 8.85 & 10.10 & 11.65 & 11.35 & 12.15 & 6.95 & 6.70 & 7.90 & 7.15 & 7.45 & 8.05 \\
\hline 50 & 11.80 & 17.90 & 23.15 & 21.90 & 19.30 & 27.75 & 9.05 & 13.55 & 16.05 & 15.15 & 11.80 & 17.35 \\
\hline 70 & 23.10 & 41.95 & 48.65 & 56.45 & 54.15 & 76.90 & 19.75 & 33.15 & 39.60 & 41.25 & 37.50 & 59.20 \\
\hline 100 & 54.45 & 85.20 & 92.90 & 96.20 & 96.95 & 99.70 & 49.05 & 77.75 & 87.20 & 91.25 & 93.20 & 99.05 \\
\hline 200 & 100.00 & 100.00 & 100.00 & 100.00 & 100.00 & 100.00 & 99.90 & 100.00 & 100.00 & 100.00 & 100.00 & 100.00 \\
\hline \multicolumn{13}{|l|}{$P_{b}$} \\
\hline 20 & - & - & - & - & - & - & 15.80 & 22.50 & 29.65 & 35.10 & 42.15 & 58.30 \\
\hline 30 & - & - & - & - & - & - & 22.50 & 39.60 & 49.60 & 53.50 & 59.00 & 65.15 \\
\hline 50 & - & - & - & - & - & - & 38.95 & 71.25 & 69.20 & 68.90 & 72.20 & 75.80 \\
\hline 70 & - & - & - & - & - & - & 55.40 & 84.60 & 79.40 & 77.85 & 79.90 & 82.40 \\
\hline 100 & - & - & - & - & - & - & 69.50 & 93.35 & 87.80 & 87.50 & 86.30 & 87.60 \\
\hline 200 & - & - & - & - & - & - & 89.85 & 99.45 & 98.05 & 97.30 & 97.15 & 97.85 \\
\hline \multicolumn{13}{|l|}{$P M S B$} \\
\hline 20 & - & - & - & - & - & - & 4.55 & 6.25 & 8.60 & 10.70 & 14.40 & 24.50 \\
\hline 30 & - & - & - & - & - & - & 8.40 & 16.55 & 21.70 & 27.35 & 33.90 & 46.80 \\
\hline 50 & - & - & - & - & - & - & 20.35 & 48.65 & 52.15 & 53.95 & 58.70 & 66.55 \\
\hline 70 & - & - & - & - & - & - & 34.80 & 70.90 & 67.60 & 66.85 & 71.10 & 74.70 \\
\hline 100 & - & - & - & - & - & - & 49.80 & 88.75 & 79.50 & 79.30 & 80.50 & 80.95 \\
\hline 200 & - & - & - & - & - & - & 81.05 & 99.20 & 96.10 & 94.85 & 95.60 & 95.65 \\
\hline \multicolumn{13}{|l|}{$t_{b}^{*}$} \\
\hline 20 & - & - & - & - & - & - & 63.30 & 80.25 & 80.45 & 79.35 & 81.40 & 85.35 \\
\hline 30 & - & - & - & - & - & - & 66.70 & 88.85 & 86.10 & 84.15 & 85.85 & 87.95 \\
\hline 50 & - & - & - & - & - & - & 77.50 & 95.15 & 91.55 & 89.55 & 92.20 & 92.70 \\
\hline 70 & - & - & - & - & - & - & 84.15 & 97.60 & 95.55 & 94.70 & 94.20 & 95.00 \\
\hline 100 & - & - & - & - & - & - & 90.05 & 99.40 & 98.20 & 96.95 & 97.15 & 98.05 \\
\hline 200 & - & - & - & - & - & - & 98.15 & 100.00 & 99.95 & 99.90 & 99.80 & 99.75 \\
\hline
\end{tabular}

Notes: See notes to Table 2. The data generating process is the same as the one for Table 2, except $\rho_{i} \sim i i d U[0.90,0.99]$ and $\rho_{f 1}=\rho_{f 2}=0.3$. The $P_{b}, P M S B$, and $t_{b}^{*}$ tests adopt automatic lag-order selection for the estimation of long-run variances, which explains the empty boxes in the above table. 
Table 7: Size of Panel Unit Root Tests, Supposing Three Factors Exist when There are Actually Two Factors $(m=2)$, Serially Correlated $\Delta v_{i x t}$, Intercept Only Case

\begin{tabular}{|c|c|c|c|c|c|c|c|c|c|c|c|c|}
\hline$(\mathrm{T}, \mathrm{N})$ & 20 & 30 & 50 & 70 & 100 & 200 & 20 & 30 & 50 & 70 & 100 & 200 \\
\hline $\operatorname{IPS}(p)$ & \multicolumn{6}{|c|}{$p=0$} & \multicolumn{6}{|c|}{$p=1$} \\
\hline 20 & 30.80 & 33.75 & 38.50 & 41.25 & 44.00 & 46.35 & 29.70 & 32.50 & 37.35 & 40.85 & 43.55 & 46.85 \\
\hline 30 & 30.85 & 34.40 & 39.45 & 43.35 & 44.45 & 47.95 & 31.45 & 35.80 & 38.70 & 42.00 & 43.60 & 48.30 \\
\hline 50 & 30.75 & 33.95 & 42.30 & 42.40 & 43.25 & 48.05 & 30.15 & 34.00 & 41.75 & 42.40 & 43.35 & 48.20 \\
\hline 70 & 33.60 & 37.90 & 40.60 & 43.05 & 43.70 & 47.50 & 33.80 & 37.25 & 41.35 & 43.45 & 43.70 & 47.65 \\
\hline 100 & 31.75 & 36.40 & 38.15 & 44.25 & 46.60 & 47.15 & 32.75 & 35.65 & 38.35 & 44.55 & 45.70 & 45.95 \\
\hline 200 & 33.90 & 37.10 & 39.20 & 43.80 & 44.35 & 49.00 & 33.25 & 36.40 & 39.85 & 44.55 & 44.40 & 48.95 \\
\hline $\operatorname{CIPS}(p)$ & \multicolumn{6}{|c|}{$p=0$} & \multicolumn{6}{|c|}{$p=1$} \\
\hline 20 & 8.65 & 8.60 & 10.10 & 9.30 & 8.75 & 10.50 & 7.10 & 8.30 & 8.45 & 7.75 & 7.30 & 6.70 \\
\hline 30 & 7.40 & 7.90 & 6.95 & 8.25 & 8.20 & 9.95 & 7.60 & 6.35 & 6.80 & 7.15 & 7.35 & 8.15 \\
\hline 50 & 5.25 & 7.35 & 7.25 & 6.95 & 7.35 & 7.30 & 5.05 & 6.85 & 6.25 & 5.90 & 5.80 & 6.40 \\
\hline 70 & 6.85 & 6.75 & 7.90 & 5.95 & 6.70 & 6.55 & 5.65 & 6.75 & 8.05 & 5.75 & 5.90 & 6.35 \\
\hline 100 & 5.85 & 4.80 & 5.60 & 6.30 & 6.45 & 6.85 & 4.85 & 4.40 & 5.35 & 5.80 & 5.70 & 6.30 \\
\hline 200 & 5.10 & 5.40 & 5.45 & 6.15 & 6.15 & 5.90 & 4.85 & 5.00 & 5.00 & 5.55 & 5.65 & 5.65 \\
\hline \multicolumn{13}{|l|}{$P_{b}$} \\
\hline 20 & - & - & - & - & - & - & 8.80 & 9.65 & 10.40 & 11.15 & 14.55 & 20.95 \\
\hline 30 & - & - & - & - & - & - & 8.55 & 8.35 & 9.00 & 8.80 & 10.90 & 14.10 \\
\hline 50 & - & - & - & - & - & - & 8.05 & 6.15 & 6.50 & 7.10 & 7.50 & 9.40 \\
\hline 70 & - & - & - & - & - & - & 8.60 & 7.80 & 7.00 & 6.25 & 6.35 & 7.15 \\
\hline 100 & - & - & - & - & - & - & 7.85 & 7.55 & 5.75 & 5.85 & 6.10 & 6.30 \\
\hline 200 & - & - & - & - & - & - & 8.45 & 7.30 & 6.10 & 7.35 & 5.15 & 5.80 \\
\hline \multicolumn{13}{|l|}{$P M S B$} \\
\hline 20 & - & - & - & - & - & - & 10.15 & 7.35 & 6.65 & 6.85 & 9.10 & 9.90 \\
\hline 30 & - & - & - & - & - & - & 9.20 & 8.85 & 6.90 & 6.90 & 7.45 & 8.20 \\
\hline 50 & - & - & - & - & - & - & 9.35 & 7.45 & 6.55 & 6.05 & 5.30 & 6.65 \\
\hline 70 & - & - & - & - & - & - & 9.75 & 8.20 & 6.45 & 5.70 & 6.05 & 4.75 \\
\hline 100 & - & - & - & - & - & - & 9.50 & 8.00 & 6.05 & 5.65 & 5.40 & 4.55 \\
\hline 200 & - & - & - & - & - & - & 10.20 & 8.25 & 6.95 & 6.45 & 4.95 & 4.70 \\
\hline \multicolumn{13}{|l|}{$t_{b}^{*}$} \\
\hline 20 & - & - & - & - & - & - & 31.60 & 35.60 & 38.80 & 46.15 & 47.75 & 51.45 \\
\hline 30 & - & - & - & - & - & - & 30.75 & 36.15 & 36.70 & 44.05 & 46.55 & 50.05 \\
\hline 50 & - & - & - & - & - & - & 31.20 & 34.80 & 38.35 & 42.40 & 47.55 & 51.75 \\
\hline 70 & - & - & - & - & - & - & 28.85 & 32.20 & 35.95 & 40.00 & 43.80 & 50.05 \\
\hline 100 & - & - & - & - & - & - & 26.85 & 29.35 & 34.05 & 40.10 & 42.20 & 50.15 \\
\hline 200 & - & - & - & - & - & - & 22.00 & 24.80 & 29.15 & 37.00 & 39.35 & 46.95 \\
\hline
\end{tabular}

Notes: See notes to Table 2. The data generating process is the same as the one for Table 2, except $\gamma_{i 2}=\gamma_{2}=0$ and $\gamma_{i x 1}=\gamma_{x 1}=0$. The CIPS test is based on cross section augmentation using $y_{i t}$ and $x_{i t}$. The $P_{b}, P M S B$, and $t_{b}^{*}$ tests are based on three extracted factors from the $y_{i t}$ series using principal components. These tests adopt automatic lag-order selection for the estimation of long-run variances, which explains the empty boxes in the above table. 
Table 8: Power of Panel Unit Root Tests, Supposing Three Factors Exist when There are Actually Two Factors $(m=2)$, Serially Correlated $\Delta v_{i x t}$, Intercept Only Case

\begin{tabular}{|c|c|c|c|c|c|c|c|c|c|c|c|c|}
\hline$(\mathrm{T}, \mathrm{N})$ & 20 & 30 & 50 & 70 & 100 & 200 & 20 & 30 & 50 & 70 & 100 & 200 \\
\hline $\operatorname{IPS}(p)$ & \multicolumn{6}{|c|}{$p=0$} & \multicolumn{6}{|c|}{$p=1$} \\
\hline 20 & 37.00 & 39.85 & 46.55 & 48.25 & 52.50 & 54.25 & 36.00 & 40.35 & 46.75 & 46.60 & 51.80 & 53.90 \\
\hline 30 & 41.30 & 46.45 & 52.00 & 54.50 & 57.75 & 61.30 & 39.10 & 45.60 & 51.85 & 53.85 & 56.50 & 61.60 \\
\hline 50 & 48.70 & 55.45 & 65.55 & 62.65 & 65.40 & 68.65 & 47.60 & 55.65 & 64.25 & 61.90 & 64.65 & 68.70 \\
\hline 70 & 59.35 & 66.75 & 72.70 & 73.60 & 74.20 & 78.25 & 59.25 & 65.60 & 72.70 & 72.70 & 72.50 & 77.60 \\
\hline 100 & 69.95 & 77.95 & 82.50 & 83.45 & 85.50 & 87.70 & 69.15 & 76.95 & 82.20 & 82.35 & 84.30 & 86.95 \\
\hline 200 & 95.20 & 97.55 & 98.55 & 98.30 & 98.85 & 99.70 & 94.60 & 97.00 & 98.35 & 97.75 & 98.25 & 99.30 \\
\hline $\operatorname{CIPS}(p)$ & \multicolumn{6}{|c|}{$p=0$} & \multicolumn{6}{|c|}{$p=1$} \\
\hline 20 & 9.35 & 9.25 & 9.65 & 9.70 & 9.05 & 11.50 & 7.65 & 8.80 & 7.55 & 7.80 & 7.00 & 7.30 \\
\hline 30 & 8.70 & 9.70 & 7.65 & 10.10 & 9.00 & 11.95 & 7.40 & 7.65 & 7.20 & 7.25 & 7.90 & 9.25 \\
\hline 50 & 10.90 & 14.05 & 14.45 & 16.40 & 17.55 & 21.40 & 9.65 & 12.15 & 10.55 & 12.80 & 12.65 & 15.35 \\
\hline 70 & 20.55 & 26.00 & 32.10 & 32.30 & 35.80 & 46.30 & 14.95 & 21.00 & 25.30 & 24.60 & 25.75 & 33.90 \\
\hline 100 & 41.00 & 55.35 & 74.15 & 78.55 & 85.00 & 94.10 & 35.45 & 47.30 & 63.10 & 66.90 & 74.35 & 87.00 \\
\hline 200 & 99.00 & 100.00 & 100.00 & 100.00 & 100.00 & 100.00 & 98.15 & 99.80 & 100.00 & 100.00 & 100.00 & 100.00 \\
\hline \multicolumn{13}{|l|}{$P_{b}$} \\
\hline 20 & - & - & - & - & - & - & 18.90 & 23.90 & 35.50 & 40.15 & 55.30 & 77.40 \\
\hline 30 & - & - & - & - & - & - & 31.35 & 39.90 & 61.50 & 65.50 & 76.35 & 91.05 \\
\hline 50 & - & - & - & - & - & - & 58.10 & 70.65 & 85.55 & 87.95 & 92.15 & 98.30 \\
\hline 70 & - & - & - & - & - & - & 77.80 & 83.75 & 94.45 & 92.90 & 96.85 & 99.00 \\
\hline 100 & - & - & - & - & - & - & 91.20 & 92.90 & 97.45 & 97.60 & 99.20 & 99.85 \\
\hline 200 & - & - & - & - & - & - & 99.40 & 99.15 & 99.95 & 99.95 & 100.00 & 100.00 \\
\hline \multicolumn{13}{|l|}{$P M S B$} \\
\hline 20 & - & - & - & - & - & - & 15.85 & 15.15 & 18.70 & 20.60 & 28.25 & 48.85 \\
\hline 30 & - & - & - & - & - & - & 25.85 & 29.40 & 44.35 & 46.50 & 58.50 & 81.05 \\
\hline 50 & - & - & - & - & - & - & 52.15 & 60.50 & 78.80 & 82.50 & 88.20 & 97.15 \\
\hline 70 & - & - & - & - & - & - & 72.00 & 79.15 & 91.65 & 90.65 & 95.70 & 99.00 \\
\hline 100 & - & - & - & - & - & - & 86.95 & 91.05 & 96.20 & 96.90 & 98.95 & 99.95 \\
\hline 200 & - & - & - & - & - & - & 99.35 & 99.15 & 99.95 & 99.90 & 100.00 & 100.00 \\
\hline \multicolumn{13}{|l|}{$t_{b}^{*}$} \\
\hline 20 & - & - & - & - & - & - & 84.65 & 87.95 & 92.30 & 92.45 & 95.15 & 96.05 \\
\hline 30 & - & - & - & - & - & - & 90.90 & 91.95 & 95.95 & 95.20 & 97.05 & 98.70 \\
\hline 50 & - & - & - & - & - & - & 96.05 & 96.55 & 98.95 & 98.70 & 99.30 & 99.55 \\
\hline 70 & - & - & - & - & - & - & 98.65 & 98.70 & 99.75 & 99.35 & 99.75 & 99.90 \\
\hline 100 & - & - & - & - & - & - & 99.85 & 99.50 & 100.00 & 99.95 & 100.00 & 100.00 \\
\hline 200 & - & - & - & - & - & - & 100.00 & 100.00 & 100.00 & 100.00 & 100.00 & 100.00 \\
\hline
\end{tabular}

Notes: See notes to Table 2. The data generating process is the same as the one for Table 2 , except $\gamma_{i 2}=\gamma_{2}=0$ and $\gamma_{i x 1}=\gamma_{x 1}=0$. The CIPS test is based on cross section augmentation using $y_{i t}$ and $x_{i t}$. The $P_{b}$, $P M S B$, and $t_{b}^{*}$ tests are based on three extracted factors from the $y_{i t}$ series using principal components. These tests adopt automatic lag-order selection for the estimation of long-run variances, which explains the empty boxes in the above table. 
Table 9: Size of Panel Unit Root Tests, Supposing Three Factors Exist when There are Actually Two Factors $(m=2)$, Serially Correlated $\Delta v_{i x t}$, Intercept Only Case. For the $P_{b}, P M S B$, and $t_{b}^{*}$ tests the Number of Factors is Estimated.

\begin{tabular}{|c|c|c|c|c|c|c|c|c|c|c|c|c|}
\hline$(\mathrm{T}, \mathrm{N})$ & 20 & 30 & 50 & 70 & 100 & 200 & 20 & 30 & 50 & 70 & 100 & 200 \\
\hline $\operatorname{IPS}(p)$ & \multicolumn{6}{|c|}{$p=0$} & \multicolumn{6}{|c|}{$p=1$} \\
\hline 20 & 30.80 & 33.75 & 38.50 & 41.25 & 44.00 & 46.35 & 29.70 & 32.50 & 37.35 & 40.85 & 43.55 & 46.85 \\
\hline 30 & 30.85 & 34.40 & 39.45 & 43.35 & 44.45 & 47.95 & 31.45 & 35.80 & 38.70 & 42.00 & 43.60 & 48.30 \\
\hline 50 & 30.75 & 33.95 & 42.30 & 42.40 & 43.25 & 48.05 & 30.15 & 34.00 & 41.75 & 42.40 & 43.35 & 48.20 \\
\hline 70 & 33.60 & 37.90 & 40.60 & 43.05 & 43.70 & 47.50 & 33.80 & 37.25 & 41.35 & 43.45 & 43.70 & 47.65 \\
\hline 100 & 31.75 & 36.40 & 38.15 & 44.25 & 46.60 & 47.15 & 32.75 & 35.65 & 38.35 & 44.55 & 45.70 & 45.95 \\
\hline 200 & 33.90 & 37.10 & 39.20 & 43.80 & 44.35 & 49.00 & 33.25 & 36.40 & 39.85 & 44.55 & 44.40 & 48.95 \\
\hline $\operatorname{CIPS}(p)$ & \multicolumn{6}{|c|}{$p=0$} & \multicolumn{6}{|c|}{$p=1$} \\
\hline 20 & 8.65 & 8.60 & 10.10 & 9.30 & 8.75 & 10.50 & 7.10 & 8.30 & 8.45 & 7.75 & 7.30 & 6.70 \\
\hline 30 & 7.40 & 7.90 & 6.95 & 8.25 & 8.20 & 9.95 & 7.60 & 6.35 & 6.80 & 7.15 & 7.35 & 8.15 \\
\hline 50 & 5.25 & 7.35 & 7.25 & 6.95 & 7.35 & 7.30 & 5.05 & 6.85 & 6.25 & 5.90 & 5.80 & 6.40 \\
\hline 70 & 6.85 & 6.75 & 7.90 & 5.95 & 6.70 & 6.55 & 5.65 & 6.75 & 8.05 & 5.75 & 5.90 & 6.35 \\
\hline 100 & 5.85 & 4.80 & 5.60 & 6.30 & 6.45 & 6.85 & 4.85 & 4.40 & 5.35 & 5.80 & 5.70 & 6.30 \\
\hline 200 & 5.10 & 5.40 & 5.45 & 6.15 & 6.15 & 5.90 & 4.85 & 5.00 & 5.00 & 5.55 & 5.65 & 5.65 \\
\hline \multicolumn{13}{|l|}{$P_{b}$} \\
\hline 20 & - & - & - & - & - & & 9.25 & 8.00 & 10.15 & 12.95 & 15.45 & 28.45 \\
\hline 30 & - & - & - & - & - & & 9.50 & 9.20 & 8.20 & 9.40 & 10.05 & 19.90 \\
\hline 50 & - & - & - & - & - & & 10.75 & 7.75 & 6.35 & 6.85 & 7.65 & 9.45 \\
\hline 70 & - & - & - & - & - & & 11.50 & 7.95 & 7.25 & 6.15 & 6.65 & 6.95 \\
\hline 100 & - & - & - & - & - & & 10.25 & 7.50 & 5.70 & 6.10 & 6.15 & 6.40 \\
\hline 200 & - & - & - & - & - & & 11.65 & 7.50 & 6.25 & 7.10 & 5.05 & 5.65 \\
\hline \multicolumn{13}{|l|}{$P M S B$} \\
\hline 20 & - & - & - & - & - & & 0.70 & 1.85 & 2.20 & 1.35 & 2.20 & 1.25 \\
\hline 30 & - & - & - & - & - & & 0.85 & 2.70 & 3.05 & 2.05 & 2.90 & 2.40 \\
\hline 50 & - & - & - & - & - & & 1.25 & 3.25 & 3.20 & 2.80 & 2.65 & 3.05 \\
\hline 70 & - & - & - & - & - & & 1.20 & 3.80 & 3.60 & 3.35 & 3.50 & 2.65 \\
\hline 100 & - & - & - & - & - & & 0.95 & 3.70 & 3.40 & 3.50 & 3.55 & 3.30 \\
\hline 200 & - & - & - & - & - & & 1.05 & 5.05 & 5.40 & 4.20 & 3.35 & 3.40 \\
\hline \multicolumn{13}{|l|}{$t_{b}^{*}$} \\
\hline 20 & - & - & - & - & - & & 8.80 & 11.85 & 14.65 & 19.10 & 20.90 & 29.20 \\
\hline 30 & - & - & - & - & - & & 9.75 & 12.70 & 10.95 & 15.00 & 18.75 & 26.60 \\
\hline 50 & - & - & - & - & - & & 9.60 & 10.90 & 9.95 & 12.65 & 12.80 & 20.35 \\
\hline 70 & - & - & - & - & - & & 11.30 & 10.10 & 8.30 & 10.35 & 10.30 & 16.30 \\
\hline 100 & - & - & - & - & - & & 10.90 & 8.15 & 8.80 & 9.65 & 10.20 & 13.75 \\
\hline 200 & - & - & - & - & - & & 11.00 & 8.25 & 7.45 & 8.20 & 8.10 & 9.85 \\
\hline
\end{tabular}

Notes: See notes to Table 2. The data generating process is the same as the one for Table 2 , except $\gamma_{i 2}=\gamma_{2}=0$ and $\gamma_{i x 1}=\gamma_{x 1}=0$. The CIPS test is based on cross section augmentation using $y_{i t}$ and $x_{i t}$. For the $P_{b}, P M S B$, and $t_{b}^{*}$ tests, the number of factors is determined by the BIC3 criterion of Bai and $\mathrm{Ng}(2002)$ with the maximum number set to three, and the factors are extracted from the $y_{i t}$ series using principal components. These tests adopt automatic lag-order selection for the estimation of long-run variances, which explains the empty boxes in the above table. 
Table 10: Power of Panel Unit Root Tests, Supposing Three Factors Exist when There are Actually Two Factors $(m=2)$, Serially Correlated $\Delta v_{i x t}$, Intercept Only Case. For the $P_{b}, P M S B$, and $t_{b}^{*}$ tests the Number of Factors is Estimated.

\begin{tabular}{|c|c|c|c|c|c|c|c|c|c|c|c|c|}
\hline$(\mathrm{T}, \mathrm{N})$ & 20 & 30 & 50 & 70 & 100 & 200 & 20 & 30 & 50 & 70 & 100 & 200 \\
\hline $\operatorname{IPS}(p)$ & \multicolumn{6}{|c|}{$p=0$} & \multicolumn{6}{|c|}{$p=1$} \\
\hline 20 & 37.00 & 39.85 & 46.55 & 48.25 & 52.50 & 54.25 & 36.00 & 40.35 & 46.75 & 46.60 & 51.80 & 53.90 \\
\hline 30 & 41.30 & 46.45 & 52.00 & 54.50 & 57.75 & 61.30 & 39.10 & 45.60 & 51.85 & 53.85 & 56.50 & 61.60 \\
\hline 50 & 48.70 & 55.45 & 65.55 & 62.65 & 65.40 & 68.65 & 47.60 & 55.65 & 64.25 & 61.90 & 64.65 & 68.70 \\
\hline 70 & 59.35 & 66.75 & 72.70 & 73.60 & 74.20 & 78.25 & 59.25 & 65.60 & 72.70 & 72.70 & 72.50 & 77.60 \\
\hline 100 & 69.95 & 77.95 & 82.50 & 83.45 & 85.50 & 87.70 & 69.15 & 76.95 & 82.20 & 82.35 & 84.30 & 86.95 \\
\hline 200 & 95.20 & 97.55 & 98.55 & 98.30 & 98.85 & 99.70 & 94.60 & 97.00 & 98.35 & 97.75 & 98.25 & 99.30 \\
\hline $\operatorname{CIPS}(p)$ & \multicolumn{6}{|c|}{$p=0$} & \multicolumn{6}{|c|}{$p=1$} \\
\hline 20 & 9.35 & 9.25 & 9.65 & 9.70 & 9.05 & 11.50 & 7.65 & 8.80 & 7.55 & 7.80 & 7.00 & 7.30 \\
\hline 30 & 8.70 & 9.70 & 7.65 & 10.10 & 9.00 & 11.95 & 7.40 & 7.65 & 7.20 & 7.25 & 7.90 & 9.25 \\
\hline 50 & 10.90 & 14.05 & 14.45 & 16.40 & 17.55 & 21.40 & 9.65 & 12.15 & 10.55 & 12.80 & 12.65 & 15.35 \\
\hline 70 & 20.55 & 26.00 & 32.10 & 32.30 & 35.80 & 46.30 & 14.95 & 21.00 & 25.30 & 24.60 & 25.75 & 33.90 \\
\hline 100 & 41.00 & 55.35 & 74.15 & 78.55 & 85.00 & 94.10 & 35.45 & 47.30 & 63.10 & 66.90 & 74.35 & 87.00 \\
\hline 200 & 99.00 & 100.00 & 100.00 & 100.00 & 100.00 & 100.00 & 98.15 & 99.80 & 100.00 & 100.00 & 100.00 & 100.00 \\
\hline \multicolumn{13}{|l|}{$P_{b}$} \\
\hline 20 & - & - & - & - & - & - & 27.40 & 32.55 & 46.15 & 49.45 & 59.45 & 72.25 \\
\hline 30 & - & - & - & - & - & - & 43.05 & 48.70 & 64.55 & 66.50 & 72.25 & 79.75 \\
\hline 50 & - & - & - & - & - & - & 69.95 & 72.60 & 86.10 & 83.95 & 87.35 & 88.90 \\
\hline 70 & - & - & - & - & - & - & 85.25 & 84.05 & 93.95 & 89.20 & 91.90 & 91.95 \\
\hline 100 & - & - & - & - & - & - & 94.35 & 91.75 & 96.15 & 95.10 & 96.55 & 95.60 \\
\hline 200 & - & - & - & - & - & - & 99.80 & 98.85 & 99.85 & 99.50 & 99.90 & 99.80 \\
\hline \multicolumn{13}{|l|}{$P M S B$} \\
\hline 20 & - & - & - & - & - & - & 2.40 & 4.55 & 10.55 & 7.80 & 13.75 & 21.55 \\
\hline 30 & - & - & - & - & - & - & 4.95 & 14.15 & 33.30 & 30.60 & 44.05 & 54.80 \\
\hline 50 & - & - & - & - & - & - & 18.00 & 45.35 & 72.40 & 71.65 & 78.00 & 81.70 \\
\hline 70 & - & - & - & - & - & - & 32.35 & 67.15 & 88.25 & 82.40 & 87.10 & 87.30 \\
\hline 100 & - & - & - & - & - & - & 58.35 & 84.95 & 93.65 & 91.50 & 93.70 & 92.70 \\
\hline 200 & - & - & - & - & - & - & 95.90 & 97.70 & 99.95 & 98.60 & 99.60 & 99.40 \\
\hline \multicolumn{13}{|l|}{$t_{b}^{*}$} \\
\hline 20 & - & - & - & - & - & - & 77.60 & 81.05 & 88.65 & 87.95 & 91.60 & 93.55 \\
\hline 30 & - & - & - & - & - & - & 87.50 & 88.50 & 93.75 & 90.35 & 94.95 & 95.60 \\
\hline 50 & - & - & - & - & - & - & 96.05 & 93.50 & 97.75 & 96.30 & 97.50 & 97.80 \\
\hline 70 & - & - & - & - & - & - & 98.85 & 96.80 & 99.25 & 97.50 & 98.80 & 98.65 \\
\hline 100 & - & - & - & - & - & - & 99.90 & 99.10 & 99.95 & 99.75 & 99.85 & 99.60 \\
\hline 200 & - & - & - & - & - & - & 100.00 & 99.85 & 100.00 & 100.00 & 100.00 & 100.00 \\
\hline
\end{tabular}

Notes: See notes to Table 2. The data generating process is the same as the one for Table 2 , except $\gamma_{i 2}=\gamma_{2}=0$ and $\gamma_{i x 1}=\gamma_{x 1}=0$. The CIPS test is based on cross section augmentation using $y_{i t}$ and $x_{i t}$. For the $P_{b}, P M S B$, and $t_{b}^{*}$ tests, the number of factors is determined by the BIC3 criterion of Bai and $\mathrm{Ng}(2002)$ with the maximum number set to three, and the factors are extracted from the $y_{i t}$ series using principal components. These tests adopt automatic lag-order selection for the estimation of long-run variances, which explains the empty boxes in the above table. 
Table 11: Size and Power of Panel Unit Root Tests with Two Factors $(m=2)$, Serially Correlated $\Delta v_{i x t}$, Intercept and Linear Trend Case

\begin{tabular}{|c|c|c|c|c|c|c|c|c|c|c|c|c|}
\hline & \multicolumn{6}{|c|}{ Size: $\rho_{i}=\rho=1$} & \multicolumn{6}{|c|}{ Power: $\rho_{i} \sim$ iidU $[0.90,0.99]$} \\
\hline$(\mathrm{T}, \mathrm{N})$ & 20 & 30 & 50 & 70 & 100 & 200 & 20 & 30 & 50 & 70 & 100 & 200 \\
\hline $\operatorname{IPS}(p)$ & \multicolumn{6}{|c|}{$p=0$} & \multicolumn{6}{|c|}{$p=0$} \\
\hline 20 & 32.40 & 33.25 & 38.15 & 38.30 & 41.05 & 44.70 & 34.10 & 34.55 & 39.20 & 40.25 & 42.65 & 46.40 \\
\hline 30 & 32.70 & 34.50 & 39.40 & 40.05 & 41.75 & 45.75 & 34.55 & 38.20 & 42.00 & 43.85 & 44.80 & 48.75 \\
\hline 50 & 34.70 & 35.80 & 37.15 & 39.60 & 44.95 & 47.10 & 40.20 & 43.20 & 46.25 & 48.20 & 52.40 & 55.25 \\
\hline 70 & 32.20 & 35.80 & 40.55 & 41.25 & 44.45 & 46.35 & 42.65 & 48.05 & 55.40 & 56.30 & 58.65 & 61.55 \\
\hline 100 & 30.85 & 36.40 & 40.75 & 41.25 & 45.30 & 45.60 & 50.65 & 58.25 & 63.45 & 65.30 & 66.45 & 70.65 \\
\hline 200 & 32.70 & 36.45 & 39.45 & 42.05 & 44.20 & 46.00 & 78.40 & 83.75 & 88.15 & 90.90 & 91.05 & 94.05 \\
\hline $\operatorname{CIPS}(p)$ & \multicolumn{6}{|c|}{$p=0$} & \multicolumn{6}{|c|}{$p=0$} \\
\hline 20 & 4.45 & 5.00 & 5.15 & 4.40 & 4.70 & 6.15 & 4.45 & 4.50 & 4.35 & 4.25 & 4.40 & 4.50 \\
\hline 30 & 4.30 & 3.60 & 3.85 & 4.70 & 3.90 & 4.50 & 4.85 & 3.80 & 4.60 & 4.30 & 3.85 & 3.25 \\
\hline 50 & 4.95 & 4.50 & 4.65 & 5.55 & 4.25 & 4.65 & 7.05 & 6.75 & 6.40 & 6.30 & 5.80 & 5.65 \\
\hline 70 & 4.15 & 4.90 & 4.50 & 4.60 & 4.30 & 4.80 & 8.15 & 13.10 & 14.25 & 14.85 & 15.00 & 18.20 \\
\hline 100 & 5.75 & 5.10 & 4.15 & 4.50 & 4.60 & 5.80 & 22.30 & 32.90 & 43.90 & 49.20 & 55.65 & 71.90 \\
\hline 200 & 4.80 & 5.75 & 5.00 & 5.80 & 3.90 & 5.55 & 93.95 & 99.75 & 99.95 & 100.00 & 100.00 & 100.00 \\
\hline \multicolumn{13}{|l|}{$P_{b}$} \\
\hline 20 & 71.95 & 81.65 & 91.70 & 96.70 & 98.70 & 99.80 & 71.70 & 81.15 & 91.70 & 96.05 & 98.30 & 99.55 \\
\hline 30 & 56.35 & 67.15 & 82.20 & 89.30 & 96.05 & 99.85 & 55.00 & 69.20 & 82.65 & 87.40 & 93.70 & 97.20 \\
\hline 50 & 35.00 & 42.30 & 56.10 & 69.50 & 77.95 & 95.45 & 36.70 & 47.95 & 62.55 & 69.60 & 76.30 & 85.75 \\
\hline 70 & 24.80 & 29.70 & 39.85 & 50.80 & 58.65 & 82.10 & 29.15 & 33.60 & 49.25 & 57.30 & 63.85 & 76.85 \\
\hline 100 & 17.30 & 19.10 & 28.90 & 30.80 & 43.35 & 62.70 & 21.50 & 27.40 & 41.25 & 47.75 & 52.70 & 67.60 \\
\hline 200 & 10.50 & 11.65 & 13.10 & 16.05 & 18.15 & 26.30 & 15.70 & 19.30 & 26.95 & 32.55 & 38.60 & 52.75 \\
\hline \multicolumn{13}{|l|}{$P M S B$} \\
\hline 20 & 0.00 & 0.05 & 0.10 & 0.15 & 0.15 & 0.55 & 0.00 & 0.00 & 0.15 & 0.00 & 0.05 & 0.35 \\
\hline 30 & 0.45 & 0.30 & 0.20 & 0.15 & 0.25 & 0.55 & 0.45 & 0.55 & 0.35 & 0.40 & 0.40 & 0.45 \\
\hline 50 & 1.05 & 1.05 & 1.40 & 1.00 & 0.50 & 0.45 & 2.45 & 4.10 & 6.80 & 6.50 & 9.30 & 18.30 \\
\hline 70 & 1.85 & 2.00 & 1.30 & 1.65 & 1.15 & 0.75 & 6.35 & 10.90 & 24.20 & 26.70 & 32.85 & 51.60 \\
\hline 100 & 2.00 & 1.90 & 1.95 & 2.00 & 1.55 & 1.25 & 14.75 & 30.90 & 57.15 & 55.15 & 59.35 & 71.75 \\
\hline 200 & 2.95 & 3.00 & 2.70 & 2.55 & 2.95 & 2.70 & 50.60 & 78.50 & 93.65 & 89.05 & 87.50 & 91.50 \\
\hline \multicolumn{13}{|l|}{$t_{b}^{*}$} \\
\hline 20 & 94.50 & 94.40 & 97.50 & 98.70 & 99.00 & 99.45 & 94.00 & 94.10 & 97.35 & 98.60 & 98.75 & 99.35 \\
\hline 30 & 79.30 & 79.85 & 90.20 & 93.35 & 96.75 & 98.25 & 78.90 & 80.65 & 89.90 & 92.75 & 95.30 & 96.65 \\
\hline 50 & 46.20 & 52.65 & 66.30 & 76.75 & 83.10 & 92.35 & 46.00 & 53.20 & 66.90 & 75.15 & 77.80 & 85.45 \\
\hline 70 & 27.60 & 32.95 & 47.30 & 57.25 & 65.65 & 83.40 & 29.35 & 33.45 & 49.90 & 55.70 & 61.50 & 75.15 \\
\hline 100 & 18.35 & 21.60 & 30.85 & 35.00 & 46.25 & 65.30 & 19.05 & 24.20 & 36.50 & 41.85 & 46.60 & 61.15 \\
\hline 200 & 9.90 & 11.25 & 12.30 & 15.60 & 17.75 & 28.50 & 11.95 & 14.15 & 19.05 & 23.30 & 29.10 & 39.30 \\
\hline
\end{tabular}

Notes: $y_{i t}$ is generated as $y_{i t}=\alpha_{i}+\left(1-\rho_{i}\right) \delta_{i} t+\rho_{i} y_{i, t-1}+\gamma_{i 1} f_{1 t}+\gamma_{i 2} f_{2 t}+\varepsilon_{i t}, i=1,2, \ldots, N ; t=-49, \ldots, T$ with $y_{i,-50}=0$, where $\rho_{i}=\rho=1$ for size and $\rho_{i} \sim i i d U[0.90,0.99]$ for power; $\alpha_{i}$ and $\delta_{i} \sim i i d[0.0,0.02] ; f_{j t}=\rho_{f j} f_{f j, t-1}+\varpi_{j t}$, $\varpi_{j t} \sim \operatorname{iidN}(0,1)$ with $f_{j,-50}=0$ and $\rho_{f j}=0$ for $j=1,2 ; \varepsilon_{i t}=\rho_{i \varepsilon} \varepsilon_{i t-1}+\zeta_{i t}, \zeta_{i t} \sim i i d N\left(0, \sigma_{i}^{2}\right)$ with $\varepsilon_{i,-50}=0$ and $\rho_{i \varepsilon}=\rho_{\varepsilon}=0, \sigma_{i}^{2} \sim \operatorname{iidU}[0.5,1.5] . \quad x_{i t}$ is generated as $x_{i t}=\mu_{i}+\lambda_{i} t+\gamma_{i x 1} f_{1 t}+\gamma_{i x 2} f_{2 t}+v_{i x t}, i=1,2, \ldots, N$; $t=-49, \ldots, T$ with $x_{i,-50}=0$, where $\mu_{i} \sim \operatorname{iidN}(1,1) ; \lambda_{i} \sim i i d[0.0,0.02] ; v_{i x t}=v_{i x t-1}+e_{i v x t}, e_{i v x t}=\rho_{i v x} e_{i v x, t-1}+\varrho_{i t}$, $\varrho_{i t} \sim i i d N(0,1)$ with $v_{i x,-50}=0, e_{i v x,-50}=0$, and $\rho_{i v x} \sim i i d U[0.2,0.4]$. Factor loadings are generated as $\gamma_{i 1} \sim i i d U[1,3]$, $\gamma_{i 2} \sim \operatorname{iidU}[0,2], \gamma_{i x 1} \sim \operatorname{iidU}[0,2], \gamma_{i x 2} \sim \operatorname{iidU[1,3]}$, so that the rank condition (9) is satisfied. $\alpha_{i}, \gamma_{i 1}, \gamma_{i 2}, \rho_{f j}, \rho_{i}, \mu_{i}$, $\gamma_{i x 1}, \gamma_{i x 2}, \rho_{i v x}, \delta_{i}$ and $\lambda_{i}$ are drawn once and fixed over the replications. The IPS(p) test is the Im et al. (2003) panel unit root test with lag-augmentation of order $p$. The CIPS(p) test is the proposed panel unit root test, defined by (35), based on cross section augmentation using $y_{i t}, x_{i 1 t}$ and,$x_{i 2 t}(k=2)$ with lag-augmentation of order $p$. The $P_{b}$ and $P M S B$ tests are the Bai and $\mathrm{Ng}(2007)$ pooled panel unit root tests for the idiosyncratic errors. The $t_{b}^{*}$ test is the Moon and Perron (2004) panel unit root test for the idiosyncratic errors. The $P_{b}, P M S B$, and $t_{b}^{*}$ tests are based on two extracted factors from the $y_{i t}$ series using principal components, and they adopt automatic lag-order selection for the estimation of long-run variances following Andrews and Monahan (1992). All tests are conducted at the 5\% significance level, and the CIPS(p) test is based on critical values for different $p$ and $k$. All experiments are based on 2000 replications. 
Table 12: Size and Power of Panel Unit Root Tests, Positive Serially Correlated $\varepsilon_{i t}$ and $\Delta v_{i x t}$, Intercept and Linear Trend Case

\begin{tabular}{|c|c|c|c|c|c|c|c|c|c|c|c|c|}
\hline & \multicolumn{6}{|c|}{ Size: $\rho_{i}=\rho=1$} & \multicolumn{6}{|c|}{ Power: $\rho_{i} \sim$ iidU $[0.90,0.99]$} \\
\hline$(\mathrm{T}, \mathrm{N})$ & 20 & 30 & 50 & 70 & 100 & 200 & 20 & 30 & 50 & 70 & 100 & 200 \\
\hline $\operatorname{IPS}(p)$ & \multicolumn{6}{|c|}{$p=1$} & \multicolumn{6}{|c|}{$p=1$} \\
\hline 20 & 26.60 & 30.75 & 36.95 & 35.80 & 40.00 & 43.00 & 28.25 & 32.35 & 38.85 & 36.95 & 41.95 & 44.90 \\
\hline 30 & 28.05 & 32.30 & 35.40 & 35.75 & 39.30 & 43.80 & 29.90 & 35.15 & 38.35 & 38.65 & 42.90 & 46.60 \\
\hline 50 & 26.75 & 31.75 & 31.90 & 37.25 & 39.55 & 43.55 & 33.50 & 38.35 & 41.35 & 47.00 & 49.05 & 53.85 \\
\hline 70 & 27.85 & 31.45 & 35.40 & 39.20 & 40.75 & 43.50 & 38.40 & 45.75 & 51.40 & 52.85 & 54.80 & 58.55 \\
\hline 100 & 27.15 & 33.05 & 34.90 & 36.60 & 42.20 & 42.85 & 47.80 & 56.15 & 61.15 & 63.70 & 66.45 & 69.25 \\
\hline 200 & 26.00 & 30.30 & 33.55 & 36.80 & 40.30 & 41.70 & 76.05 & 85.50 & 88.20 & 89.45 & 91.55 & 93.10 \\
\hline $\operatorname{CIPS}(p)$ & \multicolumn{6}{|c|}{$p=1$} & \multicolumn{6}{|c|}{$p=1$} \\
\hline 20 & 3.20 & 3.70 & 2.70 & 3.00 & 2.25 & 1.80 & 3.05 & 3.35 & 2.20 & 3.25 & 2.00 & 1.80 \\
\hline 30 & 3.40 & 2.65 & 2.90 & 2.85 & 3.00 & 3.60 & 3.60 & 3.20 & 2.75 & 3.40 & 2.55 & 3.95 \\
\hline 50 & 4.15 & 3.85 & 3.40 & 4.65 & 2.95 & 4.15 & 5.40 & 5.85 & 6.25 & 5.85 & 5.90 & 6.40 \\
\hline 70 & 4.15 & 3.60 & 4.75 & 4.15 & 4.20 & 3.85 & 7.90 & 10.30 & 13.25 & 11.85 & 11.95 & 13.25 \\
\hline 100 & 4.45 & 3.90 & 4.10 & 4.35 & 4.25 & 3.60 & 18.60 & 22.50 & 34.20 & 33.90 & 38.25 & 48.20 \\
\hline 200 & 5.05 & 4.75 & 4.75 & 4.95 & 4.20 & 4.60 & 84.50 & 96.95 & 99.85 & 99.90 & 100.00 & 100.00 \\
\hline \multicolumn{13}{|l|}{$P_{b}$} \\
\hline 20 & 33.15 & 35.60 & 43.75 & 48.60 & 59.05 & 70.10 & 33.50 & 35.75 & 45.05 & 49.20 & 61.25 & 71.40 \\
\hline 30 & 21.45 & 23.75 & 27.70 & 31.20 & 41.05 & 54.40 & 21.80 & 23.75 & 29.55 & 34.25 & 44.00 & 57.35 \\
\hline 50 & 12.95 & 13.45 & 17.20 & 19.80 & 22.85 & 33.15 & 12.70 & 14.45 & 19.40 & 22.30 & 29.35 & 42.00 \\
\hline 70 & 11.05 & 11.30 & 12.55 & 13.20 & 16.20 & 22.75 & 10.90 & 11.75 & 14.05 & 17.60 & 24.85 & 33.75 \\
\hline 100 & 8.70 & 9.80 & 10.20 & 10.35 & 11.65 & 16.00 & 8.75 & 9.05 & 10.35 & 14.95 & 18.60 & 28.35 \\
\hline 200 & 7.50 & 6.85 & 7.00 & 6.55 & 8.55 & 8.90 & 5.45 & 5.80 & 7.10 & 11.50 & 16.35 & 21.55 \\
\hline \multicolumn{13}{|l|}{$P M S B$} \\
\hline 20 & 0.20 & 0.35 & 0.45 & 0.65 & 0.65 & 1.50 & 0.15 & 0.25 & 0.55 & 0.70 & 0.80 & 1.05 \\
\hline 30 & 0.70 & 0.30 & 0.35 & 0.20 & 0.40 & 0.80 & 0.40 & 0.60 & 0.75 & 0.55 & 0.75 & 1.05 \\
\hline 50 & 0.70 & 0.70 & 0.55 & 0.90 & 0.35 & 0.30 & 1.50 & 3.65 & 5.70 & 6.10 & 8.60 & 18.30 \\
\hline 70 & 0.80 & 1.00 & 1.20 & 0.70 & 0.65 & 0.75 & 6.45 & 13.45 & 27.60 & 30.30 & 40.00 & 61.85 \\
\hline 100 & 1.55 & 1.45 & 1.60 & 1.35 & 1.70 & 0.90 & 18.10 & 39.90 & 68.40 & 70.25 & 75.55 & 85.00 \\
\hline 200 & 2.45 & 3.00 & 2.20 & 2.20 & 2.50 & 1.95 & 70.40 & 92.20 & 98.60 & 96.90 & 96.40 & 98.35 \\
\hline \multicolumn{13}{|l|}{$t_{b}^{*}$} \\
\hline 20 & 75.20 & 67.85 & 78.60 & 83.45 & 87.05 & 89.15 & 75.60 & 65.65 & 78.25 & 82.15 & 87.10 & 89.30 \\
\hline 30 & 47.25 & 37.45 & 49.50 & 56.70 & 67.90 & 72.55 & 46.75 & 34.95 & 47.75 & 54.35 & 64.30 & 68.45 \\
\hline 50 & 18.00 & 13.50 & 20.85 & 25.50 & 30.90 & 42.60 & 14.70 & 12.40 & 18.35 & 20.80 & 28.55 & 36.20 \\
\hline 70 & 10.80 & 9.30 & 12.30 & 14.90 & 19.80 & 27.95 & 8.25 & 8.00 & 9.20 & 12.85 & 17.25 & 23.40 \\
\hline 100 & 7.60 & 7.75 & 9.20 & 10.40 & 12.65 & 18.45 & 5.95 & 5.50 & 6.15 & 8.90 & 11.85 & 16.10 \\
\hline 200 & 6.20 & 6.35 & 5.95 & 6.30 & 7.30 & 8.45 & 3.10 & 2.55 & 3.25 & 5.40 & 8.20 & 10.15 \\
\hline
\end{tabular}

Notes: See notes to Table 11. The data generating process is the same as the one for Table 11, except $\rho_{i \varepsilon} \sim i i d U[0.2,0.4]$. 
Table 13: Size and Power of Panel Unit Root Tests with Two Factors $(m=2)$, Negatively Serially Correlated $\varepsilon_{i t}$ and Positively Serially Correlated $\Delta v_{i x t}$, Intercept and Linear Trend Case

\begin{tabular}{|c|c|c|c|c|c|c|c|c|c|c|c|c|}
\hline & \multicolumn{6}{|c|}{ Size: $\rho_{i}=\rho=1$} & \multicolumn{6}{|c|}{ Power: $\rho_{i} \sim \operatorname{iid} U[0.90,0.99]$} \\
\hline$(\mathrm{T}, \mathrm{N})$ & 20 & 30 & 50 & 70 & 100 & 200 & 20 & 30 & 50 & 70 & 100 & 200 \\
\hline $\operatorname{IPS}(p)$ & \multicolumn{6}{|c|}{$p=1$} & \multicolumn{6}{|c|}{$p=1$} \\
\hline 20 & 30.40 & 33.60 & 39.00 & 38.90 & 41.90 & 44.30 & 31.75 & 34.60 & 41.45 & 40.00 & 44.75 & 45.45 \\
\hline 30 & 31.25 & 35.10 & 38.95 & 38.55 & 40.85 & 44.95 & 32.40 & 36.65 & 41.00 & 40.85 & 43.40 & 47.80 \\
\hline 50 & 31.10 & 35.65 & 35.90 & 40.25 & 41.35 & 45.85 & 36.40 & 40.50 & 42.20 & 47.05 & 48.85 & 53.35 \\
\hline 70 & 32.15 & 35.10 & 38.40 & 40.90 & 42.70 & 45.30 & 40.45 & 46.65 & 51.10 & 52.80 & 54.05 & 57.10 \\
\hline 100 & 31.10 & 36.85 & 39.05 & 39.60 & 43.25 & 44.20 & 48.70 & 55.75 & 59.85 & 61.90 & 64.10 & 66.45 \\
\hline 200 & 30.10 & 34.80 & 36.30 & 39.45 & 42.40 & 43.25 & 72.50 & 81.55 & 84.75 & 86.25 & 88.60 & 90.20 \\
\hline $\operatorname{CIPS}(p)$ & \multicolumn{6}{|c|}{$p=1$} & \multicolumn{6}{|c|}{$p=1$} \\
\hline 20 & 5.55 & 6.50 & 5.65 & 6.30 & 6.05 & 6.80 & 4.35 & 4.80 & 4.25 & 4.85 & 4.30 & 4.00 \\
\hline 30 & 4.95 & 5.15 & 5.50 & 6.40 & 5.90 & 6.20 & 3.85 & 3.50 & 3.55 & 3.90 & 2.75 & 3.30 \\
\hline 50 & 4.80 & 4.25 & 5.20 & 6.60 & 4.70 & 6.25 & 4.10 & 4.00 & 4.15 & 3.75 & 2.70 & 2.65 \\
\hline 70 & 4.70 & 5.00 & 6.40 & 4.95 & 5.30 & 5.95 & 6.45 & 9.90 & 9.70 & 8.80 & 7.75 & 6.90 \\
\hline 100 & 5.00 & 4.80 & 4.75 & 5.30 & 5.35 & 4.70 & 17.60 & 27.90 & 37.10 & 39.65 & 45.00 & 60.05 \\
\hline 200 & 5.20 & 4.75 & 5.05 & 5.30 & 4.70 & 5.15 & 94.90 & 99.65 & 100.00 & 100.00 & 100.00 & 100.00 \\
\hline \multicolumn{13}{|l|}{$P_{b}$} \\
\hline 20 & 97.10 & 99.65 & 99.95 & 100.00 & 99.95 & 100.00 & 95.70 & 99.15 & 99.85 & 99.95 & 99.90 & 100.00 \\
\hline 30 & 94.55 & 99.30 & 99.95 & 100.00 & 100.00 & 100.00 & 91.25 & 97.55 & 99.65 & 99.75 & 99.35 & 99.75 \\
\hline 50 & 83.55 & 95.25 & 99.40 & 99.90 & 100.00 & 100.00 & 75.25 & 88.70 & 94.25 & 93.75 & 93.85 & 95.15 \\
\hline 70 & 72.40 & 85.45 & 96.70 & 99.10 & 99.90 & 100.00 & 64.50 & 78.85 & 89.50 & 87.65 & 88.10 & 89.65 \\
\hline 100 & 55.65 & 70.65 & 87.05 & 94.00 & 97.75 & 100.00 & 54.50 & 71.15 & 81.70 & 78.80 & 79.15 & 83.30 \\
\hline 200 & 27.95 & 35.95 & 47.95 & 59.50 & 68.00 & 93.05 & 36.10 & 51.50 & 68.40 & 67.50 & 68.30 & 74.15 \\
\hline \multicolumn{13}{|l|}{$P M S B$} \\
\hline 20 & 0.15 & 0.20 & 0.35 & 0.70 & 0.45 & 0.65 & 0.05 & 0.30 & 0.30 & 0.35 & 0.25 & 0.65 \\
\hline 30 & 1.15 & 1.65 & 1.40 & 2.60 & 2.55 & 3.55 & 1.50 & 1.85 & 3.70 & 3.75 & 3.45 & 9.30 \\
\hline 50 & 2.85 & 4.30 & 4.50 & 5.10 & 6.55 & 9.05 & 3.20 & 7.65 & 15.70 & 13.90 & 19.80 & 34.90 \\
\hline 70 & 2.60 & 5.30 & 6.65 & 8.05 & 7.10 & 11.55 & 6.60 & 15.55 & 30.45 & 30.00 & 35.40 & 45.70 \\
\hline 100 & 4.40 & 4.80 & 6.05 & 6.90 & 7.50 & 11.45 & 12.00 & 29.40 & 49.45 & 46.95 & 46.10 & 55.45 \\
\hline 200 & 4.10 & 5.00 & 5.45 & 5.30 & 7.45 & 9.05 & 33.30 & 60.10 & 79.30 & 70.85 & 70.55 & 75.40 \\
\hline \multicolumn{13}{|l|}{$t_{b}^{*}$} \\
\hline 20 & 99.25 & 99.75 & 99.95 & 100.00 & 99.95 & 99.95 & 99.25 & 99.80 & 100.00 & 99.95 & 99.90 & 100.00 \\
\hline 30 & 97.85 & 99.70 & 99.80 & 99.80 & 99.95 & 99.95 & 96.80 & 98.75 & 99.35 & 99.60 & 99.40 & 99.75 \\
\hline 50 & 86.85 & 94.85 & 98.30 & 99.20 & 99.75 & 99.95 & 81.65 & 90.20 & 94.75 & 94.30 & 94.50 & 95.75 \\
\hline 70 & 74.80 & 84.85 & 94.45 & 96.70 & 98.10 & 99.40 & 69.75 & 79.95 & 89.75 & 88.10 & 89.45 & 90.80 \\
\hline 100 & 53.95 & 68.15 & 84.20 & 89.70 & 94.05 & 98.35 & 56.25 & 72.10 & 82.70 & 80.70 & 80.70 & 84.75 \\
\hline 200 & 24.45 & 32.90 & 44.05 & 53.80 & 62.65 & 85.40 & 34.40 & 51.85 & 69.85 & 68.30 & 68.10 & 75.25 \\
\hline
\end{tabular}

Notes: See notes to Table 11. The data generating process is the same as the one for Table 11, except $\rho_{i \varepsilon} \sim i i d U[-0.2,-0.4]$. 
Table 14: Size of Panel Unit Root Tests for $u_{i t}$, Serially Correlated $f_{t}$ and $\Delta v_{i x t}$, Intercept and Linear Trend Case

\begin{tabular}{|c|c|c|c|c|c|c|c|c|c|c|c|c|}
\hline$(\mathrm{T}, \mathrm{N})$ & 20 & 30 & 50 & 70 & 100 & 200 & 20 & 30 & 50 & 70 & 100 & 200 \\
\hline $\operatorname{IPS}(p)$ & \multicolumn{6}{|c|}{$p=0$} & \multicolumn{6}{|c|}{$p=1$} \\
\hline 20 & 13.45 & 15.15 & 17.05 & 17.75 & 20.25 & 19.90 & 31.50 & 33.90 & 40.50 & 37.80 & 43.00 & 44.00 \\
\hline 30 & 12.45 & 12.80 & 14.00 & 14.85 & 17.60 & 18.40 & 31.50 & 35.15 & 38.75 & 38.55 & 41.60 & 44.85 \\
\hline 50 & 10.90 & 11.30 & 12.20 & 13.85 & 15.60 & 17.60 & 31.40 & 35.45 & 37.25 & 39.90 & 42.10 & 45.85 \\
\hline 70 & 9.40 & 11.20 & 13.55 & 14.80 & 14.90 & 16.90 & 31.35 & 34.00 & 38.70 & 41.80 & 42.05 & 44.90 \\
\hline 100 & 9.20 & 10.60 & 11.65 & 13.45 & 14.15 & 15.10 & 31.60 & 36.45 & 38.65 & 39.70 & 43.00 & 44.25 \\
\hline 200 & 8.95 & 9.95 & 10.30 & 12.80 & 13.05 & 15.15 & 29.95 & 33.95 & 36.65 & 39.10 & 41.35 & 42.95 \\
\hline $\operatorname{CIPS}(p)$ & \multicolumn{6}{|c|}{$p=0$} & \multicolumn{6}{|c|}{$p=1$} \\
\hline 20 & 12.55 & 14.35 & 17.85 & 18.90 & 20.65 & 24.70 & 10.00 & 11.80 & 10.05 & 12.70 & 12.55 & 13.60 \\
\hline 30 & 11.60 & 11.65 & 14.50 & 16.10 & 18.55 & 18.00 & 8.60 & 9.30 & 11.10 & 10.80 & 11.70 & 12.70 \\
\hline 50 & 8.90 & 9.55 & 10.70 & 12.60 & 12.45 & 16.10 & 7.25 & 7.95 & 9.50 & 10.85 & 8.45 & 11.45 \\
\hline 70 & 8.25 & 9.25 & 10.85 & 9.90 & 11.35 & 10.50 & 6.65 & 7.60 & 9.60 & 7.65 & 9.25 & 8.85 \\
\hline 100 & 7.45 & 7.75 & 7.40 & 8.00 & 8.80 & 9.10 & 7.00 & 6.95 & 6.70 & 7.50 & 7.40 & 7.45 \\
\hline 200 & 5.85 & 5.85 & 6.75 & 7.05 & 6.35 & 7.15 & 6.15 & 5.70 & 6.25 & 6.20 & 5.90 & 6.50 \\
\hline \multicolumn{13}{|l|}{$P_{b}$} \\
\hline 20 & - & - & - & - & - & - & 75.10 & 84.65 & 92.65 & 96.45 & 98.25 & 99.95 \\
\hline 30 & - & - & - & - & - & - & 55.90 & 69.60 & 83.30 & 91.60 & 95.65 & 99.35 \\
\hline 50 & - & - & - & - & - & - & 34.85 & 43.10 & 59.05 & 68.80 & 79.90 & 94.80 \\
\hline 70 & - & - & - & - & - & - & 25.30 & 32.05 & 41.50 & 52.40 & 62.00 & 83.95 \\
\hline 100 & - & - & - & - & - & - & 18.80 & 21.80 & 27.70 & 32.55 & 41.75 & 61.85 \\
\hline 200 & - & - & - & - & - & - & 10.70 & 12.25 & 13.40 & 14.50 & 18.60 & 26.95 \\
\hline \multicolumn{13}{|l|}{$P M S B$} \\
\hline 20 & - & - & - & - & - & - & 0.15 & 0.60 & 0.85 & 1.10 & 1.60 & 3.20 \\
\hline 30 & - & - & - & - & - & - & 0.85 & 0.70 & 0.80 & 1.25 & 2.20 & 2.25 \\
\hline 50 & - & - & - & - & - & - & 1.25 & 1.50 & 1.50 & 1.60 & 1.90 & 1.85 \\
\hline 70 & - & - & - & - & - & - & 1.10 & 2.05 & 2.25 & 2.05 & 1.70 & 2.45 \\
\hline 100 & - & - & - & - & - & - & 2.55 & 2.20 & 2.50 & 2.30 & 2.75 & 2.40 \\
\hline 200 & - & - & - & - & - & - & 2.65 & 3.55 & 3.10 & 2.90 & 3.50 & 2.90 \\
\hline \multicolumn{13}{|l|}{$t_{b}^{*}$} \\
\hline 20 & - & - & - & - & - & - & 91.35 & 94.90 & 97.80 & 99.05 & 99.15 & 99.60 \\
\hline 30 & - & - & - & - & - & - & 71.40 & 81.65 & 91.15 & 95.00 & 98.05 & 99.40 \\
\hline 50 & - & - & - & - & - & - & 40.70 & 49.65 & 66.65 & 75.95 & 84.95 & 94.65 \\
\hline 70 & - & - & - & - & - & - & 28.35 & 35.00 & 45.35 & 56.90 & 67.85 & 86.10 \\
\hline 100 & - & - & - & - & - & - & 19.10 & 22.35 & 30.75 & 36.15 & 46.95 & 65.10 \\
\hline 200 & - & - & - & - & - & - & 10.30 & 12.05 & 13.95 & 16.75 & 20.00 & 29.70 \\
\hline
\end{tabular}

Notes: See notes to Table 11. The data generating process is the same as the one for Table 11, except $\rho_{i}=\rho=1$ and $\rho_{f 1}=\rho_{f 2}=0.3$. The $P_{b}, P M S B$, and $t_{b}^{*}$ tests adopt automatic lag-order selection for the estimation of long-run variances, which explains the empty boxes in the above table. 
Table 15: Power of Panel Unit Root Tests, Serially Correlated $f_{t}$ and $\Delta v_{i x t}$, Intercept and Linear Trend Case

\begin{tabular}{|c|c|c|c|c|c|c|c|c|c|c|c|c|}
\hline$(\mathrm{T}, \mathrm{N})$ & 20 & 30 & 50 & 70 & 100 & 200 & 20 & 30 & 50 & 70 & 100 & 200 \\
\hline $\operatorname{IPS}(p)$ & \multicolumn{6}{|c|}{$p=0$} & \multicolumn{6}{|c|}{$p=1$} \\
\hline 20 & 14.00 & 16.20 & 17.35 & 18.65 & 20.30 & 20.45 & 31.65 & 35.45 & 41.10 & 39.75 & 45.10 & 46.10 \\
\hline 30 & 12.40 & 13.35 & 16.05 & 15.95 & 17.75 & 19.35 & 32.25 & 37.80 & 41.10 & 41.05 & 44.10 & 47.70 \\
\hline 50 & 10.80 & 13.90 & 14.90 & 18.10 & 17.70 & 21.50 & 36.00 & 39.45 & 42.80 & 47.40 & 48.90 & 53.35 \\
\hline 70 & 12.20 & 15.00 & 18.35 & 18.75 & 20.95 & 21.15 & 39.95 & 45.55 & 50.65 & 51.40 & 53.45 & 56.45 \\
\hline 100 & 13.05 & 17.35 & 20.50 & 23.65 & 23.60 & 27.20 & 47.35 & 54.05 & 58.45 & 60.70 & 63.10 & 65.10 \\
\hline 200 & 27.00 & 35.00 & 40.90 & 43.70 & 47.95 & 51.45 & 70.70 & 79.55 & 83.85 & 84.95 & 87.40 & 89.05 \\
\hline $\operatorname{CIPS}(p)$ & \multicolumn{6}{|c|}{$p=0$} & \multicolumn{6}{|c|}{$p=1$} \\
\hline 20 & 10.90 & 11.15 & 13.55 & 14.95 & 15.40 & 17.75 & 8.10 & 9.35 & 8.60 & 10.25 & 9.15 & 9.75 \\
\hline 30 & 9.65 & 9.30 & 11.05 & 12.35 & 11.00 & 10.50 & 7.00 & 7.45 & 6.85 & 8.45 & 6.75 & 7.70 \\
\hline 50 & 8.70 & 7.95 & 8.70 & 10.15 & 9.15 & 11.10 & 6.65 & 6.65 & 6.45 & 6.45 & 5.55 & 5.40 \\
\hline 70 & 10.25 & 15.50 & 17.35 & 18.30 & 17.45 & 19.10 & 7.75 & 11.20 & 11.95 & 11.70 & 10.50 & 10.40 \\
\hline 100 & 24.20 & 38.00 & 51.10 & 54.90 & 61.30 & 78.50 & 19.70 & 28.70 & 37.80 & 40.20 & 44.55 & 60.20 \\
\hline 200 & 96.40 & 99.85 & 100.00 & 100.00 & 100.00 & 100.00 & 94.30 & 99.50 & 100.00 & 100.00 & 100.00 & 100.00 \\
\hline \multicolumn{13}{|l|}{$P_{b}$} \\
\hline 20 & - & - & - & - & - & - & 71.80 & 82.10 & 91.50 & 93.90 & 96.55 & 98.10 \\
\hline 30 & - & - & - & - & - & - & 54.15 & 67.00 & 80.80 & 86.05 & 87.85 & 91.30 \\
\hline 50 & - & - & - & - & - & - & 33.80 & 46.10 & 59.65 & 63.55 & 68.65 & 76.40 \\
\hline 70 & - & - & - & - & - & - & 30.20 & 36.40 & 47.85 & 54.15 & 58.35 & 68.20 \\
\hline 100 & - & - & - & - & - & - & 25.35 & 29.40 & 39.00 & 42.35 & 47.25 & 56.65 \\
\hline 200 & - & - & - & - & - & - & 18.80 & 22.05 & 25.50 & 32.75 & 37.95 & 46.25 \\
\hline \multicolumn{13}{|l|}{$P M S B$} \\
\hline 20 & - & - & - & - & - & - & 0.20 & 0.30 & 0.55 & 0.70 & 1.25 & 2.00 \\
\hline 30 & - & - & - & - & - & - & 0.50 & 0.65 & 0.75 & 0.80 & 0.80 & 1.20 \\
\hline 50 & - & - & - & - & - & - & 1.15 & 2.70 & 4.55 & 3.75 & 5.55 & 11.20 \\
\hline 70 & - & - & - & - & - & - & 3.00 & 8.00 & 15.50 & 15.55 & 19.90 & 31.15 \\
\hline 100 & - & - & - & - & - & - & 8.25 & 20.20 & 38.95 & 37.25 & 37.75 & 48.50 \\
\hline 200 & - & - & - & - & - & - & 30.35 & 56.50 & 76.15 & 69.20 & 68.00 & 73.75 \\
\hline \multicolumn{13}{|l|}{$t_{b}^{*}$} \\
\hline 20 & - & - & - & - & - & - & 90.55 & 93.95 & 97.20 & 98.20 & 98.65 & 99.20 \\
\hline 30 & - & - & - & - & - & - & 72.65 & 79.45 & 89.75 & 91.50 & 92.70 & 95.10 \\
\hline 50 & - & - & - & - & - & - & 42.85 & 52.95 & 67.20 & 71.60 & 73.90 & 80.10 \\
\hline 70 & - & - & - & - & - & - & 34.30 & 39.35 & 53.65 & 58.15 & 63.60 & 72.15 \\
\hline 100 & - & - & - & - & - & - & 26.35 & 31.20 & 41.10 & 45.50 & 50.30 & 59.80 \\
\hline 200 & - & - & - & - & - & - & 18.60 & 21.75 & 26.50 & 32.05 & 37.80 & 47.00 \\
\hline
\end{tabular}

Notes: See notes to Table 11. The data generating process is the same as the one for Table 11, except $\rho_{i} \sim i i d U[0.90,0.99]$ and $\rho_{f 1}=\rho_{f 2}=0.3$. The $P_{b}, P M S B$, and $t_{b}^{*}$ tests adopt automatic lag-order selection for the estimation of long-run variances, which explains the empty boxes in the above table. 
Table 16: Size of Panel Unit Root Tests, Supposing Three Factors Exist when There are Actually Two Factors $(m=2)$, Serially Correlated $\Delta v_{i x t}$, Intercept and Linear Trend Case

\begin{tabular}{|c|c|c|c|c|c|c|c|c|c|c|c|c|}
\hline$(\mathrm{T}, \mathrm{N})$ & 20 & 30 & 50 & 70 & 100 & 200 & 20 & 30 & 50 & 70 & 100 & 200 \\
\hline $\operatorname{IPS}(p)$ & \multicolumn{6}{|c|}{$p=0$} & \multicolumn{6}{|c|}{$p=1$} \\
\hline 20 & 30.80 & 34.10 & 37.65 & 39.00 & 42.40 & 43.55 & 30.75 & 35.20 & 37.85 & 38.55 & 43.40 & 43.50 \\
\hline 30 & 30.60 & 34.70 & 36.85 & 40.40 & 42.15 & 45.25 & 31.00 & 35.25 & 36.85 & 41.65 & 41.90 & 46.30 \\
\hline 50 & 33.80 & 34.05 & 41.75 & 41.60 & 41.80 & 45.45 & 33.10 & 33.50 & 41.85 & 41.55 & 42.90 & 45.15 \\
\hline 70 & 33.55 & 34.35 & 41.40 & 41.65 & 43.45 & 46.00 & 33.60 & 35.25 & 40.90 & 41.30 & 43.15 & 46.00 \\
\hline 100 & 31.00 & 35.05 & 38.10 & 40.50 & 41.85 & 46.25 & 31.10 & 34.05 & 38.95 & 40.70 & 41.55 & 45.70 \\
\hline 200 & 33.20 & 33.65 & 39.55 & 40.55 & 42.65 & 46.60 & 33.75 & 34.60 & 39.30 & 40.95 & 42.90 & 46.15 \\
\hline $\operatorname{CIPS}(p)$ & \multicolumn{6}{|c|}{$p=0$} & \multicolumn{6}{|c|}{$p=1$} \\
\hline 20 & 8.70 & 7.80 & 9.35 & 10.45 & 9.90 & 10.60 & 7.15 & 6.85 & 6.65 & 7.05 & 8.10 & 7.30 \\
\hline 30 & 7.45 & 6.95 & 8.50 & 10.40 & 9.90 & 10.15 & 6.95 & 6.55 & 6.35 & 8.15 & 8.05 & 8.45 \\
\hline 50 & 7.70 & 6.90 & 6.50 & 7.40 & 8.30 & 8.85 & 6.85 & 7.35 & 6.00 & 6.25 & 7.40 & 7.20 \\
\hline 70 & 5.50 & 6.65 & 7.15 & 7.05 & 7.75 & 7.65 & 4.40 & 6.70 & 6.70 & 6.70 & 6.75 & 6.70 \\
\hline 100 & 6.40 & 5.65 & 6.85 & 6.25 & 7.15 & 6.20 & 6.00 & 5.40 & 6.25 & 5.70 & 6.60 & 6.10 \\
\hline 200 & 5.65 & 6.15 & 6.00 & 5.10 & 5.40 & 7.40 & 5.45 & 6.45 & 5.65 & 4.90 & 5.40 & 7.15 \\
\hline \multicolumn{13}{|l|}{$P_{b}$} \\
\hline 20 & - & - & - & - & - & - & 69.40 & 77.60 & 90.70 & 95.45 & 98.20 & 99.85 \\
\hline 30 & - & - & - & - & - & - & 53.50 & 62.25 & 79.15 & 87.40 & 94.20 & 99.50 \\
\hline 50 & - & - & - & - & - & - & 34.00 & 41.50 & 54.20 & 64.45 & 77.90 & 93.85 \\
\hline 70 & - & - & - & - & - & - & 27.10 & 31.45 & 40.80 & 47.60 & 60.60 & 82.20 \\
\hline 100 & - & - & - & - & - & - & 18.60 & 20.65 & 28.15 & 32.65 & 39.05 & 59.40 \\
\hline 200 & - & - & - & - & - & - & 11.35 & 12.35 & 14.05 & 15.30 & 19.45 & 27.80 \\
\hline \multicolumn{13}{|l|}{$P M S B$} \\
\hline 20 & - & - & - & - & - & - & 0.10 & 0.40 & 0.35 & 0.25 & 0.55 & 0.80 \\
\hline 30 & - & - & - & - & - & - & 0.80 & 0.75 & 0.70 & 0.60 & 0.65 & 0.50 \\
\hline 50 & - & - & - & - & - & - & 1.55 & 1.65 & 1.00 & 0.90 & 0.75 & 0.60 \\
\hline 70 & - & - & - & - & - & - & 1.80 & 2.00 & 1.70 & 0.90 & 1.00 & 0.85 \\
\hline 100 & - & - & - & - & - & - & 2.40 & 2.40 & 2.35 & 1.65 & 1.85 & 1.20 \\
\hline 200 & - & - & - & - & - & - & 3.35 & 3.40 & 3.20 & 3.70 & 2.70 & 2.00 \\
\hline \multicolumn{13}{|l|}{$t_{b}^{*}$} \\
\hline 20 & - & - & - & - & - & - & 98.85 & 99.65 & 99.75 & 99.95 & 100.00 & 100.00 \\
\hline 30 & - & - & - & - & - & - & 94.45 & 97.90 & 99.75 & 99.55 & 99.90 & 100.00 \\
\hline 50 & - & - & - & - & - & - & 80.15 & 89.85 & 95.90 & 97.45 & 98.80 & 99.80 \\
\hline 70 & - & - & - & - & - & - & 67.70 & 79.20 & 91.00 & 95.05 & 97.70 & 99.25 \\
\hline 100 & - & - & - & - & - & - & 45.75 & 63.90 & 81.80 & 88.80 & 93.35 & 98.35 \\
\hline 200 & - & - & - & - & - & - & 24.50 & 35.75 & 53.85 & 67.20 & 79.85 & 91.80 \\
\hline
\end{tabular}

Notes: See notes to Table 11. The data generating process is the same as the one for Table 11, except $\gamma_{i 2}=\gamma_{2}=0$ and $\gamma_{i x 1}=\gamma_{x 1}=0$. The CIPS test is based on cross section augmentation using $y_{i t}$ and $x_{i t}$. The $P$, PMSB, and $t_{b}^{*}$ tests are based on three extracted factors from the $y_{i t}$ series using principal components. These tests adopt automatic lag-order selection for the estimation of long-run variances, which explains the empty boxes in the above table. 
Table 17: Power of Panel Unit Root Tests, Supposing Three Factors Exist when There are Actually Two Factors $(m=2)$, Serially Correlated $\Delta v_{i x t}$, Intercept and Linear Trend Case

\begin{tabular}{|c|c|c|c|c|c|c|c|c|c|c|c|c|}
\hline$(\mathrm{T}, \mathrm{N})$ & 20 & 30 & 50 & 70 & 100 & 200 & 20 & 30 & 50 & 70 & 100 & 200 \\
\hline $\operatorname{IPS}(p)$ & \multicolumn{6}{|c|}{$p=0$} & \multicolumn{6}{|c|}{$p=1$} \\
\hline 20 & 32.40 & 35.40 & 39.25 & 40.00 & 43.10 & 44.85 & 31.45 & 36.85 & 40.00 & 40.55 & 44.80 & 45.95 \\
\hline 30 & 33.75 & 37.65 & 40.20 & 44.05 & 45.55 & 49.10 & 32.25 & 37.70 & 40.35 & 45.35 & 44.85 & 48.80 \\
\hline 50 & 39.30 & 42.65 & 48.75 & 49.20 & 51.50 & 54.20 & 38.60 & 42.00 & 47.75 & 49.35 & 50.55 & 54.60 \\
\hline 70 & 44.10 & 48.75 & 55.00 & 57.45 & 58.90 & 61.85 & 43.80 & 47.60 & 54.10 & 55.20 & 57.50 & 61.55 \\
\hline 100 & 50.55 & 59.20 & 61.70 & 65.30 & 66.20 & 73.05 & 50.00 & 57.45 & 60.50 & 64.15 & 65.60 & 71.75 \\
\hline 200 & 79.70 & 85.55 & 90.60 & 88.50 & 92.30 & 94.00 & 77.90 & 84.00 & 88.90 & 87.80 & 91.00 & 93.10 \\
\hline $\operatorname{CIPS}(p)$ & \multicolumn{6}{|c|}{$p=0$} & \multicolumn{6}{|c|}{$p=1$} \\
\hline 20 & 8.70 & 7.75 & 8.20 & 10.00 & 8.65 & 9.65 & 6.45 & 7.45 & 6.25 & 7.40 & 7.00 & 6.35 \\
\hline 30 & 7.60 & 7.40 & 8.10 & 10.60 & 9.20 & 8.45 & 7.10 & 6.55 & 6.25 & 7.80 & 6.95 & 6.30 \\
\hline 50 & 8.65 & 8.60 & 8.75 & 11.20 & 9.95 & 9.85 & 7.05 & 7.05 & 7.05 & 8.70 & 7.85 & 6.45 \\
\hline 70 & 9.65 & 12.30 & 15.95 & 17.30 & 18.20 & 18.75 & 6.85 & 11.05 & 11.70 & 13.55 & 12.80 & 12.90 \\
\hline 100 & 19.65 & 23.35 & 37.40 & 43.90 & 44.90 & 55.25 & 15.90 & 18.65 & 29.55 & 33.20 & 33.00 & 40.75 \\
\hline 200 & 84.70 & 98.00 & 100.00 & 99.95 & 100.00 & 100.00 & 77.60 & 95.45 & 99.75 & 99.95 & 100.00 & 100.00 \\
\hline \multicolumn{13}{|l|}{$P_{b}$} \\
\hline 20 & - & - & - & - & - & - & 69.50 & 78.90 & 91.00 & 95.40 & 98.45 & 99.90 \\
\hline 30 & - & - & - & - & - & - & 53.60 & 64.15 & 80.15 & 88.30 & 94.20 & 99.25 \\
\hline 50 & - & - & - & - & - & - & 35.60 & 46.25 & 60.95 & 69.95 & 82.85 & 96.15 \\
\hline 70 & - & - & - & - & - & - & 28.60 & 35.50 & 49.90 & 59.15 & 71.55 & 90.65 \\
\hline 100 & - & - & - & - & - & - & 21.70 & 26.40 & 38.55 & 46.15 & 58.75 & 86.10 \\
\hline 200 & - & - & - & - & - & - & 12.55 & 17.65 & 27.55 & 33.40 & 47.00 & 77.65 \\
\hline \multicolumn{13}{|l|}{$P M S B$} \\
\hline 20 & - & - & - & - & - & - & 0.15 & 0.30 & 0.30 & 0.95 & 0.55 & 3.00 \\
\hline 30 & - & - & - & - & - & - & 0.70 & 0.50 & 1.00 & 0.90 & 1.75 & 5.60 \\
\hline 50 & - & - & - & - & - & - & 2.90 & 4.10 & 7.25 & 8.55 & 14.85 & 35.25 \\
\hline 70 & - & - & - & - & - & - & 8.35 & 14.95 & 24.80 & 32.70 & 48.45 & 81.30 \\
\hline 100 & - & - & - & - & - & - & 18.85 & 35.10 & 57.90 & 67.95 & 83.45 & 97.90 \\
\hline 200 & - & - & - & - & - & - & 68.70 & 86.30 & 94.70 & 97.75 & 99.70 & 100.00 \\
\hline \multicolumn{13}{|l|}{$t_{b}^{*}$} \\
\hline 20 & - & - & - & - & - & - & 98.90 & 99.75 & 99.85 & 100.00 & 100.00 & 100.00 \\
\hline 30 & - & - & - & - & - & - & 95.60 & 98.60 & 99.70 & 99.90 & 99.95 & 100.00 \\
\hline 50 & - & - & - & - & - & - & 81.95 & 91.65 & 97.75 & 99.25 & 99.90 & 99.95 \\
\hline 70 & - & - & - & - & - & - & 66.75 & 83.45 & 95.20 & 98.60 & 99.60 & 100.00 \\
\hline 100 & - & - & - & - & - & - & 47.85 & 63.80 & 87.75 & 95.20 & 98.40 & 99.90 \\
\hline 200 & - & - & - & - & - & - & 19.25 & 31.35 & 58.90 & 74.75 & 87.00 & 97.15 \\
\hline
\end{tabular}

Notes: See notes to Table 11. The data generating process is the same as the one for Table 11, except $\gamma_{i 2}=\gamma_{2}=0$ and $\gamma_{i x 1}=\gamma_{x 1}=0$. The CIPS test is based on cross section augmentation using $y_{i t}$ and $x_{i t}$. The $P_{b}, P M S B$, and $t_{b}^{*}$ tests are based on three extracted factors from the $y_{i t}$ series using principal components. These tests adopt automatic lag-order selection for the estimation of long-run variances, which explains the empty boxes in the above table. 
Table 18: Size of Panel Unit Root Tests, Supposing Three Factors Exist when There are Actually Two Factors $(m=2)$, Serially Correlated $\Delta v_{i x t}$, Intercept and Linear Trend Case. For the $P_{b}, P M S B$, and $t_{b}^{*}$ tests the Number of Factors is Estimated.

\begin{tabular}{|c|c|c|c|c|c|c|c|c|c|c|c|c|}
\hline$(\mathrm{T}, \mathrm{N})$ & 20 & 30 & 50 & 70 & 100 & 200 & 20 & 30 & 50 & 70 & 100 & 200 \\
\hline $\operatorname{IPS}(p)$ & \multicolumn{6}{|c|}{$p=0$} & \multicolumn{6}{|c|}{$p=1$} \\
\hline 20 & 30.80 & 34.10 & 37.65 & 39.00 & 42.40 & 43.55 & 30.75 & 35.20 & 37.85 & 38.55 & 43.40 & 43.50 \\
\hline 30 & 30.60 & 34.70 & 36.85 & 40.40 & 42.15 & 45.25 & 31.00 & 35.25 & 36.85 & 41.65 & 41.90 & 46.30 \\
\hline 50 & 33.80 & 34.05 & 41.75 & 41.60 & 41.80 & 45.45 & 33.10 & 33.50 & 41.85 & 41.55 & 42.90 & 45.15 \\
\hline 70 & 33.55 & 34.35 & 41.40 & 41.65 & 43.45 & 46.00 & 33.60 & 35.25 & 40.90 & 41.30 & 43.15 & 46.00 \\
\hline 100 & 31.00 & 35.05 & 38.10 & 40.50 & 41.85 & 46.25 & 31.10 & 34.05 & 38.95 & 40.70 & 41.55 & 45.70 \\
\hline 200 & 33.20 & 33.65 & 39.55 & 40.55 & 42.65 & 46.60 & 33.75 & 34.60 & 39.30 & 40.95 & 42.90 & 46.15 \\
\hline $\operatorname{CIPS}(p)$ & \multicolumn{6}{|c|}{$p=0$} & \multicolumn{6}{|c|}{$p=1$} \\
\hline 20 & 8.70 & 7.80 & 9.35 & 10.45 & 9.90 & 10.60 & 7.15 & 6.85 & 6.65 & 7.05 & 8.10 & 7.30 \\
\hline 30 & 7.45 & 6.95 & 8.50 & 10.40 & 9.90 & 10.15 & 6.95 & 6.55 & 6.35 & 8.15 & 8.05 & 8.45 \\
\hline 50 & 7.70 & 6.90 & 6.50 & 7.40 & 8.30 & 8.85 & 6.85 & 7.35 & 6.00 & 6.25 & 7.40 & 7.20 \\
\hline 70 & 5.50 & 6.65 & 7.15 & 7.05 & 7.75 & 7.65 & 4.40 & 6.70 & 6.70 & 6.70 & 6.75 & 6.70 \\
\hline 100 & 6.40 & 5.65 & 6.85 & 6.25 & 7.15 & 6.20 & 6.00 & 5.40 & 6.25 & 5.70 & 6.60 & 6.10 \\
\hline 200 & 5.65 & 6.15 & 6.00 & 5.10 & 5.40 & 7.40 & 5.45 & 6.45 & 5.65 & 4.90 & 5.40 & 7.15 \\
\hline \multicolumn{13}{|l|}{$P_{b}$} \\
\hline 20 & - & - & - & - & - & - & 68.60 & 78.20 & 88.50 & 91.55 & 96.20 & 96.95 \\
\hline 30 & - & - & - & - & - & - & 54.35 & 63.65 & 79.40 & 85.45 & 92.95 & 97.80 \\
\hline 50 & - & - & - & - & - & - & 35.85 & 42.35 & 55.90 & 64.40 & 76.15 & 94.80 \\
\hline 70 & - & - & - & - & - & - & 25.55 & 30.70 & 40.80 & 47.65 & 59.70 & 82.50 \\
\hline 100 & - & - & - & - & - & - & 19.55 & 21.60 & 29.40 & 32.65 & 40.05 & 59.55 \\
\hline 200 & - & - & - & - & - & - & 12.80 & 13.35 & 13.85 & 15.25 & 19.65 & 27.25 \\
\hline \multicolumn{13}{|l|}{$P M S B$} \\
\hline 20 & - & - & - & - & - & - & 0.10 & 0.30 & 0.55 & 0.55 & 0.70 & 0.90 \\
\hline 30 & - & - & - & - & - & - & 0.75 & 0.75 & 1.05 & 0.95 & 0.35 & 1.00 \\
\hline 50 & - & - & - & - & - & - & 2.00 & 1.90 & 1.50 & 1.05 & 0.80 & 0.35 \\
\hline 70 & - & - & - & - & - & - & 2.95 & 2.00 & 2.00 & 1.00 & 0.90 & 0.70 \\
\hline 100 & - & - & - & - & - & - & 2.35 & 3.10 & 1.95 & 1.65 & 1.70 & 1.30 \\
\hline 200 & - & - & - & - & - & - & 4.30 & 3.55 & 3.15 & 3.55 & 2.55 & 1.95 \\
\hline \multicolumn{13}{|l|}{$t_{b}^{*}$} \\
\hline 20 & - & - & - & - & - & - & 67.95 & 82.25 & 87.60 & 86.05 & 94.15 & 93.15 \\
\hline 30 & - & - & - & - & - & - & 49.45 & 70.65 & 82.05 & 83.30 & 92.85 & 95.40 \\
\hline 50 & - & - & - & - & - & - & 29.75 & 45.10 & 62.60 & 69.90 & 80.75 & 91.00 \\
\hline 70 & - & - & - & - & - & - & 20.75 & 32.60 & 43.95 & 53.65 & 64.50 & 81.50 \\
\hline 100 & - & - & - & - & - & - & 16.10 & 22.50 & 31.65 & 36.45 & 43.60 & 63.75 \\
\hline 200 & - & - & - & - & - & - & 11.50 & 11.75 & 13.35 & 15.25 & 19.65 & 28.85 \\
\hline
\end{tabular}

Notes: See notes to Table 11. The data generating process is the same as the one for Table 11 , except $\gamma_{i 2}=\gamma_{2}=0$ and $\gamma_{i x 1}=\gamma_{x 1}=0$. The CIPS test is based on cross section augmentation using $y_{i t}$ and $x_{i t}$. For the $P_{b}, P M S B$, and $t_{b}^{*}$ tests, the number of factors is determined by the BIC3 criterion of Bai and $\mathrm{Ng}(2002)$ with the maximum number set to three, and the factors are extracted from the $y_{i t}$ series using principal components. These tests adopt automatic lag-order selection for the estimation of long-run variances, which explains the empty boxes in the above table. 
Table 19: Power of Panel Unit Root Tests, Supposing Three Factors Exist when There are Actually Two Factors $(m=2)$, Serially Correlated $\Delta v_{i x t}$, Intercept and Linear Trend Case. For the $P_{b}, P M S B$, and $t_{b}^{*}$ tests the Number of Factors is Estimated.

\begin{tabular}{|c|c|c|c|c|c|c|c|c|c|c|c|c|}
\hline$(\mathrm{T}, \mathrm{N})$ & 20 & 30 & 50 & 70 & 100 & 200 & 20 & 30 & 50 & 70 & 100 & 200 \\
\hline $\operatorname{IPS}(p)$ & \multicolumn{6}{|c|}{$p=0$} & \multicolumn{6}{|c|}{$p=1$} \\
\hline 20 & 32.40 & 35.40 & 39.25 & 40.00 & 43.10 & 44.85 & 31.45 & 36.85 & 40.00 & 40.55 & 44.80 & 45.95 \\
\hline 30 & 33.75 & 37.65 & 40.20 & 44.05 & 45.55 & 49.10 & 32.25 & 37.70 & 40.35 & 45.35 & 44.85 & 48.80 \\
\hline 50 & 39.30 & 42.65 & 48.75 & 49.20 & 51.50 & 54.20 & 38.60 & 42.00 & 47.75 & 49.35 & 50.55 & 54.60 \\
\hline 70 & 44.10 & 48.75 & 55.00 & 57.45 & 58.90 & 61.85 & 43.80 & 47.60 & 54.10 & 55.20 & 57.50 & 61.55 \\
\hline 100 & 50.55 & 59.20 & 61.70 & 65.30 & 66.20 & 73.05 & 50.00 & 57.45 & 60.50 & 64.15 & 65.60 & 71.75 \\
\hline 200 & 79.70 & 85.55 & 90.60 & 88.50 & 92.30 & 94.00 & 77.90 & 84.00 & 88.90 & 87.80 & 91.00 & 93.10 \\
\hline $\operatorname{CIPS}(p)$ & \multicolumn{6}{|c|}{$p=0$} & \multicolumn{6}{|c|}{$p=1$} \\
\hline 20 & 8.70 & 7.75 & 8.20 & 10.00 & 8.65 & 9.65 & 6.45 & 7.45 & 6.25 & 7.40 & 7.00 & 6.35 \\
\hline 30 & 7.60 & 7.40 & 8.10 & 10.60 & 9.20 & 8.45 & 7.10 & 6.55 & 6.25 & 7.80 & 6.95 & 6.30 \\
\hline 50 & 8.65 & 8.60 & 8.75 & 11.20 & 9.95 & 9.85 & 7.05 & 7.05 & 7.05 & 8.70 & 7.85 & 6.45 \\
\hline 70 & 9.65 & 12.30 & 15.95 & 17.30 & 18.20 & 18.75 & 6.85 & 11.05 & 11.70 & 13.55 & 12.80 & 12.90 \\
\hline 100 & 19.65 & 23.35 & 37.40 & 43.90 & 44.90 & 55.25 & 15.90 & 18.65 & 29.55 & 33.20 & 33.00 & 40.75 \\
\hline 200 & 84.70 & 98.00 & 100.00 & 99.95 & 100.00 & 100.00 & 77.60 & 95.45 & 99.75 & 99.95 & 100.00 & 100.00 \\
\hline \multicolumn{13}{|l|}{$P_{b}$} \\
\hline 20 & - & - & - & - & - & - & 70.00 & 78.80 & 89.45 & 90.55 & 95.65 & 96.45 \\
\hline 30 & - & - & - & - & - & - & 55.65 & 64.35 & 79.30 & 84.30 & 91.35 & 94.20 \\
\hline 50 & - & - & - & - & - & - & 37.80 & 45.90 & 61.95 & 68.15 & 75.60 & 84.70 \\
\hline 70 & - & - & - & - & - & - & 29.25 & 37.45 & 49.50 & 56.00 & 65.60 & 75.55 \\
\hline 100 & - & - & - & - & - & - & 22.90 & 28.00 & 38.60 & 44.30 & 52.95 & 64.85 \\
\hline 200 & - & - & - & - & - & - & 12.70 & 18.50 & 27.75 & 32.30 & 39.40 & 51.20 \\
\hline \multicolumn{13}{|l|}{$P M S B$} \\
\hline 20 & - & - & - & - & - & - & 0.15 & 0.15 & 0.50 & 0.75 & 0.65 & 1.15 \\
\hline 30 & - & - & - & - & - & - & 1.15 & 1.25 & 1.90 & 1.90 & 1.10 & 2.55 \\
\hline 50 & - & - & - & - & - & - & 5.10 & 5.15 & 9.50 & 9.20 & 12.05 & 22.45 \\
\hline 70 & - & - & - & - & - & - & 11.00 & 17.20 & 26.75 & 27.20 & 39.55 & 52.70 \\
\hline 100 & - & - & - & - & - & - & 25.25 & 38.00 & 54.05 & 55.15 & 65.35 & 73.10 \\
\hline 200 & - & - & - & - & - & - & 72.50 & 84.20 & 87.35 & 81.95 & 91.60 & 92.20 \\
\hline \multicolumn{13}{|l|}{$t_{b}^{*}$} \\
\hline 20 & - & - & - & - & - & - & 67.95 & 82.05 & 87.85 & 86.05 & 94.10 & 93.65 \\
\hline 30 & - & - & - & - & - & - & 50.15 & 70.80 & 80.95 & 80.55 & 91.30 & 93.65 \\
\hline 50 & - & - & - & - & - & - & 31.00 & 47.55 & 63.05 & 66.80 & 77.35 & 83.55 \\
\hline 70 & - & - & - & - & - & - & 20.65 & 35.90 & 48.35 & 54.05 & 63.25 & 74.40 \\
\hline 100 & - & - & - & - & - & - & 17.00 & 24.30 & 33.85 & 38.10 & 46.15 & 60.10 \\
\hline 200 & - & - & - & - & - & - & 8.75 & 14.10 & 20.35 & 24.65 & 28.75 & 39.55 \\
\hline
\end{tabular}

Notes: See notes to Table 11. The data generating process is the same as the one for Table 11, except $\gamma_{i 2}=\gamma_{2}=0$ and $\gamma_{i x 1}=\gamma_{x 1}=0$. The CIPS test is based on cross section augmentation using $y_{i t}$ and $x_{i t}$. For the $P_{b}, P M S B$, and $t_{b}^{*}$ tests, the number of factors is determined by the BIC3 criterion of Bai and $\mathrm{Ng}(2002)$ with the maximum number set to three, and the factors are extracted from the $y_{i t}$ series using principal components. These tests adopt automatic lag-order selection for the estimation of long-run variances, which explains the empty boxes in the above table. 